\title{
Early experimental results of using a novel delivery carrier, Hyaluronan-phosphatidylethanolamine (HA-PE) which may allow simple bladder instillation of Botulinum Toxin A as effectively as direct detrusor muscle injection.
}

Mohamed G. El Shatoury, The University of Western Ontario

Supervisor: Sumit Dave, The University of Western Ontario

A thesis submitted in partial fulfillment of the requirements for the Master of Science degree in Surgery

(C) Mohamed G. El Shatoury 2016

Follow this and additional works at: https://ir.lib.uwo.ca/etd

Part of the Surgery Commons, and the Urology Commons

\section{Recommended Citation}

El Shatoury, Mohamed G., "Early experimental results of using a novel delivery carrier, Hyaluronanphosphatidylethanolamine (HA-PE) which may allow simple bladder instillation of Botulinum Toxin A as effectively as direct detrusor muscle injection." (2016). Electronic Thesis and Dissertation Repository. 4233.

https://ir.lib.uwo.ca/etd/4233

This Dissertation/Thesis is brought to you for free and open access by Scholarship@Western. It has been accepted for inclusion in Electronic Thesis and Dissertation Repository by an authorized administrator of Scholarship@Western. For more information, please contact wlswadmin@uwo.ca. 


\begin{abstract}
Intra-detrusor Botulinum Toxin-A (BTX-A) injection is the gold standard treatment for detrusor overactivity in clinical practice when medical treatment fails. Despite satisfactory results of BTX-A injection, the endoscopic procedure still has risks of anaesthetic and operative complications. Several studies have investigated more simple ways to deliver BTXA to the detrusor muscle but with limited success. In our study, we assessed the evidence of BTX-A delivery using a newly modulated hyaluronan-phosphatidylethanolamine (HA-PE) carrier in a rat model through BTX-A-HA-PE bladder instillations. We used histological evidence of SNAP-25 protein cleavage and detection of Alexa Fluor®594-labelled BTX-A as a proof of physical BTX-A delivery across the urothelium. In addition, we assessed the functional impact of using HA-PE on rat detrusor muscle through urodynamic assessment both at baseline and 2 weeks later after $1 \%$ acetic acid instillation. We found positive histological evidence of SNAP-25 cleavage, detection of Alexa Fluor®594-BTX-A red fluorescence in bladder tissue supporting that HA-PE may be an efficient carrier to deliver BTX-A across the urothelium.
\end{abstract}

Keywords: Botulinum Toxin A, Detrusor overactivity, modulated carrier, Bladder instillations, SNAP-25 cleavage, and urodynamic assessment. 


\section{Dedication}

I would like to dedicate this work to my beloved mother, my biggest fan and the everlasting inspiration of my success. To my beloved family, who inspires me to give my best in everything I do. To my sisters for supporting and encouraging me to do my best from the beginning to the end. Above all, I dedicate this work to God, for giving me the guidance and help to make this extraordinary accomplishment in my academic career. 


\section{Acknowledgments}

I'd like to thank everyone who contributed directly and indirectly for the fulfilment of this project. Special thanks go to my supervisor, Dr. Sumit Dave for his tremendous help and support, to my co-supervisors, Dr. Eva Turley and Dr. Arjang Yazdani for their generous support and cooperation. I'd like also to thank Dr. Abdelrahman Lawendy for his unconditional support and encouragement. I want to extend special thanks to Ling De Young for her support and mentoring during the experiment and Hon Leong for his help and cooperation in examining the slides. It is hard to go through this project without acknowledging the financial support from the department of Surgery at Western University through the internal grant funding that made this work possible. Special thanks to Kait Al for her extraordinary help and support in revision and editing of this work. I'm so grateful to everyone in the animal lab at Lawson Research institute for making my job easier and successful. 


\section{Table of Contents}

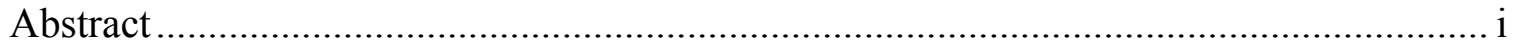

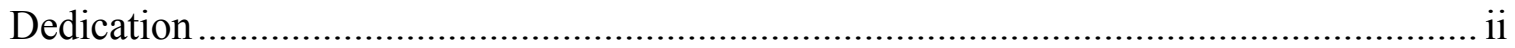

Acknowledgments...............................................................................................ii

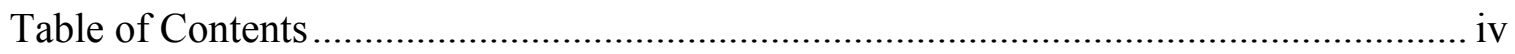

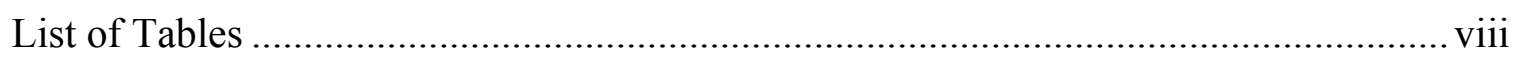

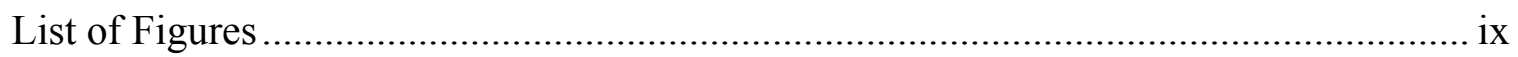

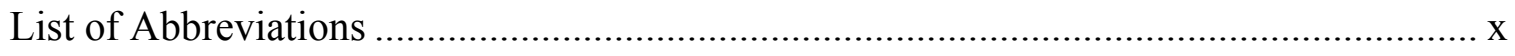

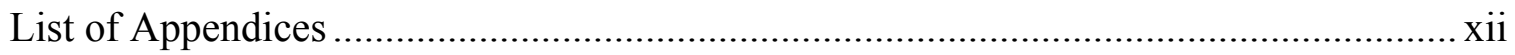

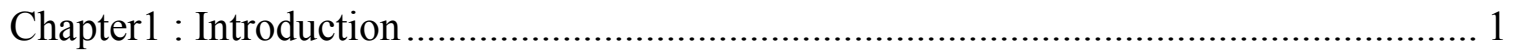

1.1 Bladder function and dysfunction ........................................................................ 1

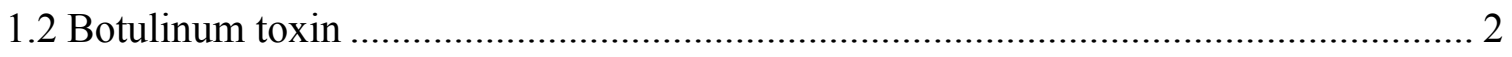

1.3 Hyaluronic acid-based topical delivery of proteins ...................................................... 4

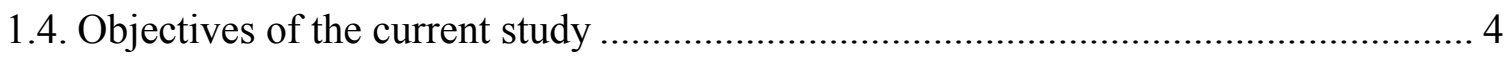

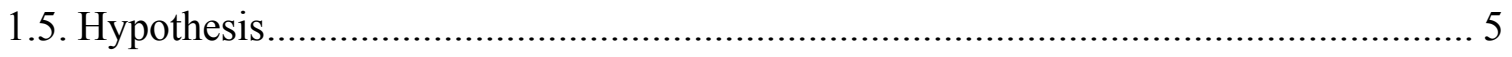

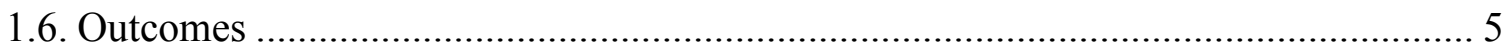

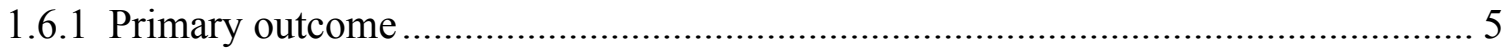

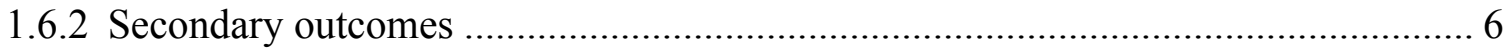

Chapter 2: Review of literature ................................................................................ 7

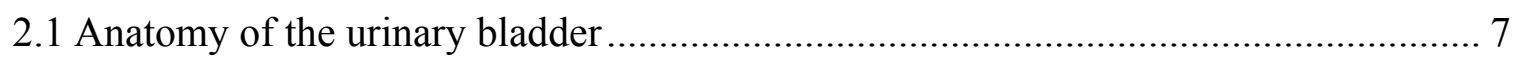

2.2 Architecture of the bladder wall.......................................................................... 10

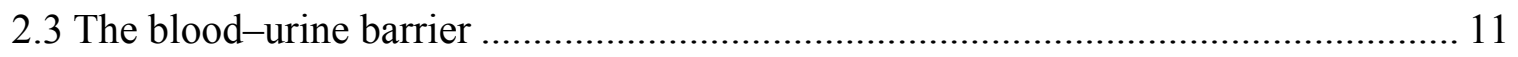

2.4. Physiological aspects of bladder function............................................................ 12

2.4.1 Muscarinic receptors of the urinary bladder ........................................................ 12

2.4.2 Adrenergic receptors of the urinary bladder ………........................................ 13

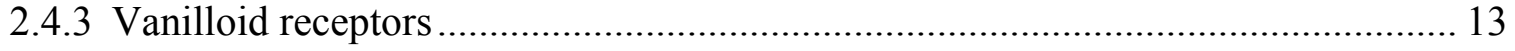


2.4.4 Neural circuits controlling bladder storage and elimination ............................... 14

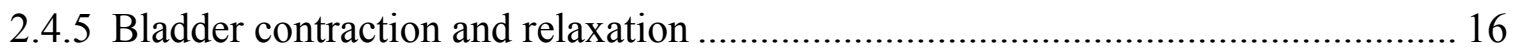

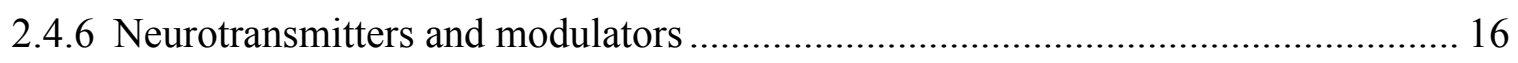

2.5. Current treatment for neurogenic bladder .................................................. 17

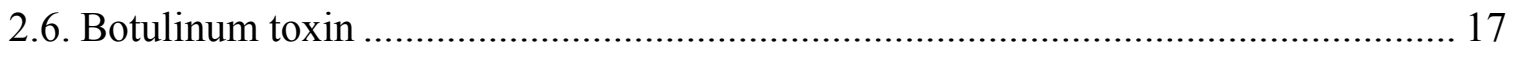

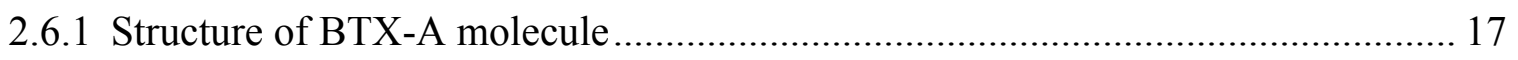

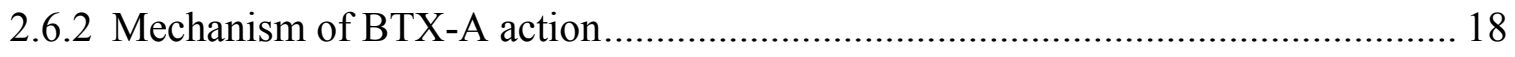

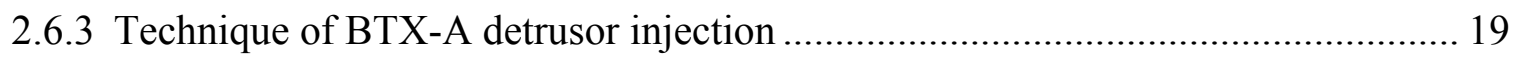

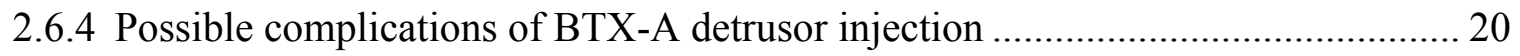

2.6.5 Results of detrusor BTX-A injection in both adults and pediatrics ..................... 21

2.7 Alternative methods of BTX-A delivery: .......................................................... 22

2.7.1 Intravesical BTX-A instillation using liposomes ........................................... 22

2.7.2 Intravesical BTX-A delivery using Electromotive Drug Administration 23

2.7.4 Intravesical BTX-A instillation using Protamine Sulphate: ............................... 24

2.7.5 Intravesical BTX-A instillation using Dimethyl Sulfoxide (DMSO) ................... 26

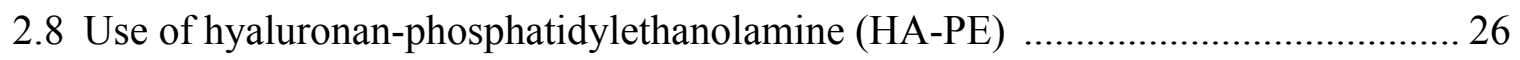

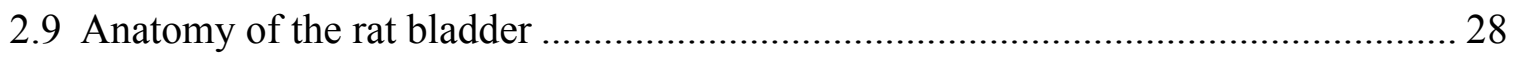

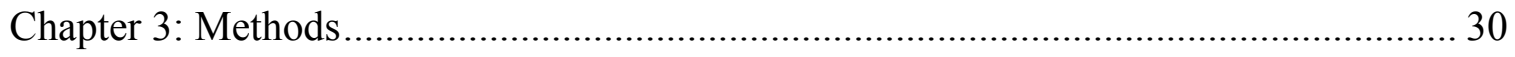

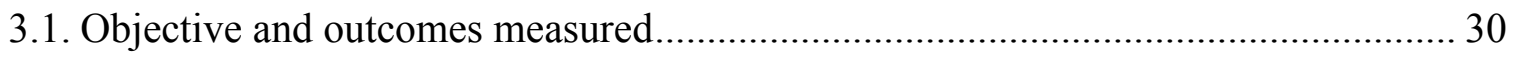

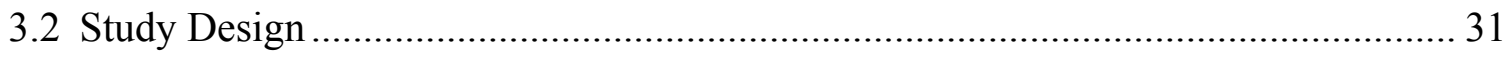

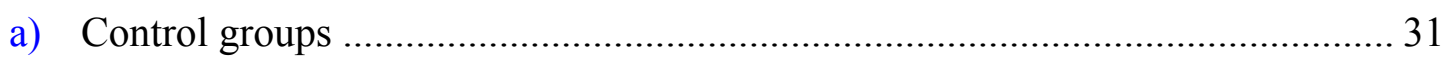

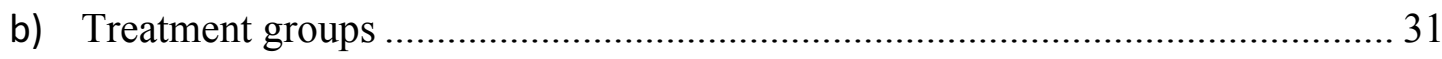

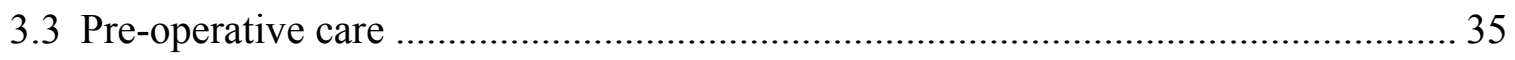

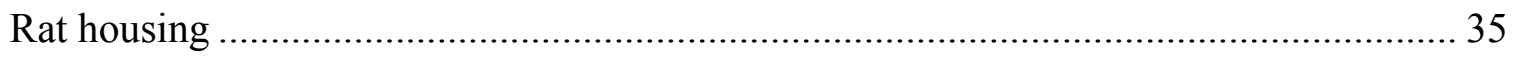

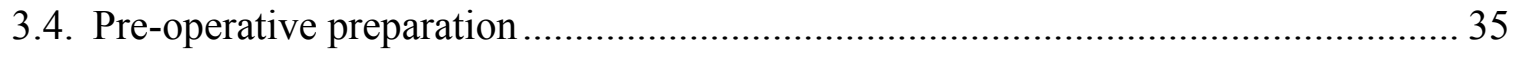

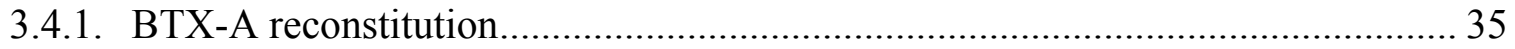




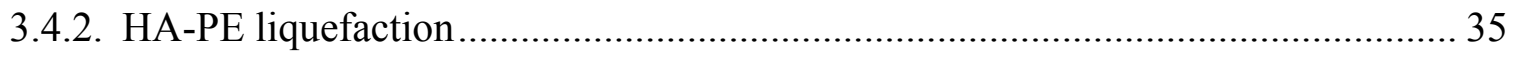

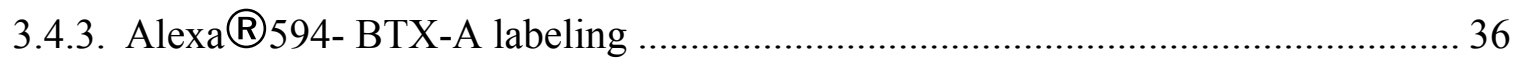

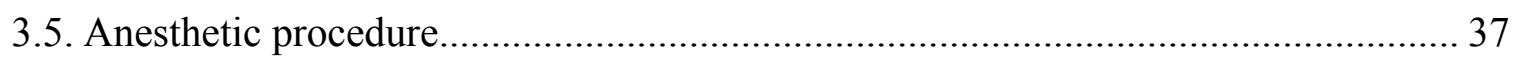

3.6. Surgery procedure and postoperative care ……………………………………..... 39

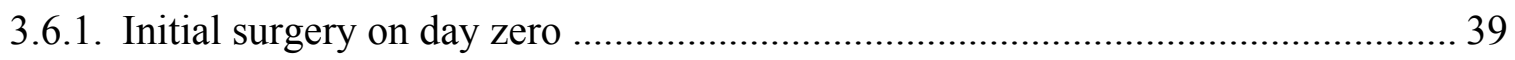

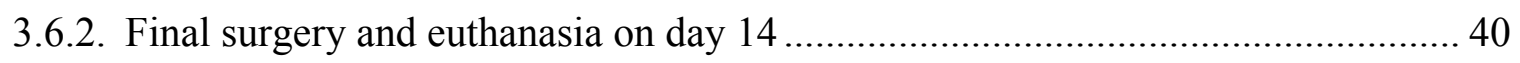

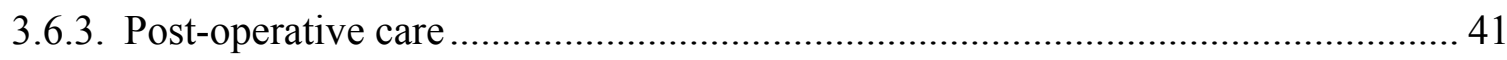

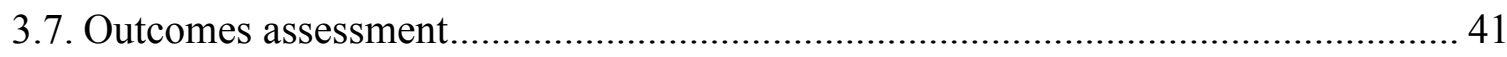

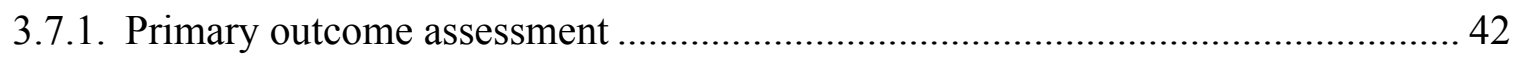

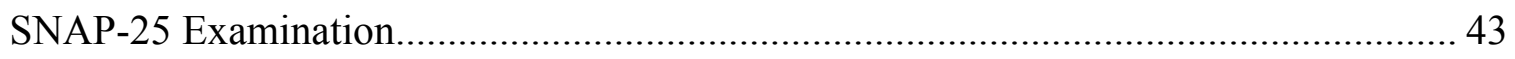

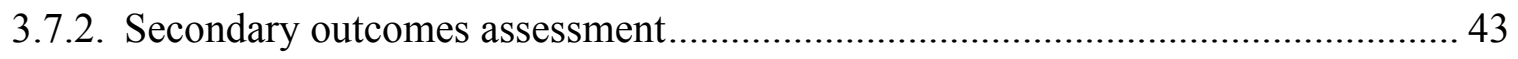

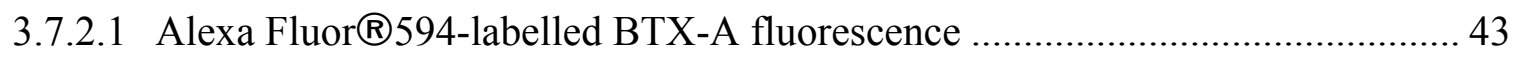

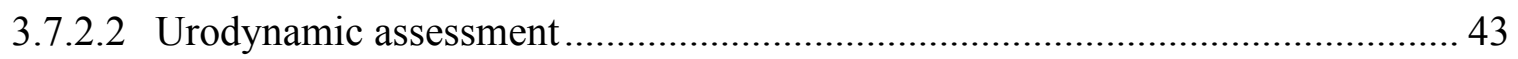

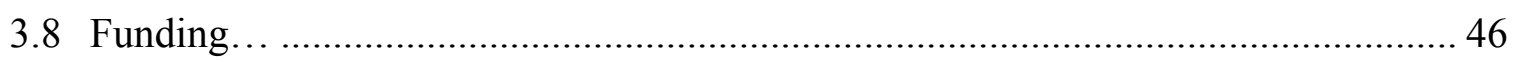

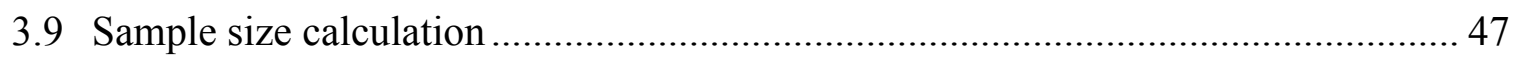

3.10 Data and statistical analysis ............................................................................ 47

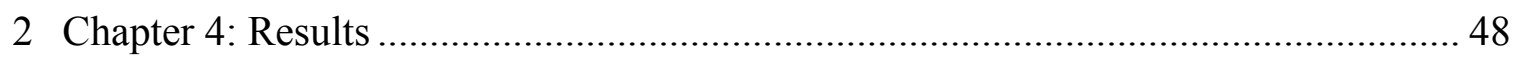

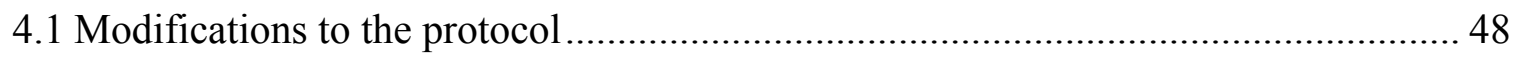

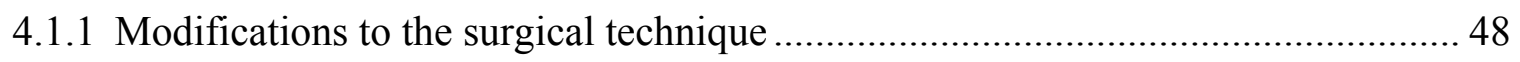

4.1.2 Modifications to the urodynamic assessment …….............................................. 49

4.1.3 Modifications to the injection technique............................................................... 49

4.1.4 Modifications to the BTX-A-HA-PE mixture ……………………………........ 50

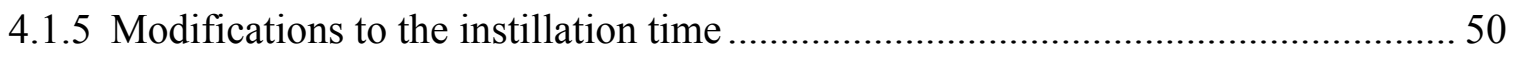

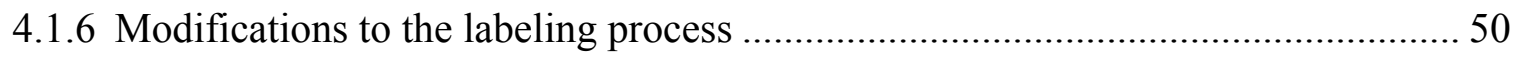

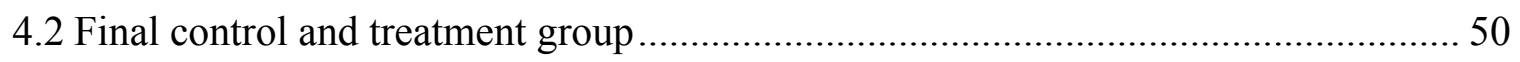




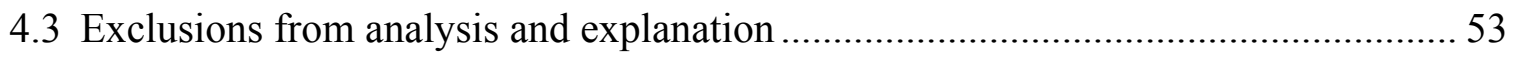

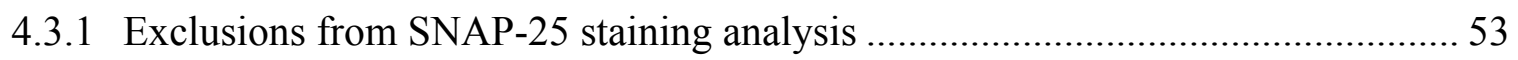

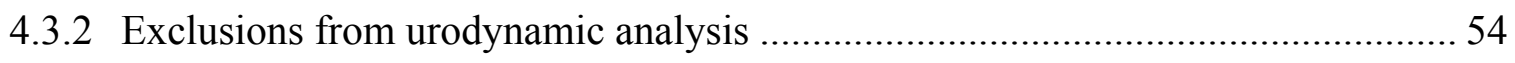

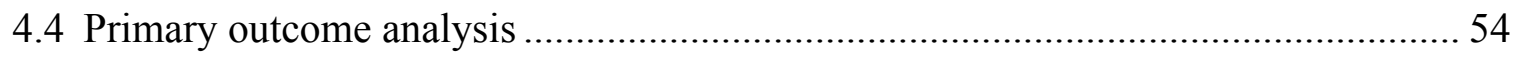

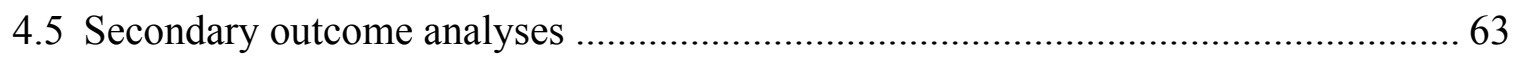

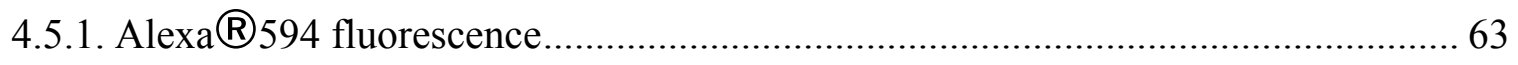

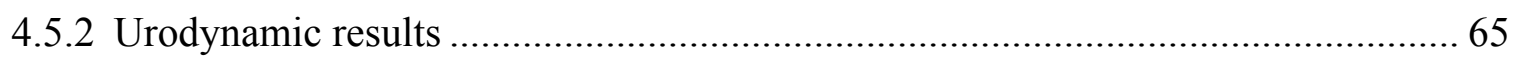

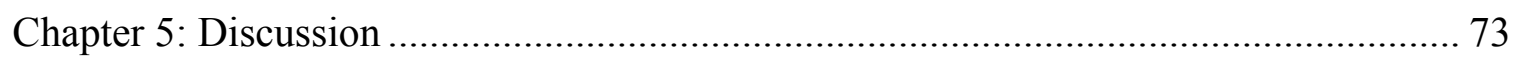

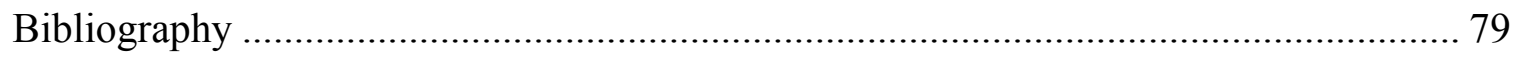

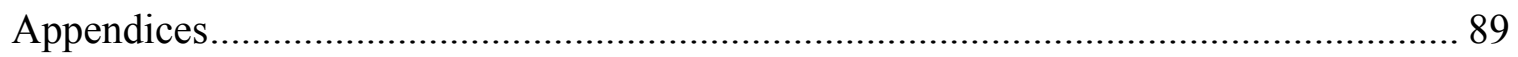

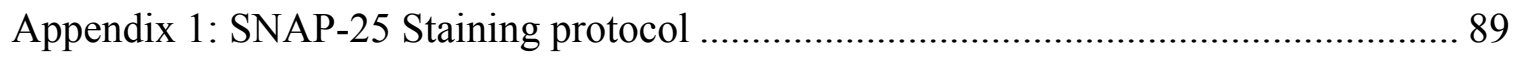

Appendix 2: BTX-A- Alexa Fluor ${ }^{\circledR} 594$ labeling Protocol ......................................... 91

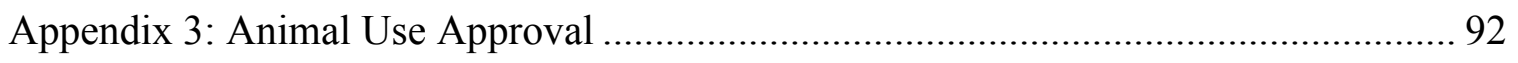

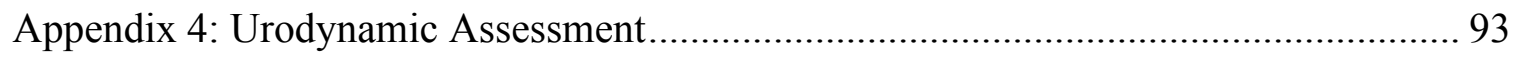

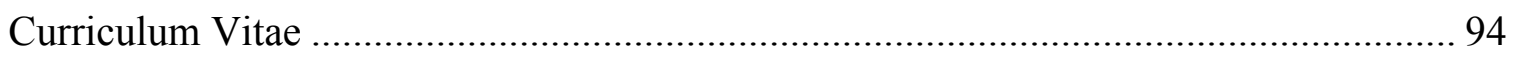




\section{List of Tables}

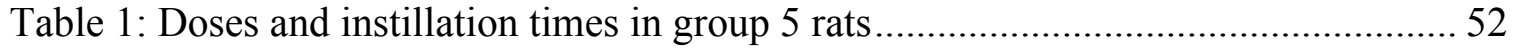

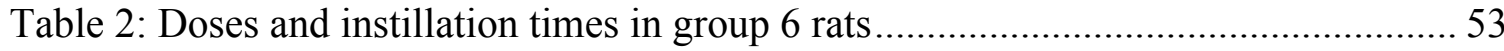

Table 3: Mean SNAP-25 staining percentages \pm SD of in all groups............................. 56

Table 4: SNAP-25 staining percentage comparisons using one (ANOVA) .................... 62

Table 5: Mean maximum pressure at baseline and after AA instillation, \pm SD............... 65

Table 6: Mean inter-contraction intervals (ICI) at baseline and at 14 days..................... 69 


\section{List of Figures}

Figure 1: Sagittal section of the bladder and adjacent viscera in the female $\ldots \ldots \ldots \ldots \ldots \ldots \ldots \ldots . \ldots$

Figure 2: sagittal section of the bladder and adjacent viscera in the male..................... 8

Figure 3: Anatomy of the bladder and its outlet, as defined by Gosling and Dixon...............

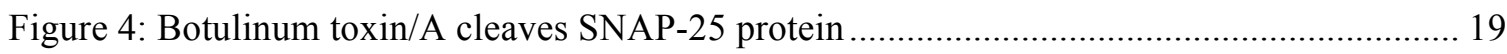

Figure 5: Intra-detrusor injection of Botulinum Toxin A using an injection needle .................... 20

Figure 6: Photomicrographs of sections of the urinary bladder of adult female rats.................... 29

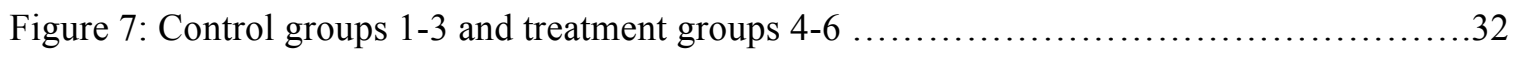

Figure 8: Botulinum Toxin/A- HA-PE- saline instillation, control groups $\ldots \ldots \ldots \ldots \ldots \ldots \ldots \ldots . \ldots \ldots$

Figure 9: Botulinum Toxin A- HA-PE- saline instillation, treatment groups.................... 34

Figure 10: HA-PE its original form as a paste and after liquefaction......................... 36

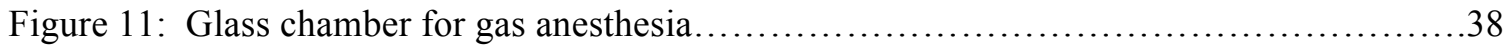

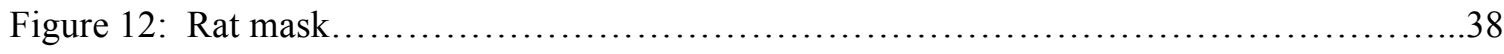

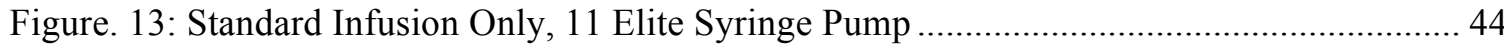

Figure 14: Digi-Med, Micro-Pressure Analyzer................................... 45

Figure 15: Urodynamic study showing the different parameters measured................................46

Figure 16: SNAP-25 staining in treatment and control groups...........................59

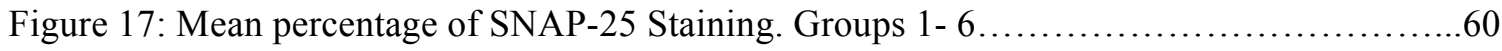

Figure 18: Mean percentage of SNAP-25 Staining. Groups 3,5 and 6..............................61

Figure 19 : Alexa Fluor ${ }^{\circledR 594-l a b e l e d ~ B T X-A ~ b y ~ t h e ~ c o n f o c a l ~ m i c r o s c o p e . . . . . . . . . . . . . . . . . . . .64 ~}$

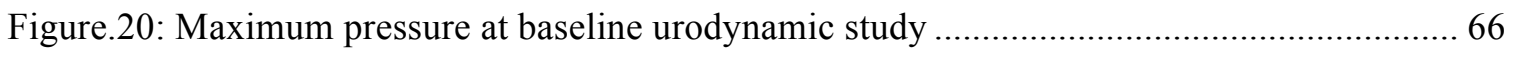

Figure. 21: Maximum pressure after 1\% acetic acid instillation on follow up urodynamic study. 67

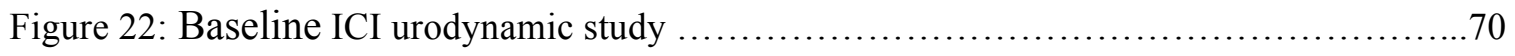

Figure 23: ICI after AA instillation on follow up urodynamic study ........................71 


\section{List of Abbreviations}

ECM: Extra Cellular Matrix

DO: Detrusor Overactivity

QoL: Quality of Life

UI: $\quad$ Urinary Incontinence

SCI: $\quad$ Spinal Cord Injury

MS: $\quad$ Multiple Sclerosis

OAB: Over Active Bladder

NDO: Neurogenic Detrusor Overactivity

CIC: Clean Intermittent Catheterization

BTX-A: Botulinum Toxin - A

HA: Hyaluronic acid

SNAP-25: Synaptosome Associated Protein -25kDa

HA-PE: Hyaluronan- Phosphatidyl-Ethanolamine

GAG: Glycosaminoglycans

M: $\quad$ Muscarinic receptor

a. AR: Alpha Adrenergic Receptor

B. AR: Beta Adrenergic Receptor

TRPV: Transient Receptor potential vanilloid receptor

SPN : $\quad$ Sacral Parasympathetic Nucleus

NANC : Non Adrenergic Non Cholinergic

ATP: Adenosine Tri-Phosphate 
IMG: Inferior Mesenteric Ganglion

PAG: Peri Aquiductal Greymatter

ITP: Inositol Tri- Phospate

DSD: Detrusor Sphincter Dyssynergia

SNARE: Soluble N-ethylmalidemide Attachment Receptor

LESW: Low Energy Shock Wave

SV: $\quad$ Synaptic vesicle

P2X: Purinergic Receptor

CGRP: Calcitonin Gene Related Peptide

EMDA: Electro Motive Drug Administration

MRI: Magnetic Resonance Imaging

GPA: Gadolinium Penta Acetic Acid

COX-2: Cyclo- Oxygenase 2

DMSO: Dimethyl Sulphoxide

PBS: $\quad$ Phosphate Buffered Saline

LRCP: London Regional Cancer Center

DAB: 3, 3'-diaminobenzidine

DAPI: 4', 6-Diamidino-2-Phenylindole

MP: $\quad$ Maximum Pressure

ICI : $\quad$ Inter Contraction Intervals

FITC : Fluorescéine isothiocyanate

UDS: Urodynamic study 


\section{List of Appendices}

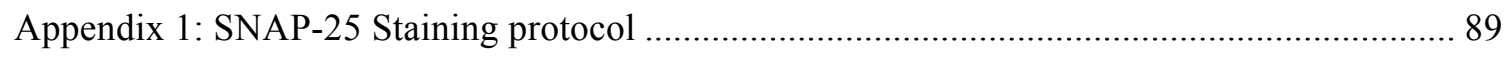

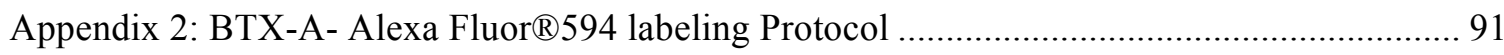

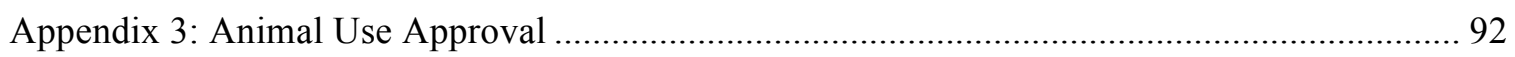

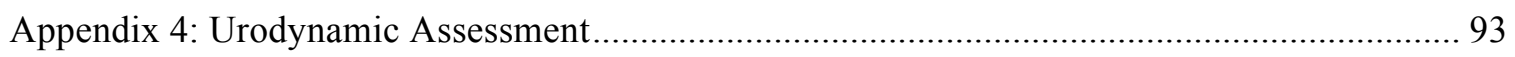




\section{Chapter1 : Introduction}

\subsection{Bladder function and dysfunction}

The urinary bladder is a unique organ. As a hollow structure, it is remarkably integrated in its neuromuscular, mechanical, and physical properties, all of which are crucial for its normal function. The urinary bladder is evolutionarily programmed to fill with fluid over hours at low pressures, and then contract on demand to evacuate its contents. It is also the only autonomic organ under voluntary control.

Although the ability to accommodate a volume of fluid (i.e., filling) is thought to largely represent a passive phenomenon, this is not entirely the case. The recruitment of active neuro-mechanical properties is crucial to the maintenance of low intra-vesical pressures while the bladder fills; and to this end, the characteristics of the surrounding cellular elements and extracellular matrix (ECM) can either facilitate or compromise this function. Bladder contraction (i.e., emptying), on the other hand, is largely an active neuromuscular process, although it is also influenced by the physicochemical nature of the ECM, and obviously depends on the overall mass and optimal functional status of bladder smooth muscle cells (bSMCs).

According to the standardization of terminology for lower urinary tract function published by the International Continence Society (ICS), detrusor overactivity (DO) is a urodynamic phenomenon characterized by involuntary detrusor contractions during the filling phase that may be either spontaneous or provoked (1). DO can occur in patients with neurological disorders and also in those with no underlying neurological anomaly. When there is a relevant underlying neurological condition, this is qualified as neurogenic detrusor overactivity (NDO). In patients with neurological disorders, the prevalence of bladder dysfunction associated with NDO is approximately $12-19 \%$ in both men and women (2-4). 
Overactive bladder $(\mathrm{OAB})$ is a symptom complex described as urgency, with or without urge incontinence, usually associated with frequency and nocturia. Although $\mathrm{OAB}$ is not specific for any condition or urodynamic finding, patients with $\mathrm{OAB}$ are often found to have DO.

Detrusor over-activity impairs quality of life $(\mathrm{QoL})$ secondary to urinary incontinence and poses a threat for the upper urinary tract when associated with high bladder filling pressures. Urinary incontinence (UI) affects approximately one third of patients with an overactive bladder $(3,5)$.

Oral anti-muscarinic agents have been widely used as first-line treatment for patients with DO. Failure of adequate anti-muscarinic therapy, may require surgical intervention designed to reduce bladder storage pressures and inhibit DO. Currently these options include intra-detrusor botulinum toxin injections and bladder augmentation $(6,7)$. Bladder augmentation, which involves using a patch of bowel to increase bladder capacity and thereby lower filling pressures, is an effective surgical option. The ileum is the preferred bowel segment used, but colon and stomach have been utilized as well. Unfortunately, exposing urine to the bowel patch's absorptive surface creates a new set of potential complications and morbidities, like electrolyte and acid base balance disturbances, urinary tract infections, stone formation, the risk of bladder perforation, and malignancy.

\subsection{Botulinum toxin}

Botulinum toxin (BTX), first isolated by van Ermengem in 1897, is a potent neurotoxin produced by the gram-positive anaerobic bacterium Clostridium botulinum (8). There are seven distinct serotypes of BTX (A-G), of which types A and B have been used clinically. BTX-A was first licensed under the brand name Botox ${ }^{\circledR}$ and has been the most commonly used serotype in clinical practice. From a structural viewpoint, the toxin is a $150-\mathrm{kD}$ amino acid di-chain molecule consisting of one light $(50 \mathrm{kD})$ and one heavy chain $(100 \mathrm{kD})$, which are linked by a disulfide bond. 
The role of BTX-A at the neuromuscular junction has been well described and consists of inhibiting acetylcholine neurotransmitter release, resulting in striated muscle relaxation (9). However, increasing evidence suggests that it has a much greater range of neurological effects, as BTX-A has been discovered to inhibit the release of a number of neurotransmitters (including acetylcholine, adenosine triphosphate, and neuropeptides like substance $\mathrm{P}$ ) and to down-regulate the expression of purinergic and capsaicin receptors on afferent neurons within the bladder (10).

In 1989, botulinum toxin A (BTX-A) was approved by the United States Food and Drug Administration (FDA) for use in patients with strabismus and blepharospasm. Since then, its use has been extended to cervical dystonia, cosmesis, hypersecretory disorders and overactive muscle disorders.

Following the publication of studies in adults, Schulte-Baukloh et al, demonstrated the safety and efficacy of intra-vesical BTX-A injections in pediatric patients with neurogenic bladders (NB) (11). Since then, several observational studies have confirmed the significant and impressive response to BTX-A injections in the NB population (1214). However, the current status of this mode of therapy is primarily limited to end-stage bladders as an option to delay bladder augmentation. This therapeutic option requires direct bladder muscle injection under cystoscopic guidance and therapy has to be repeated every 6-9 months. To obviate the need for repeated injections, several authors have studied alternative means of BTX delivery to the detrusor muscle using several carriers (15-19). In addition, alternative modes of BTX-A delivery are preferable, as injections may require anesthesia and are associated with increased costs related to the procedure, and possible complications. Unfortunately, these alternative techniques have not yet yielded a reliable and acceptable means of BTX delivery without injection to the bladder muscle. 


\subsection{Hyaluronic acid-based topical delivery of proteins}

Hyaluronic acid (HA) is a hydrophilic polysaccharide that is ubiquitous in tissues. In its native form (e.g.> $500 \mathrm{kDa}$ ), it is highly viscoelastic $(20,21)$. Continuous HA networks or channels link the layers of the epidermis $(22,23)$.

Small amounts of unmodified HA can cross epidermal layers, likely via its ability to insert into HA coats $(24,25)$. However, most $(>98 \%)$ of the unmodified HA binds only to the skin surface, where it can act as a reservoir for slow-release drugs. The use of HA for drug release is restricted, however, to low molecular weight drugs only (e.g., Solaraze: HA/diclofenac gel).

A non-particulate formulation has been developed that increases the penetration of HA through the epidermis, this was achieved by linking HA to phospholipid chains (PE) and selecting those formulations that promote HA coat formation around keratinocytes. This non-particulate formulation forms HA coats around epidermal cells, and takes advantage of both the high viscosity of HA polymers, which permits the encapsulation of large proteins, and its efficient delivery to the dermis. The high viscosity of these formulations enmeshes large proteins, like BTX-A, which then can be carried with HA-PE into the epidermal layer. Proteins like BTX-A likely accumulate in the lower dermis and muscle layers by slow release or diffusion. Local inflammatory changes and/or systemic toxicity has not yet been observed (26).

\subsection{Objectives of the current study}

The purpose of the current experimental study was to evaluate a novel alternative modality of BTX-A delivery to the detrusor muscle using HA-PE carrier by simple bladder instillation rather than cystoscopic detrusor injection of BTX-A. The secondary objective was to assess our surgical technique and standardize the urodynamic study protocol. 


\subsection{Hypothesis}

We proposed that BTX-A-HA-PE-saline intravesical instillation allows translocation of BTX-A across the urothelium into the bladder muscle. The evidence of this delivery will be shown by the following outcomes assessment:

1. Instilled BTX-A would be observed to act at pre-synaptic terminals and cleave cytosolic SNAP-25 protein, indicated by a decreased level of SNAP-25 staining in detrusor muscle. This decrease in SNAP-25 staining will be comparable to direct BTX-A muscle injection.

2. Bladder instillation of BTX-A labeled with a fluorescent agent (Alexa Fluor®594) would be visualized across the epithelium and in detrusor muscle to demonstrate physical translocation across the urothelium.

3. Further, bladder instillation of BTX-A HA-PE will show a physiological response evidenced by decrease bladder muscle contraction on urodynamic studies in response to a bladder irritant instillation (acetic acid) which will be comparable to the physiological response elicited by direct BTX-A detrusor muscle injection.

\subsection{Outcomes}

\subsubsection{Primary outcome}

The primary outcome of this study was to compare BTX-A-HA-PE intravesical instillation to simple BTX-A-Saline intravesical instillation and to direct BTX-A bladder muscle injection using the mean SNAP-25 staining percentage in the corresponding tissue slides described in section 3.7 


\subsubsection{Secondary outcomes}

1) Assess and modify our surgical technique and standardize the urodynamic study protocol.

2) Detection of Alexa Fluor ${ }^{8} 594$ labeled-BTX-A fluorescence compared to controls to demonstrate physical evidence of BTX-A delivery across the urothelium.

3) Observing the effect of BTX-A-HA-PE intravesical instillation on urodynamic parameters (maximum detrusor pressure and inter-contraction intervals) in treatment groups compared to direct bladder muscle injection of BTX-A and other control groups.

4) Observing the effect of HA-PE dose and duration of intravesical instillation on the efficiency of BTX-A delivery across the urothelium.

5) Detection of toxicity and/or inflammatory changes caused by the HA-PE carrier on the bladder urothelium and musculature. 


\section{Chapter 2: Review of literature \\ 2.1 Anatomy of the urinary bladder}

The bladder is a hollow muscular organ that serves as a reservoir for urine collection and emptying. The bladder is divided into two parts; a body that lies above the ureteral orifices and a base that consists of the trigone and the bladder neck (27). The bladder outlet is composed of bladder base, urethra and external urethral sphincter.

The bladder base has a laminar architecture with a superficial longitudinal layer that lies beneath the trigone. A muscle layer deep to the superficial layer is continuous with the detrusor muscle (28-30). The smaller muscle bundles of the deep muscle layer within the bladder base predominantly exhibit a circular orientation.

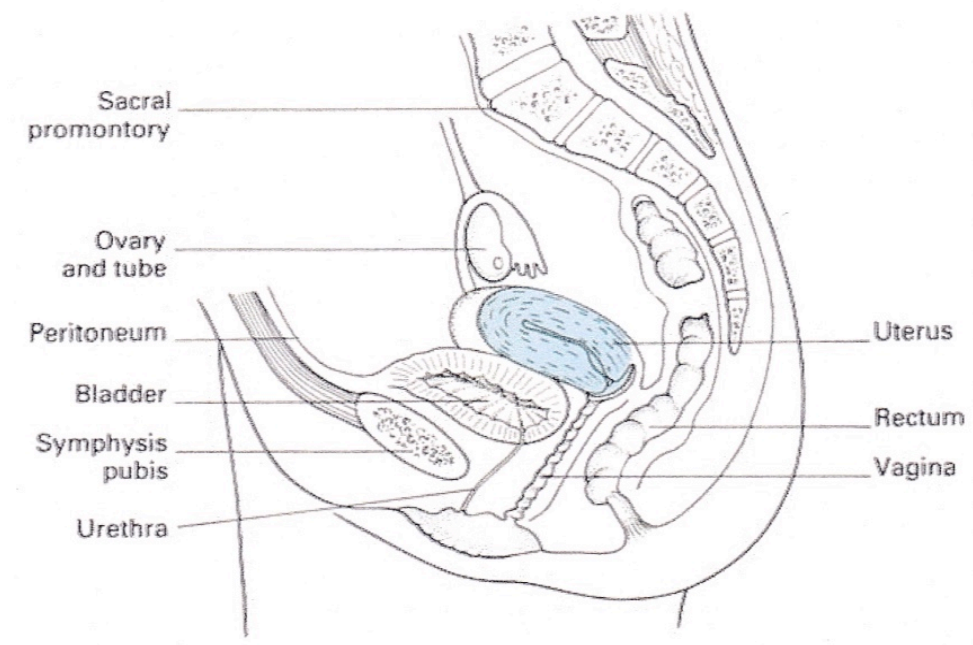

Figure 1: Sagittal section of the bladder and adjacent viscera in the female 


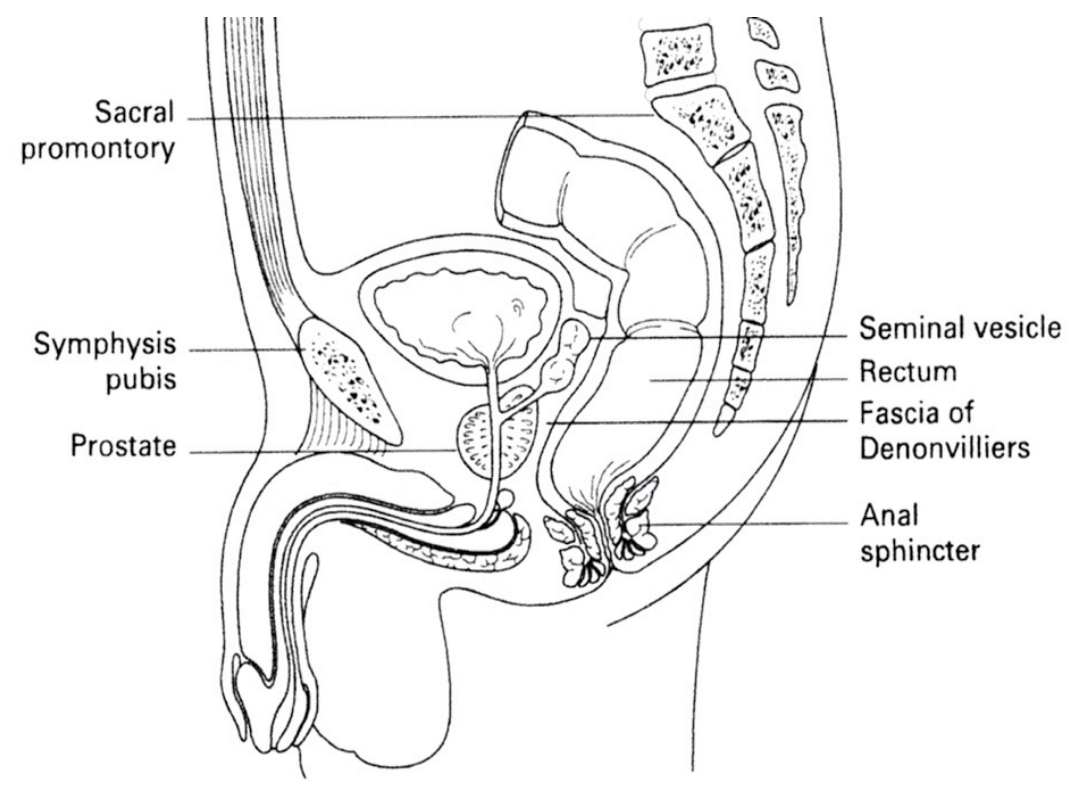

Figure 2: sagittal section of the bladder and adjacent viscera in the male

The triangle of smooth urothelium between the two ureteral orifices and the internal urethral meatus is referred to as the trigone of the bladder. The fine longitudinal smooth muscle fibers from the vesical side of the ureters pass to either side of their respective orifices to join the lateral and posterior ureteral wall fibers, where they then fan out over the base of the bladder. Fibers from each ureter meet to form a triangular sheet of muscle that extends from the two ureteral orifices to the internal urethral meatus. The edges of this muscular sheet are thickened between the ureteral orifices (the inter-ureteric crest or Mercier's bar) and between the ureters and the internal urethral meatus (Bell's muscle). 


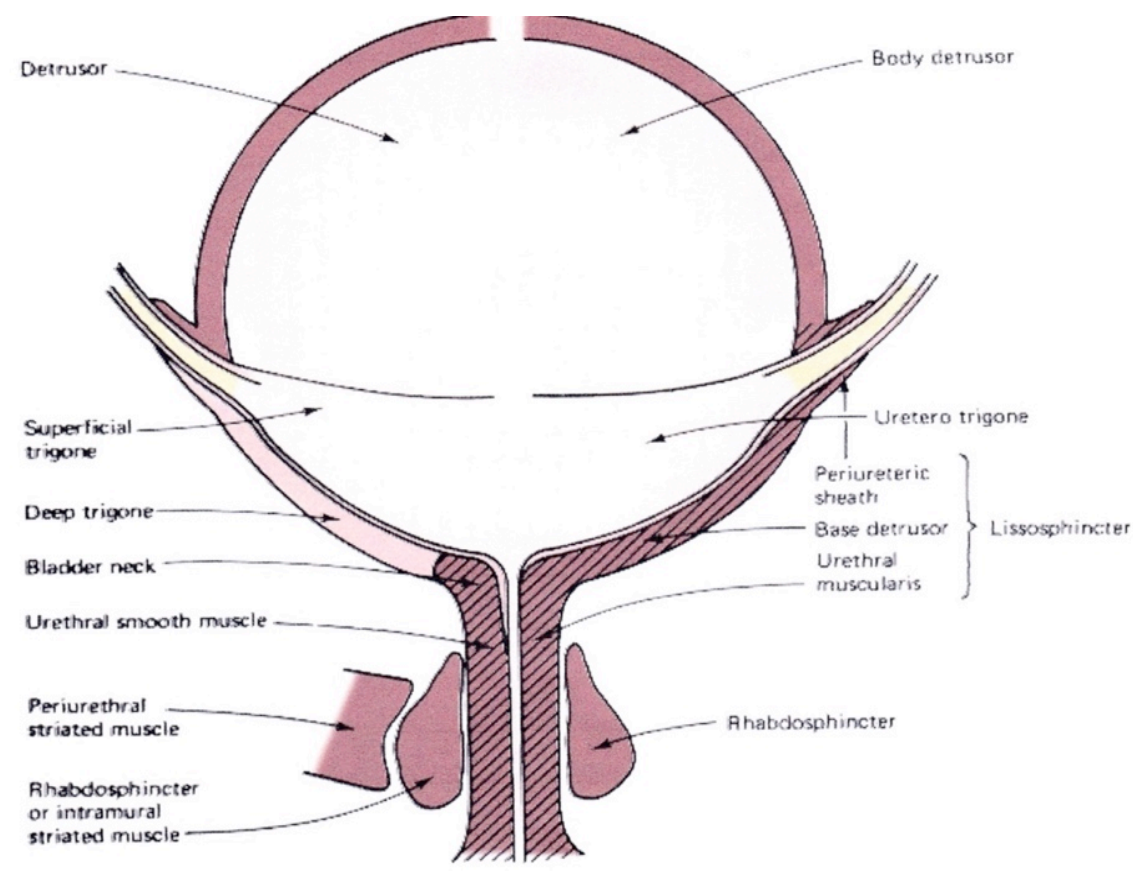

Figure 3: Anatomy of the bladder and its outlet, as defined by Gosling and Dixon (left) versus Elbadawi et al (right). (From Torrens M, Morrison JFB: The Physiology of the Urinary Bladder. Berlin, Springer-Verlag, 1987:1).

\section{Blood supply:}

Blood to the bladder is supplied by the superior and inferior vesical branches of the internal iliac artery. The vesical veins form a plexus that drains into the internal iliac vein.

\section{Lymphatic drainage:}

Lymph vessels drain alongside the vesical blood vessels to the iliac and then the paraaortic nodes.

\section{Nerve supply:}

Parasympathetic fibres from S2 to S4 accompany the vesical arteries to the bladder. They convey motor fibres to the muscles of the bladder wall and inhibitory fibres to its internal sphincter. 
Sympathetic efferent fibres are inhibitory to the bladder muscles and innervate motor function at its sphincter, though they may be mainly vasomotor in function, so that normal filling and emptying of the bladder are probably controlled exclusively by its parasympathetic innervation. Sensory fibres from the bladder, which are stimulated by distension, are conveyed in both the sympathetic and parasympathetic nerves, the latter pathway being the more important. The external sphincter is made up of striated muscle. It is also concerned with the control of micturition and is supplied by the pudendal nerve $(\mathrm{S} 2,3,4)$.

\subsection{Architecture of the bladder wall}

The bladder wall consists of three distinct tissue layers. These layers, starting from the inner luminal surface and progressing outwards, are: the mucosa; the muscularis propria; and the adventitia/serosa. The mucosa in turn, consists of three layers: the urothelium, the basement membrane, and the lamina propria. The urothelium is approximately six cells thick, creating a transitional epithelium that consists of so-called 'umbrella cells' that form the luminal surface, with underlying intermediate cells, and finally, basal cells that rest upon a thin basement membrane $(31,32)$.

It is the urothelium and specifically the umbrella cells that render the bladder impermeable, thereby preventing urine and waste solute from penetrating into the submucosa. The barrier function of umbrella cells stems from an arrangement of tight junctional proteins called uroplakins, and is further augmented by an overlying-luminalmucin layer composed of glycosaminoglycans (GAGs). Suggested additional role of this GAG layer is to prevent particulates in the urine from adhering to the bladder lumen (33).

One of the most remarkably distinct characteristics of the bladder is its elasticity. This elasticity is so great that, whereas the transitional epithelium appears smooth when the bladder is full, it contracts into numerous folds when the bladder empties. The bladder wall's elasticity allows it to store $400-600 \mathrm{ml}$ of urine in the average-weight human adult under low pressure. 
This elasticity is the result of both the lamina propria — the deepest layer of mucosa and the detrusor muscle that underlies it. The lamina propria is a relatively thick layer of fibroelastic connective tissue and smooth muscle fibers collected into a poorly-defined muscularis mucosa that together allow for considerable distension. This layer is also traversed by numerous blood vessels.

Beneath this layer lies the smooth muscle of the bladder wall, called the detrusor muscle. There, large muscle fibers form branching, interlacing bundles loosely arranged into inner longitudinal, middle circular, and outer longitudinal layers. The outer longitudinal fibers are thickest posteriorly at the bladder base. In the midline, they insert into the apex of the trigone and intermix with the smooth muscle of the prostate to provide strong trigonal support. Laterally, the fibers from this posterior sheet pass anteriorly and fuse to form a loop around the bladder neck.

In the bladder neck of males, radially-oriented inner longitudinal fibers pass through the internal meatus to become continuous with the inner longitudinal layer of smooth muscle in the urethra. With respect to the inner longitudinal fibers, this is similar in females. However, the middle circular layer in females does not appear to be as robust as in males. Where female bladder necks differ most strikingly from that of males in its scanty adrenergic innervation. In addition, its sphincter function is limited, such that, in $50 \%$ of continent women, urine still enters the proximal urethra when they cough (34).

\subsection{The blood-urine barrier}

The urothelium is the most impermeable barrier in the body (35). It also is responsible for a multitude of physiological activities, not only effective at blocking the passage of urine and its contents, but equally effective at blocking the passage of bladder-instilled drugs $(36,37)$. It sits at the interface between the urine and underlying connective tissue, where it forms a barrier preventing the unregulated exchange of solutes, ions and toxic metabolites. The permeability of substances like water, ammonia and urea, which normally cross membranes relatively rapidly, is extremely low across the urothelium $(35,36)$. 
Indeed, the GAG layer might play a greater role preventing bacterial adherence, as described by Hanno et al (1981) (38). The GAG layer might also be important at preventing the formation and attachment of particulates to the urothelium, and thereby, subsequent stone formation (39). This being said, there are a number of problems with the theory that the GAG layer is a major urothelial-plasma barrier, which are beyond the scope of this thesis.

\subsection{Physiological aspects of bladder function}

The lower urinary tract differs from other visceral structures in several ways. Its dependence on CNS control distinguishes it from the structures that maintain a level of function even after all extrinsic neural input has been eliminated. It is also unusual in its pattern of activity and in the organization of its neural control mechanisms.

\subsubsection{Muscarinic receptors of the urinary bladder}

There are five subtypes of muscarinic receptor, which have been labelled M1 through M5. Encoded by five distinct genes and present in both animal and human bladders, the mRNAs for all muscarinic receptor subtypes have been demonstrated, albeit with a predominance of mRNAs encoding for M2 and M3 receptors.

Detrusor muscle contains muscarinic receptors mainly of the M2 and M3 subtypes, with the M3 receptors in human detrusor muscle the most important for muscle contraction (40). It has been suggested that M2 receptors may oppose sympathetically-mediated smooth muscle relaxation mediated by $\beta$ adrenergic receptors (41).

M2 receptor stimulation might also activate nonspecific cation channels and inhibit KATP via the activation of protein kinase $\mathrm{C}$. Thus, in a denervated rat bladder, a combination of M2 and M3 receptors mediates contractile responses. Both types of receptor seem to act in a facilitatory manner to mediate contraction (42). Muscarinic receptors have also been located at pre-synaptic nerve terminals and to participate in the regulation of transmitter release. 
In addition, they have been demonstrated in the urothelium and sub-urothelium and may be directly involved in afferent signaling $(43,44)$.

\subsubsection{Adrenergic receptors of the urinary bladder}

$\alpha$ - adrenergic receptors $(\alpha \mathrm{AR})$ :

Most investigators agree that there is low-level expression of $\alpha \mathrm{ARs}$ in the human detrusor muscle (45). Two types of $\alpha$ receptor are known to exist $-\alpha 1 \mathrm{~A}$ and $\alpha 1 \mathrm{D}-$ and some investigators have noticed increased $\alpha \mathrm{AR}$ expression in cases of outflow obstruction. However, this remains a subject of debate (46).

\section{ß-adrenergic receptors:}

All three types of $\beta$ receptor $(\beta 1, \beta 2, \beta 3)$ can be found in the detrusor muscle of most species, including humans (45). Clinical and functional evidence indicate the predominance of $\beta 3$ receptors in both normal and neurogenic bladders (45). The human detrusor also contains $\beta 2-\mathrm{AR}$ and both receptors, $\beta 2$ and $\beta 3$, are most likely involved in the process of bladder relaxation $(45,47)$. B3-AR has a relaxing effect on the detrusor, which may be the basis for its therapeutic effects in detrusor overactivity (DO).

\subsubsection{Vanilloid receptors}

The transient receptor potential (TRP) channel superfamily has been demonstrated to be involved in nociception and mechanosensory transduction in various organ systems. Studies of the lower urinary tract have indicated that several TRP channels — including TRPV1, TRPV2, TRPV4, TRPM8 and TRPA1 - are expressed in the bladder and may act as sensors of stretch and/or chemical irritation. The rationale behind the intravesical instillation of vanilloids (compounds that possess a vanillyl group) is based on the involvement of C-fibres in the pathophysiology of conditions like bladder hypersensitivity and neurogenic detrusor overactivity (NDO). 
In the healthy human bladder, C-fibres carry responses to noxious stimuli, but they are not implicated in the normal voiding reflex. C-fibre bladder afferents proliferate within the sub-urothelium and become sensitive to bladder distension. These changes lead to the emergence of a new C-fibre-mediated voiding reflex, which is strongly involved in spinal neurogenic detrusor overactivity. Improvement in this condition is accomplished by defunctionalizing C-fibre bladder afferents with intravesical vanilloids (48).

\subsubsection{Neural circuits controlling bladder storage and elimination}

Both bladder filling and elimination are controlled by neural circuits in the brain, spinal cord and neural ganglia. Supra-pontine influences are believed to act as on-off switches to shift the lower urinary tract between the two modes of operation: filling and elimination. Both filling and elimination involve a complex pattern of afferent and efferent signaling in parasympathetic (pelvic nerves), sympathetic (hypogastric nerves) and somatic (pudendal nerve) pathways. The next three sections briefly examine each of their roles.

\section{Parasympathetic pathways:}

Preganglionic parasympathetic neurons transmit impulses to the sacral parasympathetic nucleus (SPN) in the spinal cord at the level of S2-S4. Ganglionic neurotransmission is predominantly mediated by acetylcholine acting on nicotinic receptors, although the transmission can also be modulated by adrenergic, muscarinic, purinergic or peptidergic presynaptic receptors. A non-adrenergic, non-cholinergic (NANC) contractile component is identified regularly in the bladders of most animal species. Such a component can also be demonstrated in functionally and morphologically altered human bladder tissue (49).

Adenosine triphosphate (ATP) is the most important mediator of NANC contractions, although the involvement of other mediators cannot be ruled out. The pelvic nerve also conveys parasympathetic fibers to the bladder neck and urethra and exerts an inhibitory effect on the smooth muscle by releasing nitric oxide and other transmitters (40). 


\section{Sympathetic pathways:}

Sympathetic innervation of the bladder and urethra originates from the intermediolateral nuclei in the thoracolumbar region (T10-L2) of the spinal cord. Axons leave the spinal cord via the splanchnic nerves and travel either through the inferior mesenteric ganglia (IMG) and the hypogastric nerve, or pass through the paravertebral chain to the lumbosacral sympathetic chain ganglia and enter the pelvic nerve.

Ganglionic sympathetic transmission is predominantly mediated by acetylcholine acting on nicotinic receptors. In addition, the sympathetic innervation inhibits parasympathetic pathways at both the spinal and ganglionic level. This being said, the importance of sympathetic innervation in relaxation of the human detrusor has not yet been established.

\section{Somatic pathways:}

Somatic innervation of the urethral rhabdosphincter and some perineal muscles (e.g., compressor urethrae and urethrovaginal sphincter) is provided by the pudendal nerve.

These fibers originate from sphincter motor neurons located in the ventral horn of the sacral spinal cord (levels S2-S4) in the region of Onuf's (Onufrowicz's) nucleus (50).

\section{Sensory (afferent) pathways:}

The afferent nerves to the bladder and urethra originate in the dorsal root ganglia at the lumbosacral level of the spinal cord and travel via the pelvic nerve to the periphery. Some afferents originate in dorsal root ganglia at the thoracolumbar level and travel peripherally within the hypogastric nerve $(51,52)$.

The afferent nerves to the striated muscle of the external urethral sphincter travel in the pudendal nerve to the sacral region of the spinal cord. 
The afferents most crucial to the micturition process are myelinated A $\delta$-fibers and unmyelinated $\mathrm{C}$-fibers that travel within the pelvic nerve to the sacral spinal cord, conveying information from receptors in the bladder wall.

The $A \delta$ - fibers respond to passive distension and active contraction, thereby conveying information about bladder filling. The activation threshold for A $\delta$ - fibers is $5-15 \mathrm{~mm}$ $\mathrm{H} 2 \mathrm{O}$. This is the intravesical pressure at which humans report their first conscious sensation of bladder filling. C-fibers have a higher (i.e., less sensitive) mechanical threshold and primarily respond to chemical irritation of the bladder urothelium or to cold. The cell bodies of $\mathrm{A} \delta$-fibers and $\mathrm{C}$-fibers are located in the dorsal root ganglion at the level of the S2-4 and T11-L2 spinal segments. Following chemical irritation, the Cfiber afferents exhibit spontaneous firing when the bladder is empty and increased firing during bladder distension. These $\mathrm{C}$-fibers are usually inactive and are, hence, termed 'silent fibers'. Afferent information about the amount of urine in the bladder is continuously conveyed to the mesencephalic periaqueductal gray matter (PAG), and from there to the pontine micturition center, also called Barrington's nucleus (53).

\subsubsection{Bladder contraction and relaxation}

Contraction of bladder smooth muscle cells is mainly the result of interactions between the contractile proteins actin and myosin, in a manner similar to other muscle cells in the body. In an energy-dependent process, a multi-step enzymatic reaction allows for the cross-bridging and attachment of these two proteins in a cyclical fashion, following the same general scheme proposed for skeletal muscle. ATP is the immediate energy substrate, and is crucial for membrane pump Ca2+ handling, phosphorylation events, and cross-bridge cycling.

\subsubsection{Neurotransmitters and modulators}

In response to acetylcholine released from parasympathetic nerve terminals, muscarinic M3 receptors are thought to induce detrusor muscle contractions by calcium entry through nifedipine-sensitive L-type Ca2+ channels (54-57). 
In addition, increased poly-phosphoinositide hydrolysis results in inositol 1, 4, 5trisphosphate (IP3) production and the release of intracellular calcium stores $(58,59)$.

Calcium also activates a variety of cellular responses when it enters the cytoplasm of a cell by means of transmembrane channels. To be effective as a signal, its concentration must be returned to sub micro-molar levels, driven by ATP pumps. The $\mathrm{Ca} 2+$ pump is a membrane-bound, $\mathrm{Ca} 2+$-activated ATPase, similar to the $\mathrm{Na}+-\mathrm{K}+$ pump that controls ion balance and membrane potential in all animal cells. These pumps belong to a superfamily of ATPases known as the P type, because they depend on the auto phosphorylation using ATP — of conserved aspartic acid residues.

\subsection{Current treatment for neurogenic bladder}

Clean intermittent catheterization (CIC) in combination with anticholinergic medications is considered the standard therapy for children with neurogenic bladder dysfunction associated with neurogenic detrusor overactivity (NDO), poor bladder compliance, and/or detrusor sphincter dyssynergia (DSD) $(58,59)$. Anti-cholinergic medications reduce detrusor over activity and lower bladder storage pressures, while CIC ensures complete bladder emptying and lowers voiding pressures generated secondary to DSD. Failure of anti-cholinergic therapy to lower bladder filling pressure and/or alleviate NDO with risk of upper renal tract deterioration and/or incontinence leads to surgical intervention in the form of Botulinum toxin intra-detrusor injections or augmentation cystoplasty as a final resort.

\subsection{Botulinum toxin}

\subsubsection{Structure of BTX-A molecule}

Botulinum toxins are neurotoxins produced by the facultative gram-positive anaerobic bacteria Clostridium botulinum. They were first isolated by van Ermengem in 1897; but it was Schurch et al, in 2000, who pioneered the use of botulinum toxin A injection into the detrusor muscle as an option to treat bladder overactivity secondary to spinal cord injury (60). Botulinum A toxin (BTX-A) is a two-chain $150 \mathrm{kDa}$ molecule that includes one heavy $(100 \mathrm{kDa})$ and one light $(50 \mathrm{kDa})$ chain linked by a di-sulphide bond. 
The process of binding to a membrane receptor and internalization, a process called endocytosis, is mediated by the heavy chain $(100 \mathrm{kDa})$.

\subsubsection{Mechanism of BTX-A action}

Shortly after internalization, the disulfide bond unravels releasing both the heavy and light chains (61). The light chain then binds with high specificity to the SNARE (soluble N-ethylmaleimide- sensitive factor attachment protein receptor) protein complex, which leads to the cleavage of (25 kDa) SNAP-25, a protein integral to the successful docking and release of acetylcholine from vesicles situated within nerve endings, in a process called exocytosis.

The toxins disrupt different parts of the SNARE receptor, with BTX-A blocking the release of acetylcholine into the synaptic gap of the neuromuscular junction by cleaving the synaptosome-associated protein, SNAP 25 (62-64). This causes selective, temporary flaccid paralysis of the target organ. In smooth muscles, it has been documented to trigger the release of nitric oxide that diffuses out of the endothelial cell and causes smooth muscle relaxation.

The receptor molecule responsible for toxin binding and internalization has been identified as the synaptic vesicle protein 2 (SV2) receptor $(64,65)$. Recent evidence points at another possible mechanism of action for BTX-A, implying that it might affect afferent pathways via some dual mechanism of action (66). The bladder afferent neuronal receptors implicated include vanilloid, purinergic $(\mathrm{P} 2 \mathrm{X})$, and neurokinin receptors for nerve growth factor $(66,67)$. Neurotransmitters acting at these receptors include ATP, substance $\mathrm{P}$, neurokinin A, nitric oxide, and calcitonin-gene-related peptide (CGRP), which modulate the sensory afferent nerves in the detrusor muscle, especially in diseased neurogenic bladders with NDO. The role of the sub-urothelial myofibroblast cells acting as a sensory organ in regulating bladder overactivity and the effect of BTX-A on these cells is also being recognized (68). 

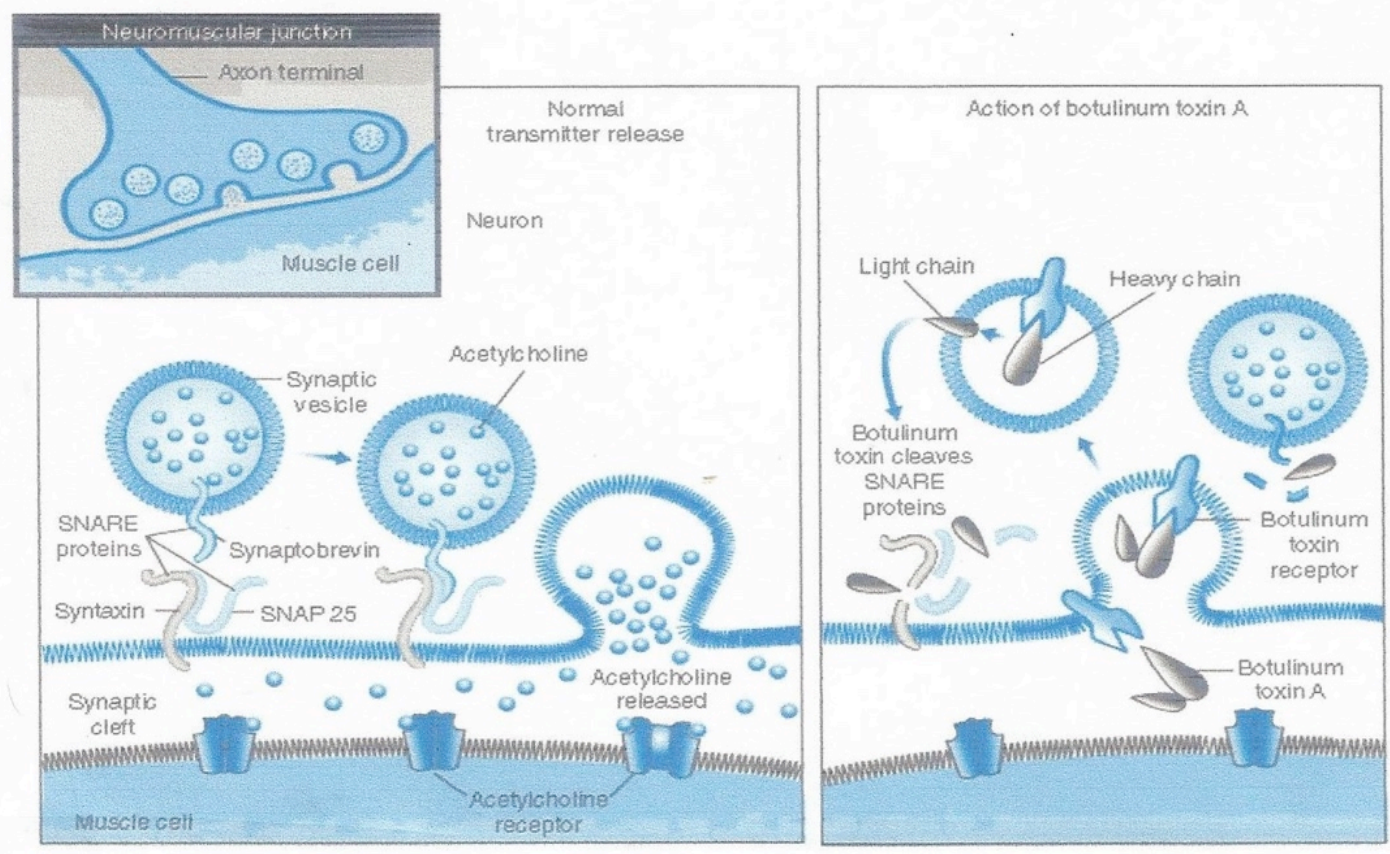

Figure 4: Botulinum toxin/A cleaves SNAP-25 protein, preventing assembly of the fusion complex and thereby blocking the release of acetylcholine (Adopted from Rowland, 35 with permission).

\subsubsection{Technique of BTX-A detrusor injection}

Procedurally, BTX-A injections are performed via cystoscopy and involve its injection directly into bladder muscle at multiple sites (e.g., 20-30), except usually the trigone. Anaesthesia is usually required to complete the procedure, (Fig.5). 


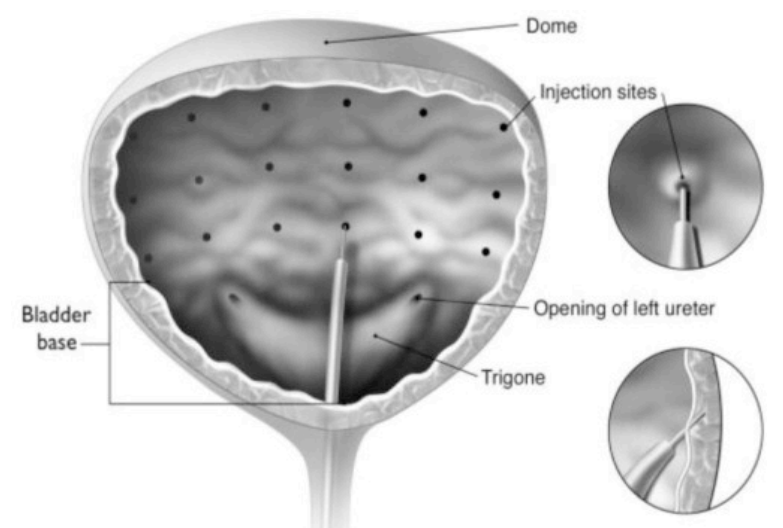

Figure 5: Intra-detrusor injection of Botulinum Toxin A using an injection needle

\subsubsection{Possible complications of BTX-A detrusor injection}

Since its initial use, the efficacy and safety of BTX-A injections to treat detrusor over activity and improve bladder compliance has been well established in both the adult and pediatric scientific literature (69-71). However, BTX-A injection therapy has certain limitations and complications. Apart from the anaesthetic complications; other complications might be a concern including drug leakage outside the bladder and systemic absorption, haematuria, pain at the injection site, and the uneven distribution of botulinum toxin $(72,73)$. As a result of BTX-A injection and SNAP-25 cleavage, chemical denervation results. However, axonal sprouting may then occur, and extrajunctional acetylcholine receptors may develop over time. Consequently, the effects of Botox generally only last six to nine months, such that repeat injections are almost always needed to maintain the drug's therapeutic effects. The use of anaesthesia is usually required for BTX-A injections

Fortunately, since BTX-A cleaves the cytosolic translocation protein SNAP-25, reduced total SNAP-25 due to cleavage can be used as a marker of toxin diffusion and its local action (74), allowing for some assessment of the effectiveness of such alternative BTX-A delivery methods. 
The effect on bladder contractility is also mediated by the suppression of sensory neurotransmission through mechanisms that modulate mediator release from afferent nerve terminals. BTX-A- saline solution instillation has been attempted in animals and humans with limited success on detrusor storage pressures, despite the use of other carriers like liposomes and DMSO, pre-treatment with protamine sulfate to enhance absorption, and the recent employment of low energy shock wave (LESW) therapy to facilitate BTX-A delivery (75-78).

\subsubsection{Results of detrusor BTX-A injection in both adults and pediatrics}

Of the seven distinct but structurally similar serotypes of botulinum toxin, types A and B have been used with clinically beneficial outcomes in various neurological disorders. In children, botulinum toxin A (BTX-A) is used worldwide and is becoming a standard in the treatment of spasticity secondary to cerebral palsy. No systemic side effects have been reported when the appropriate dose is used (79).

In 2009 Game et al, based on the results of a systematic literature review of 6 clinical studies involved 108 children, evaluated the efficacy and safety (or both) of BTX-A in children with NDO/NOAB and subsequent clinical expert discussion of the outcomes, this review provided more insight into these topics on an evidence-based medicine level. They concluded that, in children with NDO/NOAB resistant to anti-muscarinics, BTX-A intradetrusor injection have a clinically significant effect. Treatment with BTX-A seemed to be well tolerated with minimal injection site and systemic side effects (70).

Karsentley G, et al; 2008 based on the results of a systematic literature review of 18 clinical studies involved 698 adults, evaluated the efficacy or safety of BTX-A in adult population. Of all adult patients included, 83\% had NDO with urinary incontinence and/or NOAB (mainly due to SCI 57\% and MS 17\%) and refractory to usually high doses of antimuscarinic agents. Only three studies also enrolled patients with idiopathic DO (IDO) or bladder outlet obstruction (BOO) (71). They concluded that, treatment with BTX-A intradetrusor injections provide a clinically significant benefit to adults with $\mathrm{NDO} / \mathrm{NOAB}$ resistant to antimuscarinics. 
The onset of effect is fast (within 1-2 wk.) and after reinjection (time interval between injections approximately 6-9 months), the effect on the clinical and urodynamic variables persists. Moreover, treatment with BTX-A seems to be very well tolerated with minimal injection site and systemic side-effects (71).

\subsection{Alternative methods of BTX-A delivery}

\subsubsection{Intravesical BTX-A instillation using liposomes}

An animal experiment on Sprague Dawley rats divided into 3 groups was reported comparing intravesical instillation of $20 \mathrm{U}$ BTX-A plus $1 \mathrm{ml}$ liposomes (Lipotoxin) retained in the bladder for one hour to BTX-A toxin and liposomes (LP) instilled alone. Outcomes measured included the extent of SNAP-25 cleavage and the expression of calcitonin-gene related peptide (CGRP) in harvested bladders. Urodynamic functional parameters were measured at baseline and 8 days later after instillation of $0.3 \%$ acetic acid (80).

The authors found that intravesical lipotoxin suppressed acetic acid induced bladder hyper-activity and inflammatory reaction, which was not observed in LP pre-treated groups and was less significant in BTX-A pre-treated groups. Furthermore, SNAP-25 expression was significantly decreased and CGRP was significantly increased in the lipotoxin pre-treated group compared to those in the LP and BTX-A pre-treated groups. Importantly, bladder contraction amplitude did not show a significant change between days 1 and 8 baseline UDS and urinary retention was not seen in the lipotoxin pre-treated group. Therefore, their results support the concept that intravesical lipotoxin instillation may be a simpler, effective method to deliver BTX-A across the urothelium without injection. Caution must be exercised interpreting these results, however, especially since neither the total number of used rats, nor the number of rats in each group were provided. 


\subsubsection{Intravesical BTX-A delivery using Electromotive Drug Administration (EMDA)}

In an experimental study, 15 male healthy New Zealand white rabbits were allocated into 3 groups; BTX-A injection into the bladder wall, intravesical electromotive BTX-A administration (BTX-A/EMDA), and electromotive saline administration $(81,82)$. In BTX-A injection group, a total dose of $10 \mathrm{IU} / \mathrm{kg}$ of BTX-A was injected into 10 sites of bladder detrusor muscle using a 6-Fr rigid cystoscope. In BTX-A/EMDA group, a current generator delivered a total of 2-2.4 mA with a frequency of $2.5 \mathrm{kHz}$ to a fully distended bladder containing $10 \mathrm{IU} / \mathrm{kg}$ BTX-A for 15 minutes. In electromotive saline administration group, electrical current with the same characteristics was delivered to a saline-filled bladder. Three different specimens from the bladder dome, posterior, and anterior bladder walls were obtained and submitted for pathologic evaluation as frozen sections.

For the immunohistochemistry, sections were treated with primary anti-clostridium botulinum toxoid type A, mouse IgM monoclonal antibody (US Biological, Swampscott, MA). Brown staining was considered an evidence for BTX-A presence. This study showed that BTX-A/ EMDA resulted in deeper and more homogeneous distribution of BTX-A into the rabbit bladder wall including urothelium, lamina propria and muscularis when compared with intravesical BTX-A injection.

In electromotive saline administration group, the urothelium, interstitium, and muscular layers were negative for staining. The findings of this study should be interpreted with caution and within the context of several limitations. First, precise laboratory investigations are needed to evaluate the stability of BTX-A's molecular structure under the influence of an electrical field. Secondly, the possibility of systemic drug absorption should be kept in mind, because BTX-A/EMDA resulted in deep drug penetration.

Thirdly, this study failed to show a physiological response of the BTX as the authors did not perform any urodynamic studies. 
Finally, recruitment of a control group in which the toxin is intavesically instilled without any further intervention would probably help in better understanding of the effect of electrical field in deep penetration of the toxin (81). Nevertheless, this technique has potential for clinical use translation but requires further investigation. The cost of the equipment and need to come to a medical facility to get the procedure done is a limitation.

\subsubsection{Intravesical BTX-A instillation using low-energy shock wave therapy:}

In another study on female Sprague Dawley rats, low-energy shock waves (LESW) were used to enhance urothelial permeability prior to drug instillation. Rats were anaesthetized, baseline urodynamic study was conducted at baseline, and then either saline or BTX-A were administered by intravesical instillation. On day \#8, rats were again anaesthetized and another urodynamic study was performed prior to bladder harvesting and immunohistochemical staining for SNAP-23, SNAP-25 and COX2. Magnetic resonance imaging (MRI) was also performed after intravesical administration of Gddiethylenetriamine pentaacetic acid (GPA) to document any urothelium leakage after LESW.

Pre-treatment with LESW was documented to increase urothelial permeability and facilitate intravesical BTX-A delivery, as indicated by decreased SNAP-25, SNAP-23 and COX2 expression in LESW pre-treated versus non-pre-treated rats. Urodynamic findings suggested blockage of the acetic acid-induced bladder overactivity in LESW pre-treated rats. The authors concluded that LESW was a novel and promising technique that would require further investigation. Though still not definitively proven method by which to deliver BTX-A without the need for injections (83).

\subsubsection{Intravesical BTX-A instillation using Protamine Sulphate:}

In this study the effect of intravesical BTX-A administration on Calcitonin Gene Related Peptide (CGRP) immunoreactivity and bladder hyperactivity was investigated in an acetic acid-induced bladder pain model in rats. 
Experimental and control animals were catheterized and intravesically exposed to protamine sulfate $(1 \mathrm{ml}, 10 \mathrm{mg} / \mathrm{ml})$, followed by BTX-A $(1 \mathrm{ml}, 25 \mathrm{U} / \mathrm{ml})$ or saline, respectively. 3 and 7 days after intravesical therapy continuous cystometrograms were performed under urethane anesthesia by filling the bladder $(0.08 \mathrm{ml}$ per minute $)$ with saline, followed by $0.3 \%$ acetic acid instillation. Bladder immunohistochemistry was used to detect Calcitonin Gene Related Peptide (CGRP) (84).

The author found that the inter-contraction interval (ICI) was decreased after acetic acid instillation $(50.2 \%$ and $65.0 \%$ ) in the control group at days 3 and 7 , respectively. However, rats that received BTX-A showed a significantly decreased response $(28.6 \%$ ICI decrease) to acetic acid instillation at day 7. This effect was not observed at day 3 (62.2\% ICI decrease). On other words, the ICI values were almost the same between control and BTX-A treated groups at day 3 meanwhile they start to show significant difference only at day 7. Increased CGRP immunoreactivity was detected in the BTX treated group at day 7 but not at day 3 , meanwhile it was detected in the control group both at day 3 and 7 .

The authors concluded that; intravesical BTX using Protamine Sulphate blocked acetic acid- induced bladder pain response and inhibits CGRP release from afferent nerve terminals. This study lacks certainty as the investigator was not blinded to analysis. On the other hand, a control group where only BTX-A-saline is instilled without protamine sulphate should be available. Parsons et al (1990) also reported that the intravesical treatment of rabbit bladder with protamine sulfate increased urothelial permeability, both in vivo and in vitro, to water, urea, and calcium (85). This effect was reversed with pentosan polysulfate. The authors concluded that the protamine sulfate somehow interfered with or damaged the GAG layer and that this was repaired by pentosan polysulfate (84). 


\subsubsection{Intravesical BTX-A instillation using Dimethyl Sulfoxide (DMSO)}

A phase I/II trial was recently performed at the Mayo Clinic in Rochester, Minnesota to evaluate the safety and potential efficacy of the intravesical instillation of a BTX-ADMSO solution to treat idiopathic DO in women (86).

Twenty-five women with medication-resistant, urodynamically-confirmed idiopathic DO were enrolled. Of these 25 patients, 22 received the full 300 units of botulinum toxin-A and $50 \mathrm{ml}$ of DMSO (50\% concentration), while three were given $66 \%$ of the full BTX-A dose.

Questionnaires assessing symptoms were completed at baseline and both one month and three months' post treatment. The primary outcomes of interest were adverse effects, number of episodes of incontinence, quantity of post-void residuals, and scores on the Blaivas-Groutz Anti-Incontinence scale, Indevus Urgency Severity Scale, Incontinence Impact Questionnaire, and Urogenital Distress Inventory. One- and three-month scores were compared against baseline values.

At the end of three months of observation, no serious adverse events were reported. Clinically-important increases in post-void residual were reported, the median number of incontinent episodes had decreased, and an overall improved quality of life was noted. Randomized, double-blind, placebo-controlled trials are needed to verify these results.

\subsection{Use of hyaluronan-phosphatidylethanolamine (HA- $\mathrm{PE}$ ) to enhance BTX-A delivery}

The term "hyaluronan" was introduced in 1986 to describe a ubiquitous extracellular matrix tissue polysaccharide that belongs to the glycosaminoglycan (GAG) family. It is characterized by repeating hexosamines and uronic acid (87-89). Its functions in the body are, amongst other things, to bind water and lubricate movable parts of the body, like joints and muscles. At Western University, London, Canada, a non-particulate formulation was developed that increases the penetration of HA through the epidermis. 
It forms HA coats around epidermal cells and takes advantage of HA polymers that permit the encapsulation of large proteins like BTX-A and their more efficient delivery to the dermis. This was achieved by linking HA to phospholipid chains and selecting those formulations that promote HA coat formation around keratinocytes. The high viscosity of these formulations allows it to enmesh large proteins like BTX- A, which can then be transported with HA-PE into the epidermal layer.

An experimental study within Western University's Department of Plastic Surgery was recently conducted using this newly-formulated HA-PE. In this study, forty-five 10month old, retired breeder female C57BL/6 mice (Jackson Laboratory) were used for multiple cream application studies (26), while an additional twelve 12-month old retired breeder female C57BL/6 mice were used for Alexa647 mouse experiments. For this, all the mice were anesthetized prior to cream application. While anesthetized, the upper dorsum of their back was shaved, leaving a strip of hair in the midline to define the right and left sides. Alexa647-HA $0.18 \mathrm{~g}$ and Alexa647-HA-PE cream were then applied to the left and right sides, respectively. Two mice were euthanized using $\mathrm{CO} 2$ chamber at each of six time points: $40 \mathrm{~min}, 2 \mathrm{~h}, 4 \mathrm{~h}, 8 \mathrm{~h}, 24 \mathrm{~h}$, and 72 hours following cream application. It was found that multiple topical applications of HA-PE to shaved mouse skin significantly stimulated basal keratinocyte proliferation and epidermal thickness, relative to HA or the vehicle cream alone. HA-PE had no detectable effect on keratinocyte differentiation nor caused local inflammation.

These effects of HA-PE polymers are similar to those reported for endogenous epidermal HA in youthful skin and show that the topical application of HA-PE polymers can restore some of the impaired functions of aged epidermis. Due to the successful penetration of HA-PE through the epidermal skin layers, we then proposed that HA-PE might penetrate through the urothelium, carrying large molecules like BTX-A along with it. It was this conjecture that led to the planning and orchestration of the current research project. 


\subsection{Anatomy of the rat bladder}

The wall of the rat urinary bladder is made up of three layers namely, mucosa, muscularis and adventitia. The mucosa is thrown into numerous folds and consisted of a lining urinary epithelium and a fibroelastic lamina propria. The lining epithelium is transitional consisting of basal, intermediate and superficial cells. The basal cells were mainly cuboidal in form with central nuclei. The intermediate cells are spindle-shaped and arranged with their long axes perpendicular to the basal lamina. The superficial cells are large and dome-shaped with abundant eosinophilic cytoplasm.

The luminal border also showed deep invaginations giving the surface a characteristic scalloped appearance. The lamina propria consisted mainly of collagenous connective tissue containing small blood vessels (90).

The muscularis appeared to be formed of three interlaced layers; inner and outer thin longitudinal layers and a middle thick layer. As a result of intermingling of muscle fibers in these layers, the muscularis cannot be clearly separated into three well-defined strata. The muscle fibers are arranged into groups separated by loose connective tissue septa. The structure of the rat bladder urothelium is very similar to human urothelium as regard the type of epithelium and the number of cell layers that makes it very suitable to perform several experimental studies.

On the other hand, mice bladders are very tiny compared to rat bladders that makes rat bladders more applicable to research purposes than the tiny mouse bladder. Female rats are more preferable to male rats when research on the rat bladder is desirable because the female rat urethra is shorter and urethral catheterization, is more feasible. 

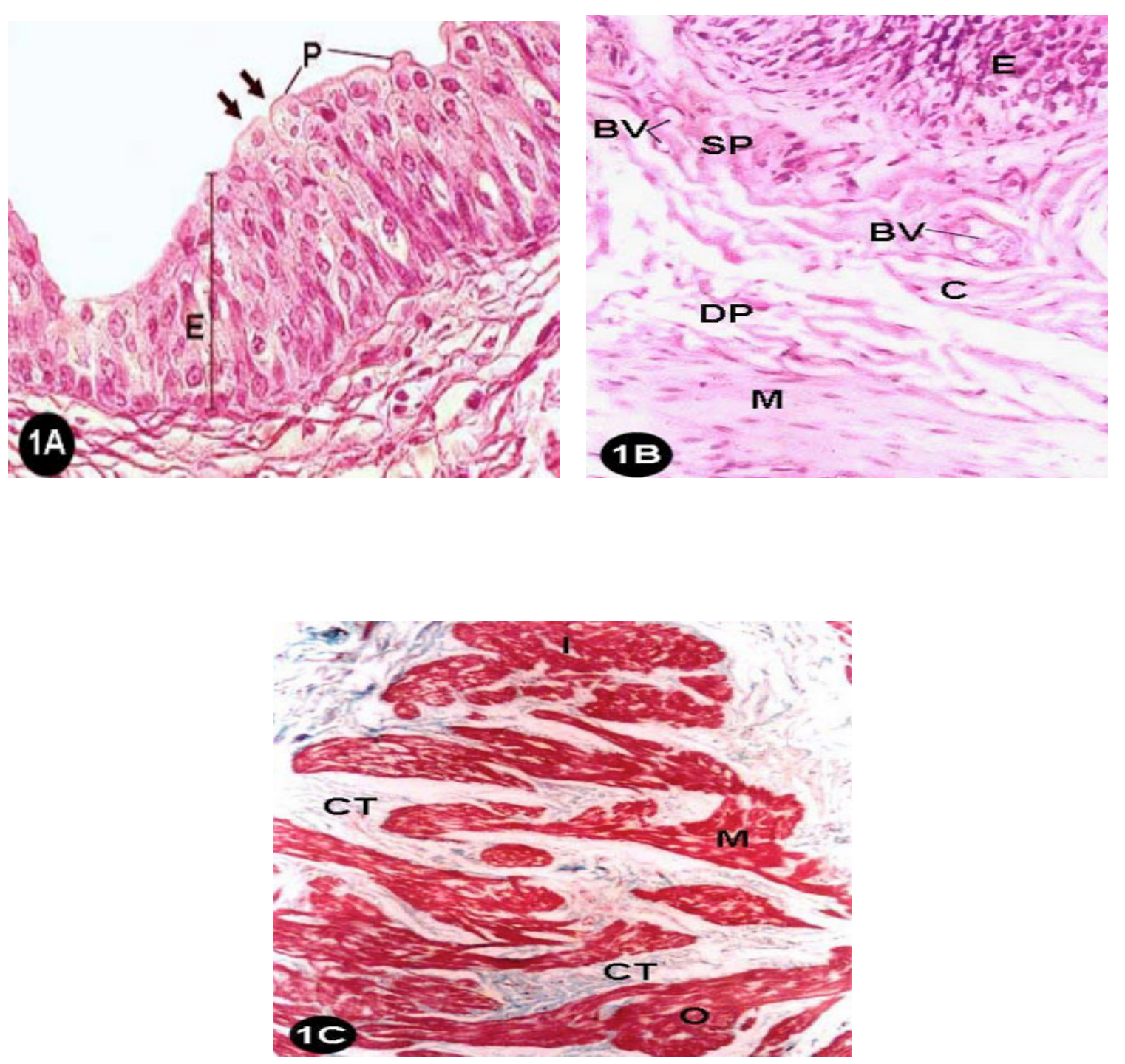

Figure 6: Photomicrographs of sections of the urinary bladder of adult female rats showing the bladder mucosa (1A). Lamina propria (1B) and muscularis mucosa (1C). Int. J. Morphol. 2005, vol.23, n.4, pp.309-316. ISSN 0717-9502. 


\section{Chapter 3: Methods}

This study is an experimental, pilot study in a rat model to assess the feasibility and early results of using a novel carrier hyaluronan-phosphatidylethanolamine (HA-PE) to aid botulinum toxin A (BTX-A) delivery to the bladder muscle using bladder instillation instead of direct bladder muscle injection, our current standard clinical practice.

\subsection{Objective and outcomes measured}

\section{a) Primary objective and outcome}

The primary objective of this study was to demonstrate the action of hyaluronanphosphatidyl-ethanolamine (HA-PE)- BTX-A bladder instillation on SNAP-25 cleavage and compare it to SNAP-25 cleavage achieved by direct bladder muscle injection of BTX-A. This outcome was measured by estimating the mean SNAP-25 staining percentage using immuno-histochemistry in the corresponding tissue slides.

\section{b) Secondary objectives and outcomes}

1) Assess our surgical technique and standardize the urodynamic study protocol.

2) To demonstrate the translocation of Alexa Fluor ${ }^{\circledR} 594$-labelled BTX-A across the urothelium using fluorescence under the confocal microscope.

3) To demonstrate the physiological actions of the translocated BTX-A-HA-PE on urodynamic parameters (maximum pressure and inter-contraction intervals) compared to direct bladder muscle injection of BTX-A, after acetic acid instillation.

4) Assess the effect of HA-PE dose and duration of instillation on the efficiency of BTX-A delivery using HA-PE solution.

5) Assess the toxicity and/or inflammatory changes caused by the HA-PE carrier on the bladder urothelium and musculature using histological assessment. 


\subsection{Study Design}

This experimental study was conducted on sixty female Sprague Dawley rats (250-350 g), which were equally divided (10 rats per group) into three control groups and three treatment groups, (Fig.7).

\section{a) Control groups}

Group \#1 rats $(\mathrm{n}=10)$ received bladder instillation of BTX-A-saline solution (10 $\mathrm{U}$ in 2 $\mathrm{ml}$ saline) for 60 minutes to assess whether a simple, presumably therapeutically-inert BTX-A- saline solution would be capable of crossing the urothelium and be physiologically effective. Group \#2 rats $(n=10)$ received bladder instillation of the HAPE carrier ( $0.5 \mathrm{~g}$ dissolved in $2 \mathrm{ml}$ saline) for 60 minutes. The purpose of this control group was to assess for possible HA-PE effect on bladder urothelium and musculature to rule out the inflammatory changes in the bladder. Group \#3 rats $(n=10)$ received BTX-A ( 5 units diluted in $0.2 \mathrm{ml}$ of saline) by direct injection into the external bladder surface into the lateral, anterior and posterior bladder walls performed through a midline laparotomy. This group served as a control to mimic the standard clinical BTX-A delivery through cystoscopic bladder muscle injection.

\section{b) Treatment groups}

Group \#4 rats $(\mathrm{n}=10)$ received bladder instillations of Alexa Fluor ${ }^{\circledR 594-L a b e l l e d ~ B T X-~}$ A $10 \mathrm{U}$ mixed with HA-PE $0.5 \mathrm{~g}$ in $2 \mathrm{ml}$ saline. Group \#5 rats $(\mathrm{n}=10)$ received bladder instillations of BTX-A 5U-HA-PE (in different doses, ranging from 0.2 to $0.5 \mathrm{~g}$ ) dissolved in $2 \mathrm{ml}$ of saline for 60 minutes. We further classified this group to sub-groups based on HA-PE dose; 5A where we used higher HA-PE doses $(0.4$ or $0.5 \mathrm{~g})$ and 5B where lower doses were used ( 0.2 or $0.3 \mathrm{~g})$ with identical instillation times of 60 minutes. Group \#6 rats $(\mathrm{n}=10)$ received bladder instillation of BTX-A 10 U-HA-PE $(0.2$ to $0.5 \mathrm{~g})$ dissolved in $2 \mathrm{ml}$ saline for 30 minutes. We further classified this group to sub-groups based on HAPE dose; 6A where we used higher HA-PE dose $(0.4$ or $0.5 \mathrm{~g})$ and $6 \mathrm{~B}$ where lower doses were used $(0.2$ or $0.3 \mathrm{~g})$ with identical instillation times of 30 minutes (Fig. 8\&9). 


\section{Female Sprague Dawley rats}

$$
\mathrm{N}=60
$$

\section{Control Groups}

$$
\mathrm{N}=30
$$
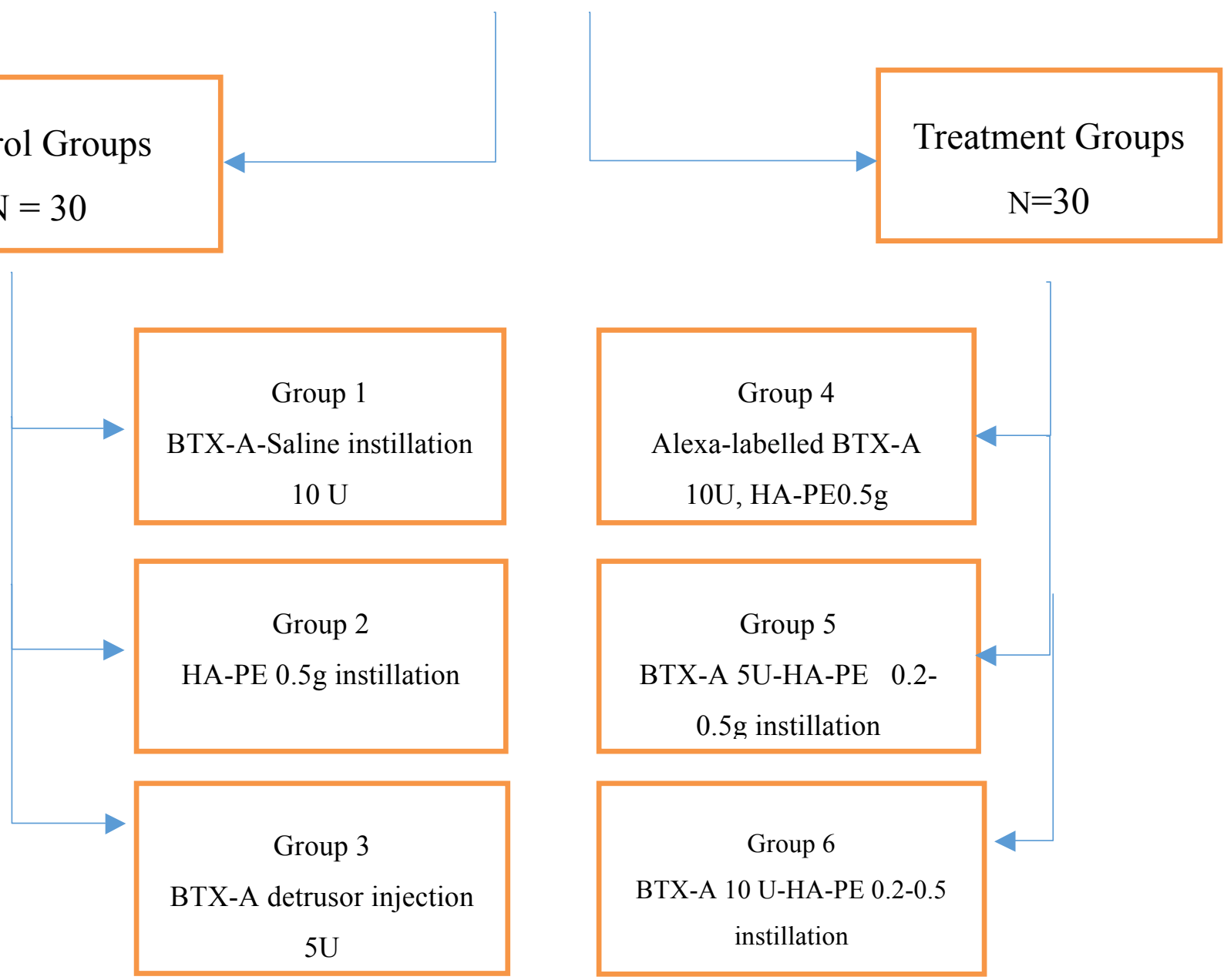

Fig. 7: Figure showing control groups 1-3 and treatment groups 4-6 


\begin{tabular}{|c|c|c|c|c|}
\hline 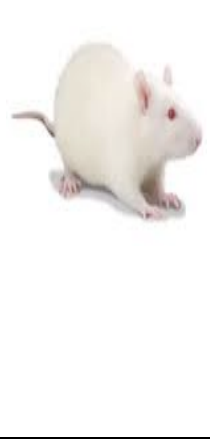 & $\begin{array}{l}\text { Group } 1 \\
\mathrm{~N}=10\end{array}$ & $\begin{array}{l}\text { Day } 0 \\
\text { Baseline } \\
\text { urodynamic } \\
\text { study }\end{array}$ & $\begin{array}{c}\text { Day 0 } \\
\text { BTX-A-saline } \\
10 \mathrm{U} \text { instillation } \\
60 \text { minutes }\end{array}$ & $\begin{array}{c}\text { Day } 14 \\
1 \% \text { Acetic acid } \\
\text { instillation followed by } \\
\text { urodynamic study, } \\
\text { tissue harvest and } \\
\text { euthanasia }\end{array}$ \\
\hline & $\begin{array}{l}\text { Group } 2 \\
\mathrm{~N}=10\end{array}$ & $\begin{array}{l}\text { Day 0 } \\
\text { Baseline } \\
\text { urodynamic } \\
\text { study }\end{array}$ & $\begin{array}{c}\text { Day } 0 \\
\text { HA-PE-saline } \\
0.5 \mathrm{~g} \text { instillation } \\
60 \text { minutes }\end{array}$ & $\begin{array}{c}\text { Day } 14 \\
1 \% \text { Acetic acid } \\
\text { instillation followed by } \\
\text { urodynamic study, } \\
\text { tissue harvest and } \\
\text { euthanasia }\end{array}$ \\
\hline$\stackrel{1}{1}$ & $\begin{array}{l}\text { Group } 3 \\
\mathrm{~N}=10\end{array}$ & $\begin{array}{l}\text { Day } 0 \\
\text { Baseline } \\
\text { urodynamic } \\
\text { study }\end{array}$ & $\begin{array}{l}\text { Day 0 } \\
\text { BTX-A 5U intra } \\
\text { detrusor injection }\end{array}$ & $\begin{array}{c}\text { Day } 14 \\
1 \% \text { Acetic acid } \\
\text { instillation followed by } \\
\text { urodynamic study, } \\
\text { tissue harvest and } \\
\text { euthanasia }\end{array}$ \\
\hline
\end{tabular}

Fig. 8: Botulinum Toxin A- HA-PE- saline intravesical instillation (Control Groups) 


\begin{tabular}{|c|c|c|c|c|}
\hline 4 & Group 4 & $\begin{array}{l}\text { Day } 0 \\
\text { Baseline } \\
\text { urodynamic } \\
\text { study }\end{array}$ & $\begin{array}{c}\text { Day 0 } \\
\text { Alexa }{ }^{\circledR 594-B T X-10} \\
\text { U HA-PE 0.5g-saline } \\
\text { instillation } \\
60 \text { minutes }\end{array}$ & $\begin{array}{c}\text { Day } 14 \\
1 \% \text { Acetic acid } \\
\text { instillation followed by } \\
\text { urodynamic study, tissue } \\
\text { harvest and euthanasia }\end{array}$ \\
\hline & $\begin{array}{c}\text { Group } \\
5 A \\
N=4 \\
\text { Group } \\
5 B \\
N=6\end{array}$ & $\begin{array}{l}\text { Day 0 } \\
\text { Baseline } \\
\text { urodynamic } \\
\text { study }\end{array}$ & $\begin{array}{l}\text { BTX-A } 5 \mathrm{U} / \mathrm{HA}-\mathrm{PE} \\
0.4 \text { or } 0.5 \mathrm{~g} \text {-saline } \\
\text { instillation } \\
60 \text { minutes }\end{array}$ & $\begin{array}{c}\text { Day } 14 \\
1 \% \text { Acetic acid } \\
\text { instillation followed by } \\
\text { urodynamic study, tissue } \\
\text { harvest and euthanasia }\end{array}$ \\
\hline-4 & $\begin{array}{c}\text { Group } \\
6 A \\
N=5 \\
\text { Group } \\
5 B \\
N=5\end{array}$ & $\begin{array}{l}\text { Day } 0 \\
\text { Baseline } \\
\text { urodynamic } \\
\text { study }\end{array}$ & $\begin{array}{c}\text { Day 0 } \\
\text { BTX-A } 10 \mathrm{U} / \mathrm{HA}-\mathrm{PE} \\
0.4 \text { or } 0.5 \mathrm{~g} \text {-saline } \\
\text { instillation } 30 \\
\text { minutes }\end{array}$ & $\begin{array}{c}\text { Day } 14 \\
1 \% \text { Acetic acid } \\
\text { instillation followed by } \\
\text { urodynamic study, tissue } \\
\text { harvest and euthanasia }\end{array}$ \\
\hline
\end{tabular}

Fig. 9: Botulinum Toxin A- HA-PE- saline intravesical instillation

\section{(Treatment Groups)}


All rats in the control and treatment groups underwent the identical anesthetic and surgical procedures on Day 0, accompanied by a baseline urodynamic study. A second procedure was performed on all rats on Day \#14. This was followed by an urodynamic study, after $1 \%$ acetic acid instillation for 30 minutes (Fig.8\&9). Rats were subsequently euthanized and their bladders were harvested, as detailed below.

\subsection{Pre-operative care}

\section{Rat housing}

Rats were housed in the animal lab facility at the Lawson Research Institute, St. Joseph's Hospital, Western University, London, Canada. All rats were housed in conventional rat cages, two rats per cage, in a temperature-controlled environment with a 12-hour light/dark cycle. Rats were fed a standard rat chow diet and observed for 3 days in the animal lab facility for acclimatization before their enrollment into the study.

\subsection{Pre-operative preparation}

\subsubsection{BTX-A reconstitution}

BTX-A was reconstituted by adding $2 \mathrm{ml}$ of normal saline to a $50 \mathrm{U}$ vial, generating a concentration of $5 \mathrm{U} / 0.2 \mathrm{ml}$. Reconstituted BTX-A was kept in a $4{ }^{\circ} \mathrm{C}$ fridge in the animal lab facility and was used within 14 days of the reconstitution date to maintain its effectiveness.

\subsubsection{HA-PE liquefaction}

HA-PE is a yellowish viscous paste like substance, Fig 10. There was no pre-defined standard dose of HA-PE for BTX-A delivery to be liquefied adequately in $2 \mathrm{ml}$ of saline (the average rat bladder volume) nor any previous study to assess the degree of viscosity achieved after dissolving HA-PE in saline, which would allow injection through a $6 \mathrm{~mm} / 28$-gauge needle for bladder instillations. 
Therefore, we used different doses of HA-PE to assess both adequate liquefaction as well as the impact of HA-PE dose on BTX-A delivery.

One of our secondary aims was to define the highest possible HA-PE dose that could be sufficiently liquefied in $2 \mathrm{ml}$ of saline for injection through 28 -gauge needle. We started with the highest possible soluble HA-PE weight $(0.5 \mathrm{~g})$ that could be liquefied in $2 \mathrm{ml}$ saline and then reduced the dose in $0.1 \mathrm{~g}(0.4,0.3,0.2 \mathrm{~g})$. The appropriate dose of BTX-A was then added to the HA-PE solutions, in accordance with assigned group specifications.
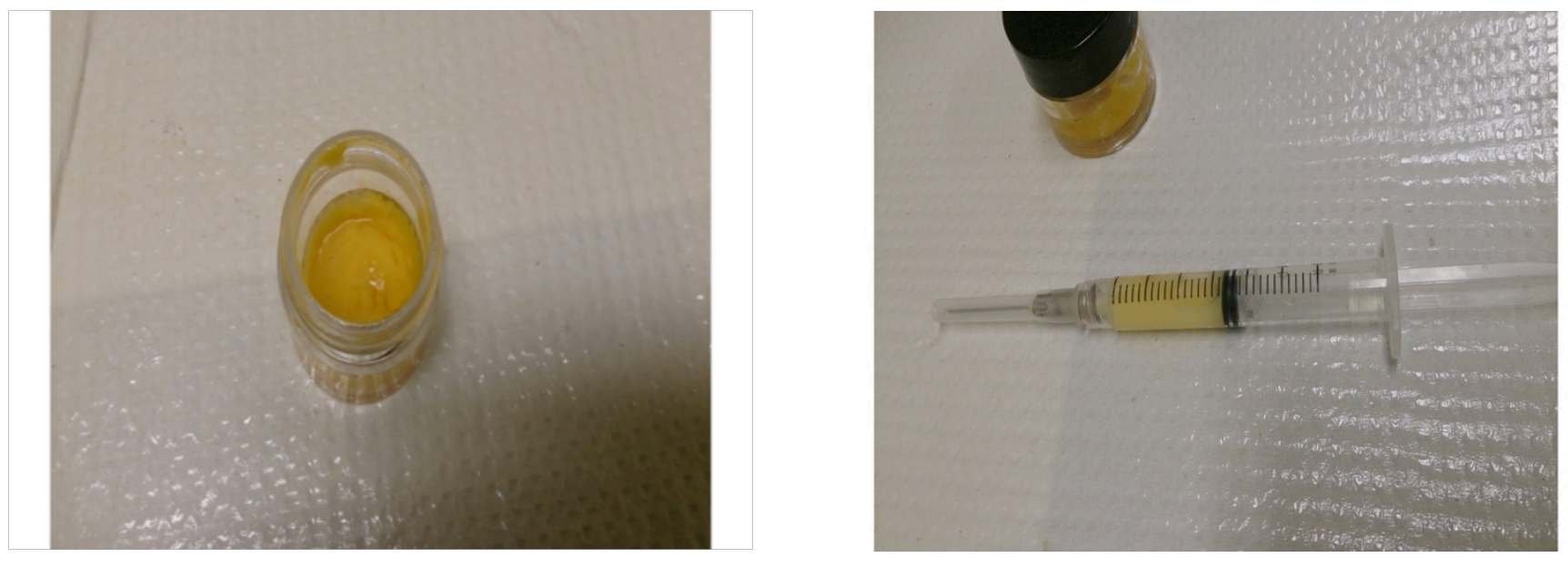

Fig. 10: HA-PE in its original form as a paste (Left) and after liquefaction in saline (Right)

\subsubsection{Alexa ${ }^{\circledR 594-~ B T X-A ~ l a b e l i n g ~}$}

Labeling of BTX-A with Alexa Fluor ${ }^{\circledR} 594$ was carried out at Robarts Research Institute, Western University, Canada, using a standardized protocol, appendix 3. 


\section{Supplies:}

Life Technologies (Thermo- Fischer Scientific) Cat A20004 Alexa Fluor® 594 NHS Ester, $1.3 \mathrm{mg}$ (succinimidyl ester). Dialysis Tubing - MWCO 6000 -8000 - Spectrum Labs Cat 132660.

\section{Solutions used:}

Sodium bicarbonate $9.0 \mathrm{pH}$ solution, Alexa solution was prepared using $1.3 \mathrm{mg}$ Alexa Fluor ${ }^{\circledR 594}$ and Dialysis Solution for BTX-A dialysis, 4L of 1*PBS (Phosphate Buffered Saline).

\section{Steps:}

BTX-A was put in dialysis tubing immersed in Alexa Fluor ${ }^{\circledR 594}$ solution and was left for 24 hours at $4{ }^{\circ} \mathrm{C}$ in the dark (wrapped in foil). Then, we dialyzed BTX-A in $1 *$ PBS for 2-3 days with change in the buffer every 6-12 hours (6 changes).

\subsection{Anesthetic procedure}

All rats were weighed and checked for activity prior to the initial procedure (day zero). The standard anesthetic procedure was used, the rat was placed in the glass anesthesia chamber, (Fig.11), and Isoflurane inhalation at an induction dose of $4 \mathrm{ml} / \mathrm{min}$ was used, until the rat was completely anesthetized. Once anesthetized, the rats were taken out of the glass chamber and received Isoflurane through the rat mask (Fig.12) at a maintenance dose of $2 \mathrm{ml} / \mathrm{min}$ throughout the entire surgical procedure. 


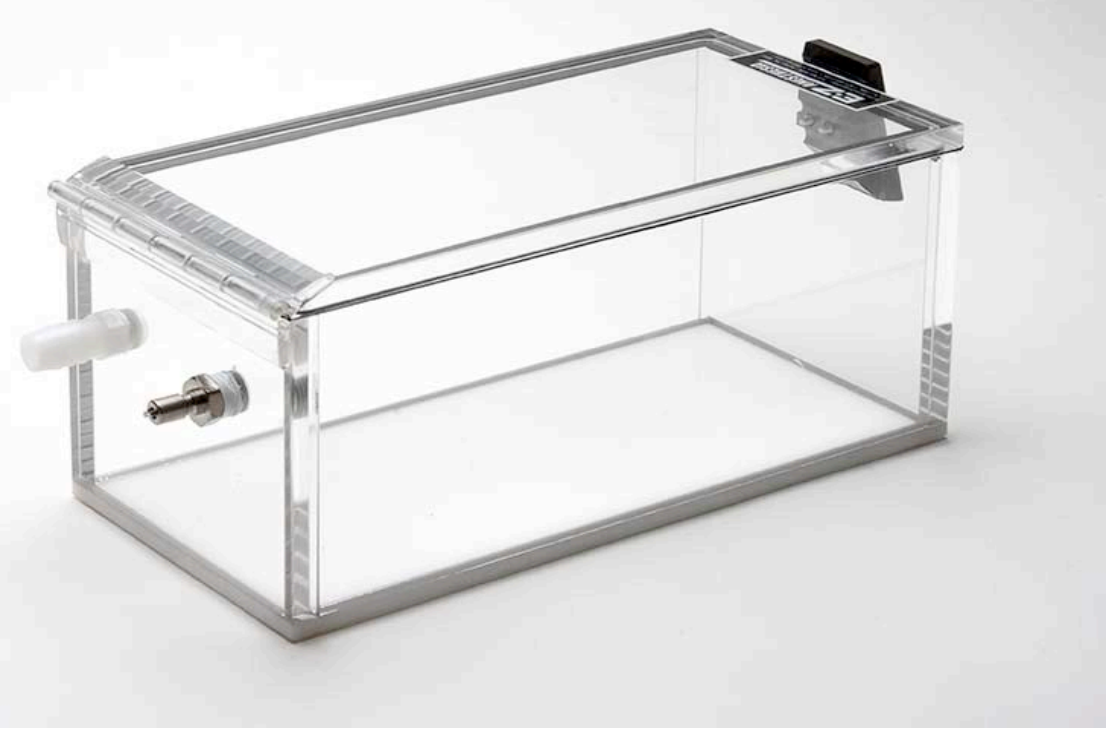

Fig. 11: Glass chamber for gas anesthesia

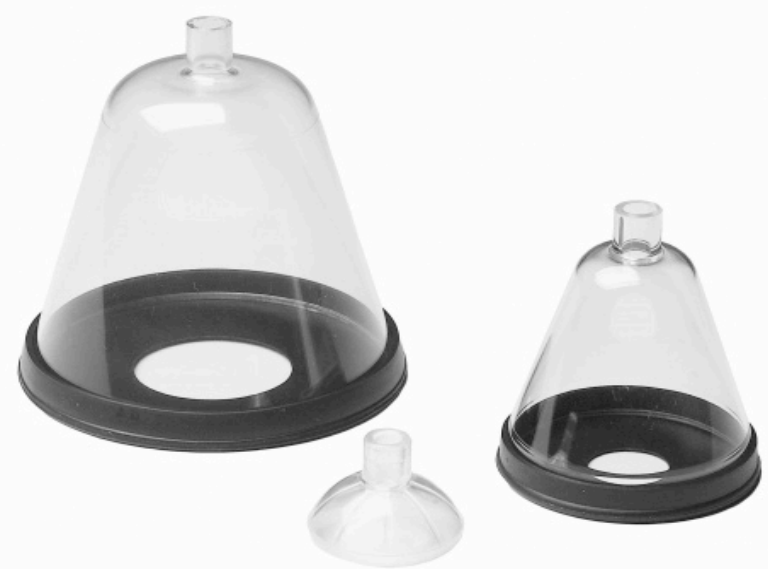

Fig. 12: Rat mask 


\subsection{Surgery procedure and postoperative care 3.6.1. Initial surgery on day zero}

For the initial surgery, the animal was positioned supine with the animal's abdomen facing up. The lower part of the abdomen was shaved with an electric clipper, after which a standard 3-step sequential sterilization procedure was performed, using chlorhexidine, alcohol and betadine, in order. A lower midline abdominal incision $(1-2 \mathrm{~cm})$ was then created and the bladder identified. A urodynamic assessment was performed, as discussed later.

Group \#1 rats: The pre-prepared mixture of BTX-A 10U in $2 \mathrm{ml}$ saline was instilled into the bladder by direct bladder puncture. Care was taken to avoid rapid instillation and prevent leakage through the urethra. The instilled mixture was retained in the bladder for 60 minutes.

Group \#2 rats: The pre-prepared mixture of HA-PE $0.5 \mathrm{~g}$ dissolved in $2 \mathrm{ml}$ saline was instilled gradually into the bladder by direct bladder puncture and retained in the bladder for 60 minutes.

Group \#3 rats: BTX-A ( $5 \mathrm{U}$ in $0.2 \mathrm{ml}$ saline) intra-detrusor injection was achieved by direct injection into the lateral, anterior and posterior walls of the bladder at 4-5 sites from the peritoneal surface of the bladder. Raised blebs were considered as an adequate sign of successful injection into the detrusor muscle.

Group 4 rats: $2-3 \mathrm{ml}$ of a pre-prepared mixture of Alexa Fluor®594- Labeled BTX-A 10U-HA-PE $0.5 \mathrm{~g}$ in $2 \mathrm{ml}$ saline were instilled gradually into the bladder by direct bladder puncture. The mixture was retained in the bladder for 60 minutes. Care was taken to avoid direct exposure of the mixture to light by covering the syringe with opaque tape and covering the bladder after instillation with moist towel during the entire instillation.

Group \#5 rats: 2-3 ml of the pre-prepared mixture of BTX-A 5U-HA-PE (dose varied between $0.2-0.5$ grams) in saline was instilled gradually into the bladder by direct bladder puncture. The instilled mixture was retained in the bladder for 60 minutes. 
Group \#6 rats: 2-3 ml pre-prepared mixture of BTX-A10U-HA-PE (dose varied from 0.20.5 grams) was instilled gradually into the bladder by direct bladder puncture. The mixture was retained in the bladder for 30 minutes.

After instillation for either 30 or 60 minutes, as per group assignment, the bladder was drained by applying gentle manual pressure on the bladder dome to evacuate the solution per urethra.

Supplemental heat was provided by a heat lamp and a pre-heated pad that had been autoclaved for 30 seconds prior to commencing the procedure. During the entire duration of surgery, adequate hydration via intra-peritoneal saline injection was used to compensate for unavoidable fluid losses, and the surgical wound was covered with a wet gauze. Respiratory movements and neurological reflexes were monitored throughout the procedure. Rectal temperature was monitored using a baby thermometer lubricated with $\mathrm{K}-\mathrm{Y}$ gel. The bladder was then closed in a water tight manner using 7-0 PDS. Abdominal muscles were closed with interrupted Vicryl 3-0 suture in one layer followed by subcuticular 3-0 Vicryl sutures for the skin closure.

\subsubsection{Final surgery and euthanasia on day 14}

After two weeks, rats were re-anesthetized using the previously described protocol. For 30 minutes, $2 \mathrm{ml}$ of $1 \%$ acetic acid were administered by direct bladder puncture using a 6 $\mathrm{mm} / 28$-gauge needle. K-Y Jelly was applied to the external urinary meatus to prevent leakage through the urethra. Acetic acid was then drained through the urethra.

Urodynamic assessment was carried out as described later (Section 3.7.2.2). The rat was then euthanized by exsanguination (transecting the aorta) and the urinary bladder was harvested.

In Group \#4 rats (10 rats), in which Alexa Fluor®594-labelled BTX-A was used, the common iliac artery was ligated and saline was injected into the internal iliac artery distal to the ligature to washout as much blood as possible from the harvested bladder tissue, so as to minimize any auto-fluorescence of blood when analyzing Alexa Fluor®594 red fluorescence thereafter. 


\subsubsection{Post-operative care}

Rats who received BTX-A, either via instillation or through intra-detrusor injection, were housed in filter-top cages, two rats per cage, for the first 24 hours of the 2-week experimental protocol (days 0 to 14). Subsequently, they were transferred to conventional rat cages until the date of the second surgical procedure and ultimate euthanasia.

Rats in Group \#2, in which HA-PE-saline was instilled, were moved to conventional rat cages immediately after recovery and maintained there until the second procedure and ultimate euthanasia.

Rats were observed post-operatively twice daily for the first three days and once per day thereafter until they were euthanized. On each of the first three post-operative days, rats were weighed and observed for the following: activity; urine excretion; stool production; and certain body postures, like hunching, which is suggestive of pain or infection. All pre- and post-operative observational data was registered on pre-prepared animal observation sheets. If an infection was suspected, the rat was euthanized, as per protocol.

\subsection{Outcomes assessment}

\section{Tissue processing:}

All bladder specimens were fixed in 4\% paraformaldehyde at $4 \circ \mathrm{C}$ for 24 hours. Specimens were processed for SNAP-25 staining as paraffin sections at the London Regional Cancer Program (LRCP) facility in London, Ontario. An automated tissue processor - Leica ASP300 — was used to process the tissues. The microtome used for cutting was a Leica Rm2255 and the samples were cut at a thickness of $5 \mu \mathrm{m}$. Group \#4 (Alexa Fluor®594 -labelled BTX-A) specimens were processed as frozen sections and prepared for examination using the confocal microscope at St Joseph's Hospital in London, Ontario. Group \#1 controls, which received BTX-A10U-saline instillation without were processed similarly and used as negative controls for group 4 . The cryostat used for cutting was a Leica CM3050S, and samples were cut at a thickness of $10 \mu \mathrm{m}$. 


\subsubsection{Primary outcome assessment}

\section{SNAP-25 staining}

Day 1

The first step was deparaffinization of the slides by immersing them in Xylene for 15 minutes then alcohol series (100-95-70 \%) each for 10 minutes followed by rehydration using distilled water and 1*PBS (Phosphate -buffered saline) for 5 minutes each. Antigen retrieval done by immersion of tissue slides in Sodium Citrate, $\mathrm{pH}$ 6.0.and microwave it at different temperature levels. Then, slides were immersed in $3.0 \% \mathrm{H} 2 \mathrm{O} 2$ diluted in 1 PBS for 10 minutes. Antigen block with 3.0\% BSA (Bovine Serum Albumin) diluted in 1 BPS then took place for 1-2 hours. Primary antibody (sab 2500965, Sigma Aldrich, Canada) was then added to the slides and tissue slides were left covered in tinfoil overnight in cold fridge at $\left(4^{\circ} \mathrm{C}\right)$. SNAP- 25 antibodies are supplied at $0.5 \mathrm{mg} / \mathrm{mL}$ in saline with $0.02 \%$ sodium azide and $0.5 \%$ bovine serum albumin.

Day 2

Secondary antibody (anti-rabbit IgG-goat) at a dilution of $1 \mathrm{ul}$ added to $500 \mathrm{ul}$ of 1 *PBS was added to the slides. Streptavidin at a dilution of 1:2000 ul $1 *$ PBS was added to the slides and left in room temperature for 30 minutes. DAB has been added and the slides and left from 5 minutes to 40 minutes according to the evidence of reaction. Sections were immersed in Haematoxylin for 30 seconds then washing in distilled water for 5 minutes followed. Tissue slides were then run through original series in reverse (70\%95\%-100\%) Ethanol each is 2 minutes' duration and Xylene for 1 minute at the end. The slides were mounted in Richard-Allan Scientific Cytoseal 60 (Thermo-Scientific). Mounted slides were left to dry overnight in the lab. 


\section{SNAP-25 Examination}

Tissue slides were scanned at the LRCP facility using the Aperio Scan Scope. Several pictures (4 pictures) were captured randomly for each slide, from different slide corners. The 4 captured pictures from each slide were then analyzed quantitatively using Image $\mathbf{J}$ software, and the picture which showed the highest staining density was selected to represent the SNAP-25 staining percentage of this slide.

Using image $\mathrm{J}$ software, the same settings were applied to all tissue slides to obtain a SNAP-25 staining score, which was then divided by the total slide area score to calculate the percentage of SNAP-25 staining. Bladder urothelium was also examined for evidence of HA-PE-induced toxicity and inflammation, denoted by the presence of inflammatory cells and the level of integrity of cell lines. Functional activity of BTX-A using SNAP-25 staining shows a brown colour when the molecule is intact and absence of brown colour when it is cleaved. Lack or scarcity of brown colour in the tissue slides denoted SNAP-25 cleavage and was used as a proof of BTX-A activity in bladder muscle. Conversely, abundance of brown colour denoted that SNAP-25 molecules were intact (not cleaved), arguing against any significant BTX-A activity.

\subsubsection{Secondary outcomes assessment}

\subsubsection{Alexa Fluor ${ }^{\circledR 594-l a b e l l e d ~ B T X-A ~ f l u o r e s c e n c e ~}$}

Experimental group 4 and control group 1 frozen tissue slides were stained with DAPI (4', 6-diamidino-2-phenyl-indole ) to stain tissue nuclei and examined for Alexa Fluor®594 red fluorescence under the Olympus Confocal Microscope at St Joseph’s Hospital, London. Canada. Slide examination under the confocal microscope was done by an experienced blinded scientist who was not a member of the study team.

\subsubsection{Urodynamic assessment}

The urodynamic study was performed under anesthesia at baseline and after 2 weeks of the initial procedure. The second study was performed after $1 \%$ acetic acid intravesical instillation. 
Both studies were performed identically using slow fill saline $(0.1 \mathrm{ml} /$ minute as per the protocol), a 26-gauge bladder angiocath representing the saline input arm was connected to one side of a 2-stopcock pressure transducer. The other side of the pressure transducer was connected to a micro-infusion pump that infused saline at a rate of $0.1 \mathrm{ml} /$ minute (Fig. 13).

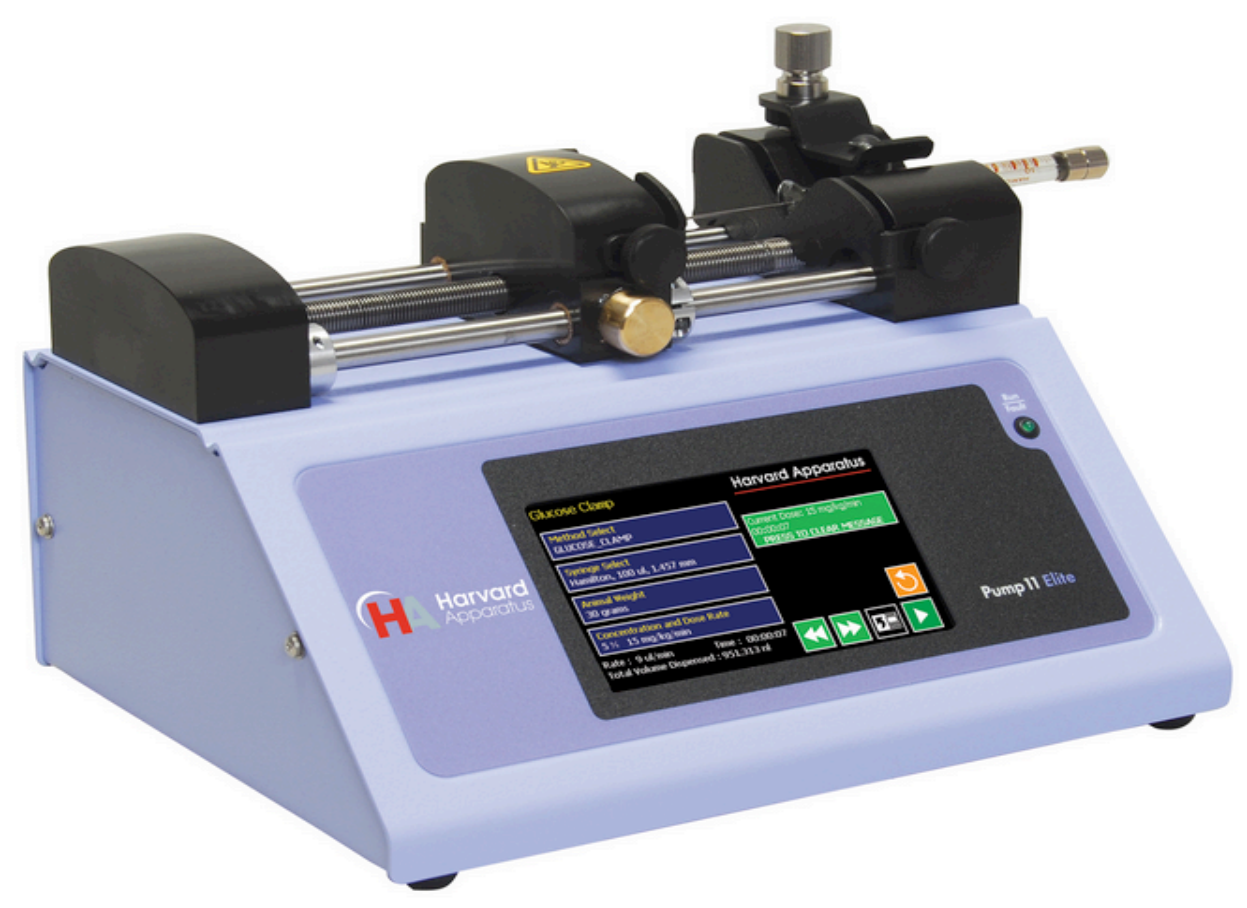

Fig. 13: Standard Infusion Only, 11 Elite Syringe Pump

A PE-50 tube representing saline output was connected to a micro-pressure analyzer that transformed the pressure inside the bladder into pressure waves and values which were digitally recorded, (Fig.14). 


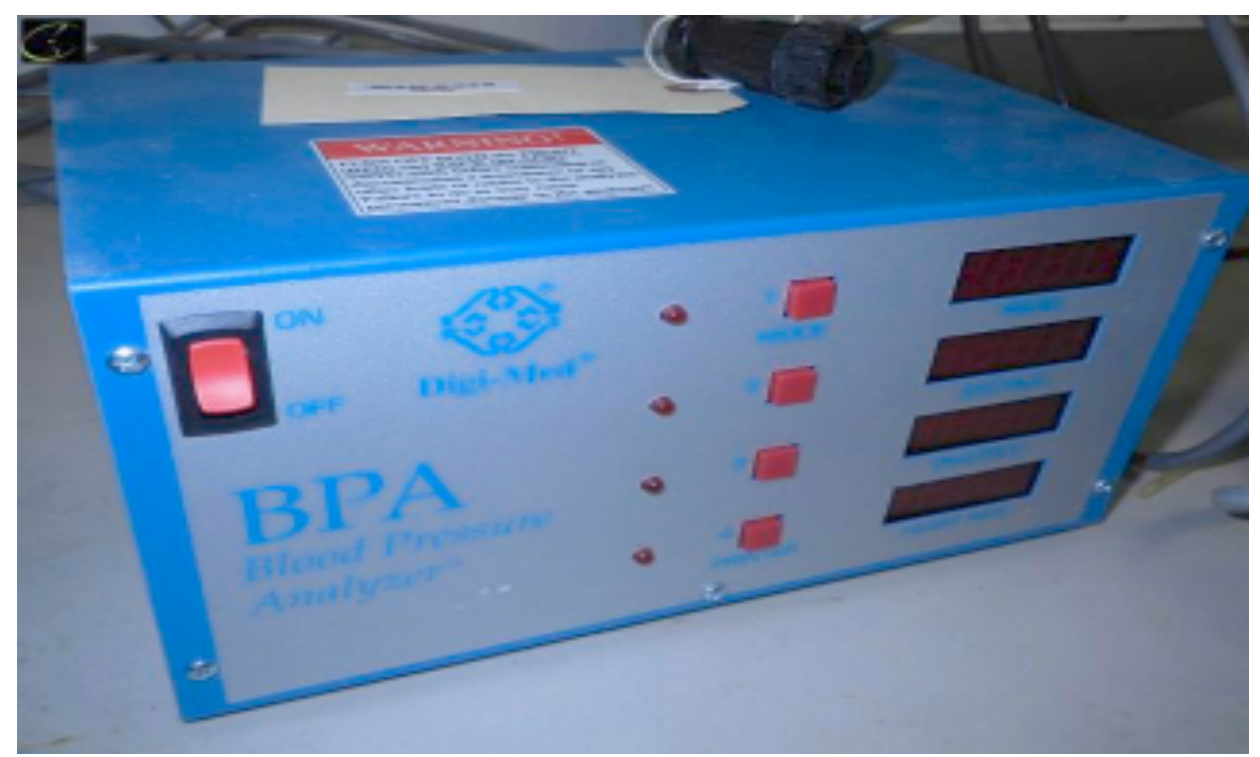

Fig.14: Digi-Med, Micro-Pressure Analyzer

All pressure values were automatically saved to each rat experiment on Digi-Med software. $2 \mathrm{ml}$ of normal saline were infused which represents the average rat bladder capacity of $250-350 \mathrm{~g}$ female rat.

Each urodynamic assessment measured two main parameters, which were:

Maximum Pressure (MP): The highest intravesical pressure recorded during bladder filling expressed as $\mathrm{mmHg}$.

Inter-Contraction Intervals (ICI): the average duration (milliseconds) between bladder contractions during bladder filling. It is calculated by measuring the time interval between the beginning of the first contraction to the beginning of the last contraction and then dividing by the number of inter-contraction intervals. In cases where there are multiple contractions, the number of the inter- contraction intervals is $n-1$, where $n$ is the number of contractions, e.g.; an urodynamic study with 4 contractions has 3 intercontraction intervals. 
In the example below; the first contraction starts at 11 milliseconds while the last contraction starts at 49 milliseconds. Hence, the total ICI is 38 milliseconds provided that we have 5 contractions and 4 inter-contraction intervals so the average ICI is $38 / 4=9.5$ milliseconds.

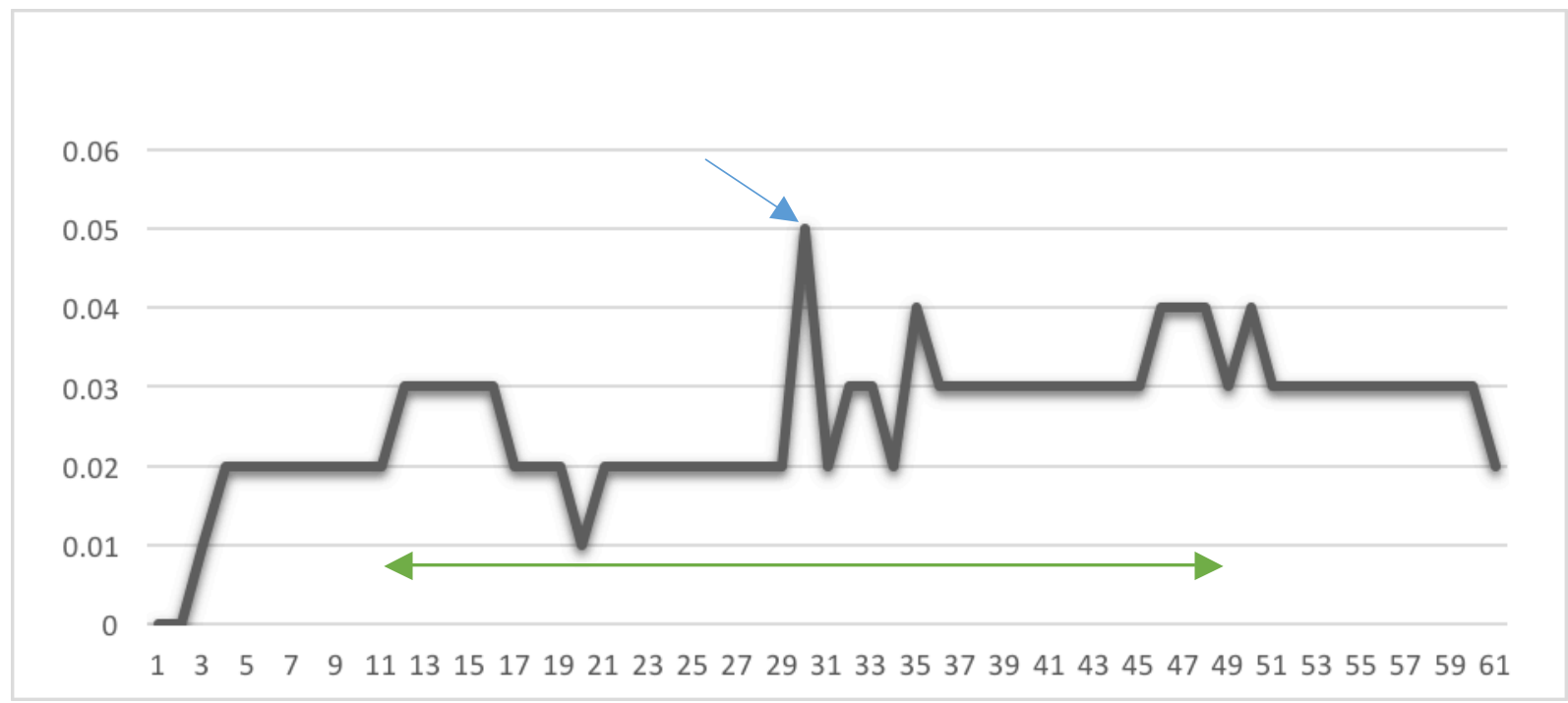

* Blue arrow: Maximum detrusor pressure * Green arrow: Total-inter-contraction interval

Fig. 15: Urodynamic recording from the study showing the different parameters measured

\subsection{Funding}

This study was funded through an internal research grant from the Department of Surgery, Western University. The protocol was approved by the Animal Use Subcommittee at Western University, London, Ontario, Canada (2012-047). All surgical procedures, follow-up, and eventual rat euthanasia were compliant with the standard operating protocols of the Animal Use Subcommittee. 


\subsection{Sample size calculation}

Since no previously- published study has used the current HA-PE carrier for BTX-A delivery, we designed this study as a pilot study. Therefore, no formal sample size calculations were done. In lieu of any formal calculations, a decision was made to have 10 rats in each experimental group. With six groups, this meant that a total of 60 rats were needed and ultimately studied.

\subsection{Data and statistical analysis}

Quantitative data analysis for the primary outcome (SNAP-25 staining) was obtained using Image $\mathrm{J}$ software after applying the same technique and method of assessment to all tissue slides. Quantitative data were expressed as means \pm standard deviations (SD) for all groups. Statistical analyses were conducted using SPSS version 23, after testing for normality, we employed one-way ANOVA, all analyses were two-tailed, and $p \leq 0.05$ considered statically significant.

Quantitative data analysis of values for the two urodynamic parameters of interest were expressed as means \pm standard deviations $(\mathrm{SD})$ for all groups. Statistical analyses were conducted using SPSS version 23. Testing of normality showed that the two urodynamic parameters values had a non-equal distribution. Therefore, non-parametric tests - the Kruskall-Wallis test for unpaired variables and Freidman test for paired variables - were employed, with all analyses two-tailed and $\mathrm{p} \leq 0.05$ considered statistically significant. 


\section{Chapter 4: Results}

All the surgical procedures undertaken were completed successfully, with no intraoperative complications. The proposed post-operative follow-up, second operative procedure and tissue harvesting were completed as planned in 59 of 60 rats. One rat, in Group \#2, was sacrificed 48 hours after the initial surgical procedure due to signs of infection and the bladder was harvested as per protocol.

In Group \#3 controls, where direct bladder muscle injection of BTX-A was performed, 8 rats exhibited visualized blebs apparent at the site of injection. The injection was deemed unsuccessful in two rats, where we suspected that the intra detrusor injection was intraluminal and therefore these 2 rats were subsequently excluded from further analysis.

Since this was a pilot study, we intended to assess our surgical technique and standardize the urodynamic study protocol. Group \#6 (BTX-A 10U-HA-PE) rats were the first group conducted. Our experience with these initial injections led to several technical modifications in the protocol. These changes and modifications are detailed below:

\subsection{Modifications to the protocol}

These modifications detailed below were designed to improve the study methodology and allow better assessment of outcomes.

\subsubsection{Modifications to the surgical technique}

Two modifications were made to the surgical technique. First, the technique to perform the urodynamic study using 2 bladder cystostomies and purse-string sutures to affix the 26-gauge angiocath and PE-50 tube led to leakage from the bladder during instillation. Therefore, we initiated urethral catheterization with a PE-50 tube and K-Y jelly applied to the external urethral meatus to avoid urethral leakage for better recording of the bladder pressure during urodynamic studies, thus avoiding the need for a second bladder incision. 
Secondly, the method of instillation was altered from using a 26-gauge angiocath placed in the bladder through a midline cystostomy incision, to performing direct bladder puncture using a $6 \mathrm{~mm} / 28$-gauge needle and gradually instilling the solution into the bladder.

\subsubsection{Modifications to the urodynamic assessment}

Performing the urodynamic assessment as initially drafted in the study protocol was technically challenging. Leakage almost always occurred at the cystostomy site, and pressure recordings were inaccurate due to reduced bladder capacity secondary to leakage.

To address these obstacles, the following changes were made. Firstly, the PE-50 tubing measuring bladder pressure was inserted urethrally rather than through a cystotomy. In addition, K-Y gel was applied to the urethral meatus to prevent urethral leakage. Secondly, a single tubing was used with two stopcocks on the pressure transducer: one connected to the PE-50 tube in the urethra and the other connected to the micro-infusion pump for infusion. The main stem of the pressure transducer has already a cord connection with the low pressure analyzer. This connection allowed for easy and straightforward recording of the urodynamic parameters of interest, preventing bladder leakage.

\subsubsection{Modifications to the injection technique}

Rat bladder is thin and it is technically hard to perform an intra- detrusor muscle injection. As such, to achieve successful injections into the detrusor muscle, a thinner calibre needle was used, replacing the $6 \mathrm{~mm} / 28$-gauge needle with a $6 \mathrm{~mm} / 30$-gauge one. This facilitated injection_into the bladder wall evidenced by well-formed blebs post injection in $8 / 10$ rats. However, this smaller needle altered how easily we could inject the viscous BTX-A-HA-PE solution. We tried different concentrations of HA-PE and found that 0.5 grams HA-PE liquefied in $2 \mathrm{ml}$ saline was the highest possible dose that could be instilled without blocking the needle. 


\subsubsection{Modifications to the BTX-A-HA-PE mixture}

There are no previous studies to assess the optimal dose of HA-PE for this study. We started by using different doses of HA-PE, beginning with 0.5 grams, which was found to be the highest possible dose of HA-PE that could be liquefied in $2 \mathrm{ml}$ saline (average rat bladder capacity). Once this was achieved, the dose of HA-PE was lowered in $0.1 \mathrm{~g}(0.4$, 0.3, 0.2 grams) to compare the effect of HA-PE dosage on BTX-A delivery.

\subsubsection{Modifications to the instillation time}

The duration of instillation was increased from 30 minutes, as initially planned in the study protocol to 60 minutes to increase the time of contact between the instilled solution and bladder mucosa.

\subsubsection{Modifications to the labeling process}

Fluorescein iso-thio-cyanate (FITC) was replaced with Alexa Fluor ${ }^{\circledR} 594$ in Group \#4 rats, because Alexa Fluor ${ }^{\circledR} 594$ molecules can attach more readily to proteins at high molar ratios without significant self-quenching, thereby enabling brighter images and increasing the sensitivity of detection (91).

\subsection{Final control and treatment group}

This experimental study involved six groups of rats, three control groups (Groups \#1-3) and three treatment groups (Groups \#4-6). Only rats in groups \#5 and \#6 had variable HA-PE doses. The Methods section (3.2) review the different group specifications. 


\section{Control groups:}

Group \#1, control arm: All rats received BTX-A 10U-saline, by instillation, which was retained in the bladder for 60 minutes (protocol modification; time of instillation modified from 30 minutes to 60 minutes).

Group \#2, control arm: All rats received HA-PE 0.5g-saline, by instillation, retained in the bladder for 60 minutes (protocol modification; time of instillation modified from 30 minutes to 60 minutes).

Group \#3, control arm: All rats received BTX-A 5U by direct intra-muscular injection. $8 / 10$ rats received a successful bladder muscle injection as evidenced by well-formed blebs.

\section{Treatment groups:}

Group \#4: All rats received Alexa Fluor®594-labeled BTX-A10U- HA-PE 0.5g, by instillation, retained in the bladder for 60 minutes (Protocol modification; Alexa Fluor ${ }^{\circledR 594}$ replaced FITC and modified time of instillation from 30 to 60 minutes). Group \#5: All rats received BTX-A 5U with HA-PE in different doses (0.2-0.5g) instilled for 60 minutes (protocol modification; time of instillation modified from 30 minutes to 60 minutes). Table 1, summarises the HA-PE doses and instillation times for Group \#5 rats. Because of the variable HA-PE doses used in Group \#5, the group was further subdivided into:

Group \#5A: rats received higher doses of HA-PE: 0.4 or 0.5 grams Group \#5B: rats received lower doses of HA-PE: 0.2 or 0.3 grams Group \#6: All rats received BTX-A 10U with different HA-PE doses (0.2-0.5g) instilled for 30 minutes (as per the protocol). Table 2, summarises the HA-PE doses and the instillation times for Group \#6. Because of the variable HA-PE doses used in Group \#6, this group was further sub-divided into:

Group \#6A: rats received higher doses of HA-PE: 0.4 or 0.5 grams Group \#6B: rats received lower doses of HA-PE: 0.2 or 0.3 gram 


\begin{tabular}{|ll|l|l|}
\hline Rat number & Group & \multicolumn{1}{|c|}{ HA-PE dose } & Instillation time \\
\hline 1st rat & $(5 \mathrm{~A})$ & $0.4 \mathrm{~g}$ & 60 minutes \\
\hline 2nd rat & $(5 \mathrm{~A})$ & $0.4 \mathrm{~g}$ & 60 minutes \\
\hline 3rd rat & $(5 \mathrm{~A})$ & $0.4 \mathrm{~g}$ & 60 minutes \\
\hline 4th rat & $(5 \mathrm{~A})$ & $0.5 \mathrm{~g}$ & 60 minutes \\
\hline 5th rat & $(5 \mathrm{~B})$ & $0.3 \mathrm{~g}$ & 60 minutes \\
\hline 6th rat & $(5 \mathrm{~B})$ & $0.2 \mathrm{~g}$ & 60 minutes \\
\hline 7th rat & $(5 \mathrm{~B})$ & $0.2 \mathrm{~g}$ & 60 minutes \\
\hline 8th rat & $(5 \mathrm{~B})$ & $0.2 \mathrm{~g}$ & 60 minutes \\
\hline 9th rat & $(5 \mathrm{~B})$ & $0.3 \mathrm{~g}$ & 60 minutes \\
\hline 10th rat & $(5 \mathrm{~B})$ & $0.2 \mathrm{~g}$ & 60 minutes \\
\hline
\end{tabular}

Table 1: Doses and instillation times in group 5 rats 


\begin{tabular}{|ll|l|l|}
\hline Rat number & Group & \multicolumn{1}{|c|}{ HA-PE dose } & Instillation time \\
\hline $1^{\text {st }}$ rat & $(6 \mathrm{~A})$ & $0.4 \mathrm{~g}$ & 30 minutes \\
\hline $2^{\text {nd }}$ rat & $(6 \mathrm{~A})$ & $0.5 \mathrm{~g}$ & 30 minutes \\
\hline $3^{\text {rd }}$ rat & $(6 \mathrm{~A})$ & $0.5 \mathrm{~g}$ & 30 minutes \\
\hline $4^{\text {th }}$ rat & $(6 \mathrm{~A})$ & $0.5 \mathrm{~g}$ & 30 minutes \\
\hline $5^{\text {th }}$ rat & $(6 \mathrm{~A})$ & $0.4 \mathrm{~g}$ & 30 minutes \\
\hline $6^{\text {th }}$ rat & $(6 \mathrm{~B})$ & $0.3 \mathrm{~g}$ & 30 minutes \\
\hline $7^{\text {th }}$ rat & $(6 \mathrm{~B})$ & $0.3 \mathrm{~g}$ & 30 minutes \\
\hline $8^{\text {th }}$ rat & $(6 \mathrm{~B})$ & $0.3 \mathrm{~g}$ & 30 minutes \\
\hline $9^{\text {th }}$ rat & $(6 \mathrm{~B})$ & $0.2 \mathrm{~g}$ & 30 minutes \\
\hline $10^{\text {th }}$ rat & $(6 \mathrm{~B})$ & $0.3 \mathrm{~g}$ & 30 minutes \\
\hline
\end{tabular}

Table 2: Doses and instillation times in group 6 rats

\subsection{Exclusions from analysis and explanation}

\subsubsection{Exclusions from SNAP-25 staining analysis}

SNAP-25 staining were evaluated in all rat groups, with the exception of Group 4 (10 rats) in which Alexa Fluor ${ }^{8} 594-$ labelling was assessed. Another two rats were excluded from Group 3 due to unsuccessful BTX-A direct detrusor injections, a total of 48 rats out of 60 were assessed for SNAP-25 staining percentage. 


\subsubsection{Exclusions from urodynamic analysis}

Group 6 rats were excluded from urodynamic analysis, as we were unable to perform adequate bladder filling due to significant bladder leakage as a result of the 2 cystostomies technique into the bladder. This initial experience led to urodynamic protocol modifications as detailed above (4.1). Two rats were also excluded from Group 3 due to unsuccessful BTX-A detrusor injections and one rat from group 2 was sacrificed after 48 hours due to infection. Consequently, the urodynamic assessment ultimately was performed in 47 of the 60 rats.

\subsection{Primary outcome analysis SNAP-25 staining}

Five groups were evaluated for SNAP-25 staining; control groups 1- 3 and treatment groups 5 and 6. SNAP-25 staining was identified in both arms of the study with variable density. We used SNAP-25 staining in the tissue slides as a primary outcome measure of BTX-A action because SNAP-25 cleavage is the primary action of BTX-A in order to end up with muscle relaxation.

Group 1 (BTX-A 10U- Saline) and Group 2 (HA-PE 0.5g - saline) instillations yielded the highest density of SNAP-25 staining, with mean staining percentages of $21.8 \pm 6.5 \%$ and $24.6 \pm 12.3 \%$, respectively, which was confirmed in both bladder urothelium and detrusor muscle, thereby indicating the lack of any substantial SNAP-25 cleavage and therefore as expected, absence of any appreciable BTX-A activity. Conversely, Group 3 control rats (BTX-A 5U IM injection) exhibited the lowest density of SNAP-25 staining, with a mean staining percentage of $(7.3 \pm 5.0 \%)$ which suggests substantial SNAP-25 cleavage and considerable BTX-A activity. This extensive SNAP-25 cleavage confirms that direct BTX-A injection into the bladder muscle remains the gold standard method to deliver BTX-A to the detrusor muscle.

Group 5 rats (BTX-A 5U- HA-PE 0.2-0.5g, 60-minute instillation) exhibited SNAP25 cleavage dependent on the dose of HA-PE. Group 5A rats $(n=4)$ which had received a higher dose of HA-PE (0.4 or 0.5g), exhibited minimal SNAP-25 staining, with a mean 
staining percentage of $12.4 \pm 12.3 \%$, which was noticeably lower than in group 5B rats $(n=6)$ that received lower $(0.2,0.3 \mathrm{~g})$ HA-PE dose, in which the mean SNAP-25 staining percentage was $23.9 \pm 8.5 \%$.

Group 6 rats, in which BTX-A 10U-HA-PE was instilled for 30 minutes, displayed minimal BTX-A activity, indicated by a relatively high degree of SNAP-25 staining in all tissue slides and showed HA-PE dose dependent response as well. Group 6A rats $(n=5)$ exhibited a mean SNAP-25 staining percentage of $22.9 \pm 10.2 \%$, versus $31.5 \pm 3.5$ in Group 6B $(n=5)$.

Table 3, lists the mean SNAP-25 staining percentages in the different groups, along with standard deviations.

Figure 16 and 17, illustrate SNAP-25 staining of all groups compared to group 3 controls.

Figure18, illustrates SNAP-25 staining of groups 5A\&5B and groups 6A\&6B compared to group 3 controls. 


\begin{tabular}{|c|c|}
\hline Group & Mean Staining Percentage \pm SD \\
\hline Group 1 (BTX-A 5 IU-saline) & $21.8 \pm 6.5$ \\
\hline \multicolumn{2}{|l|}{60 minutes } \\
\hline Group 2 (HA-PE, $0.5 \mathrm{~g}$ ) & $24.6 \pm 12.3$ \\
\hline \multicolumn{2}{|l|}{60 minutes } \\
\hline Group 3 (BTX-A 5 U IM injection) & $7.3 \pm 5.0$ \\
\hline Group 5 A (BTX-A 5 U-HA-PE, 0.4,0.5g) & $12.4 \pm 12.3$ \\
\hline 60 minutes & \\
\hline Group 5 B (BTX-A 5U-HA-PE, 0.2,0.3g) & $23.9 \pm 8.5$ \\
\hline 60 minutes & \\
\hline Group 6A (BTX-A 10 U-HA-PE, $0.4,0.5 \mathrm{~g}$ ) & $22.9 \pm 10.2$ \\
\hline 30 minutes & \\
\hline Group 6B (BTX-A 10 U-HA-PE, 0.2,0.3g) & \\
\hline 30 minutes & $31.5 \pm 3.5$ \\
\hline
\end{tabular}

Table 3: Mean SNAP-25 staining percentages \pm SD of in all groups 


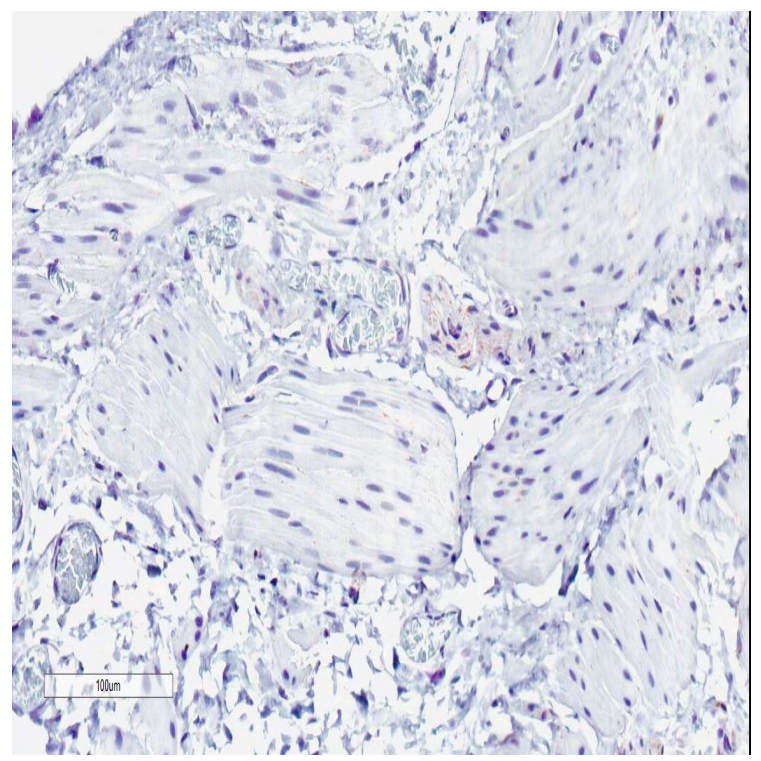

Group 3

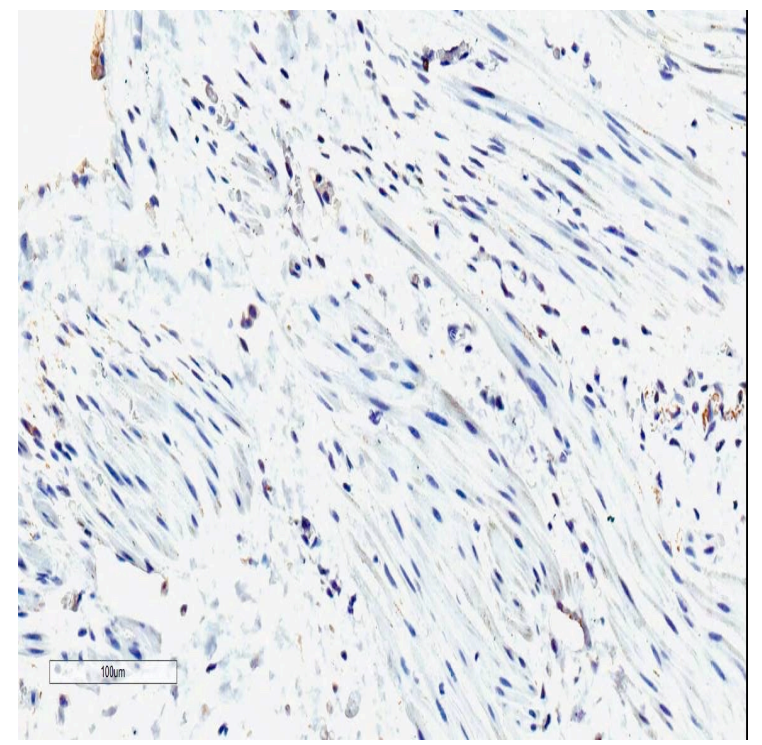

Group 3

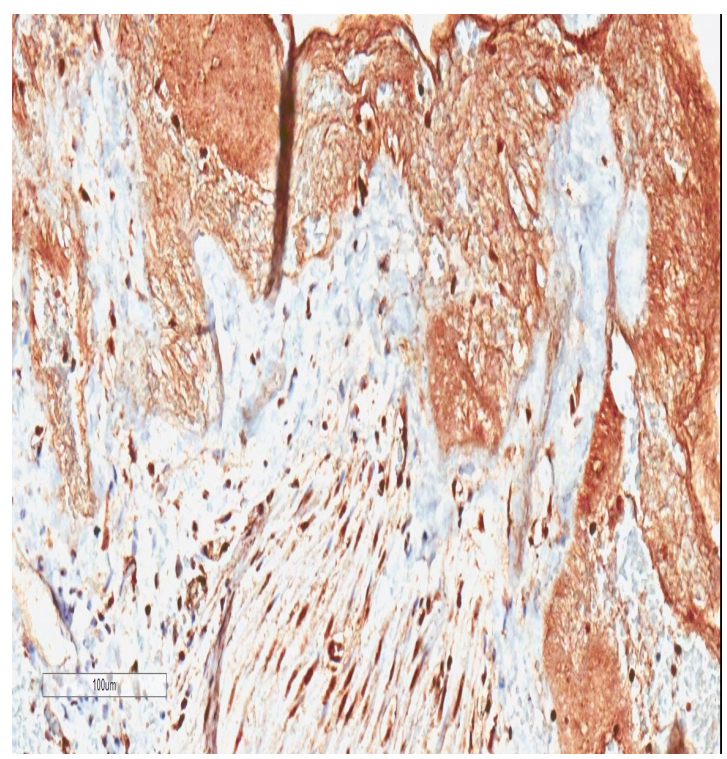

Group 1

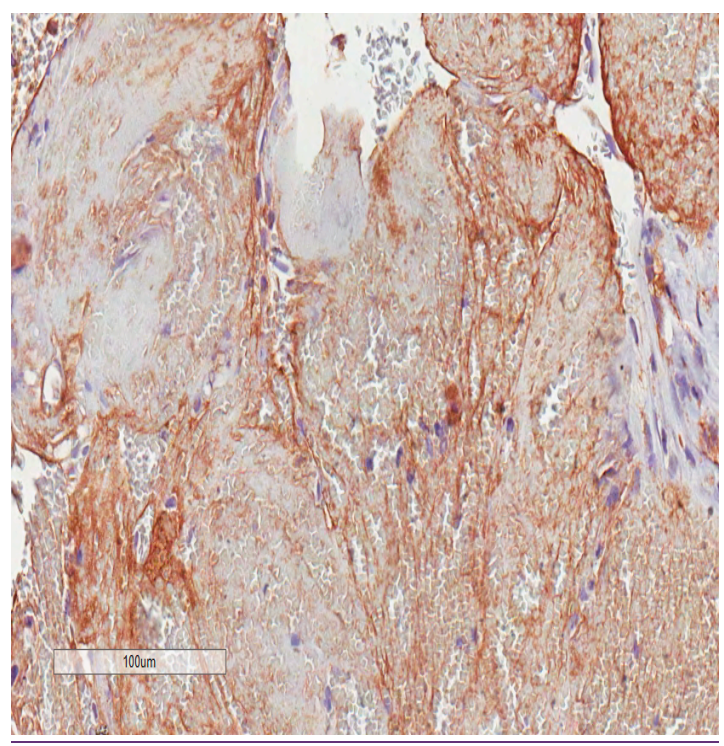




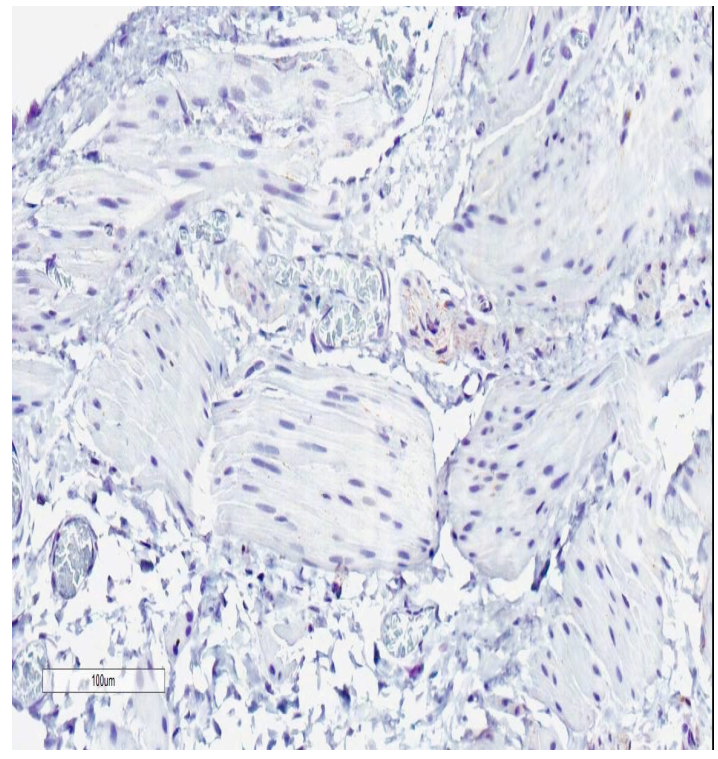

Group 3

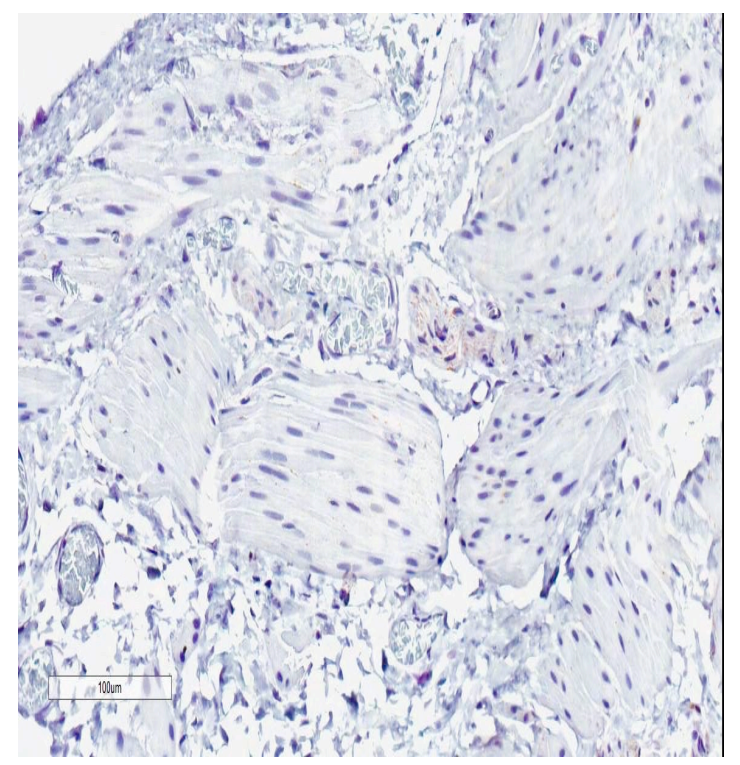

Group 3

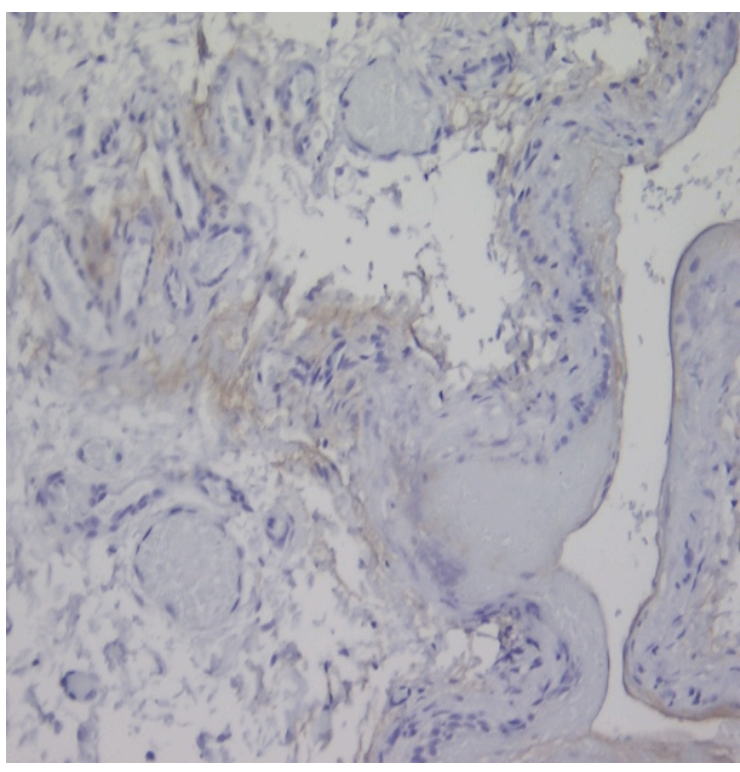

Group 5A

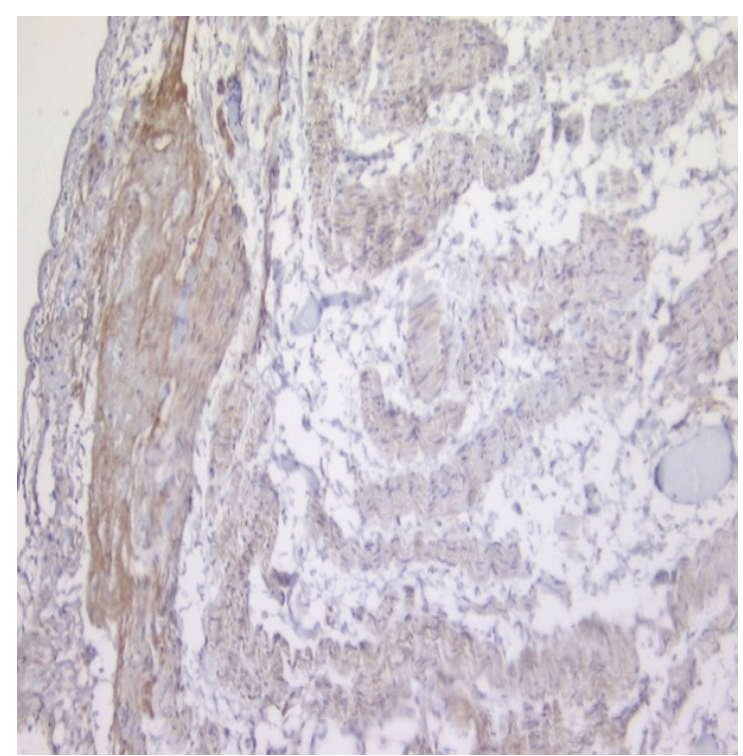




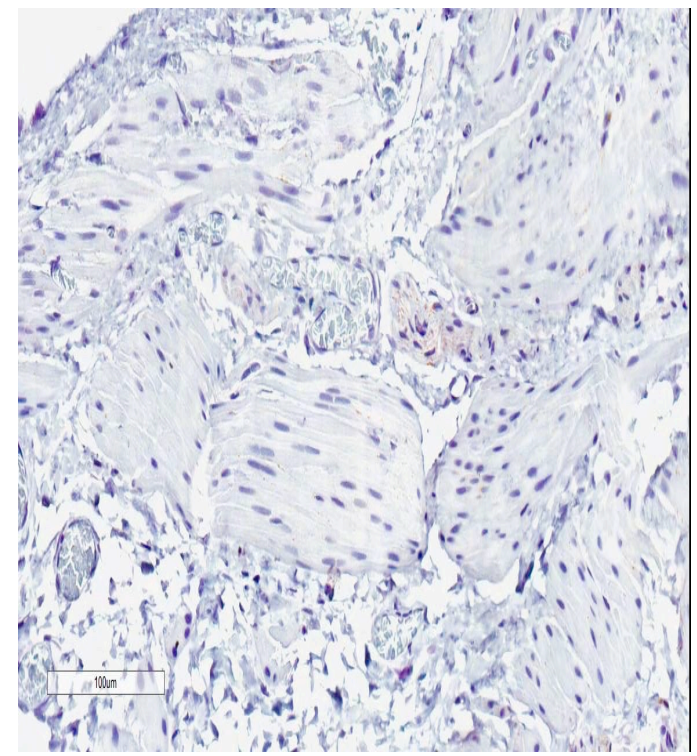

Group 3

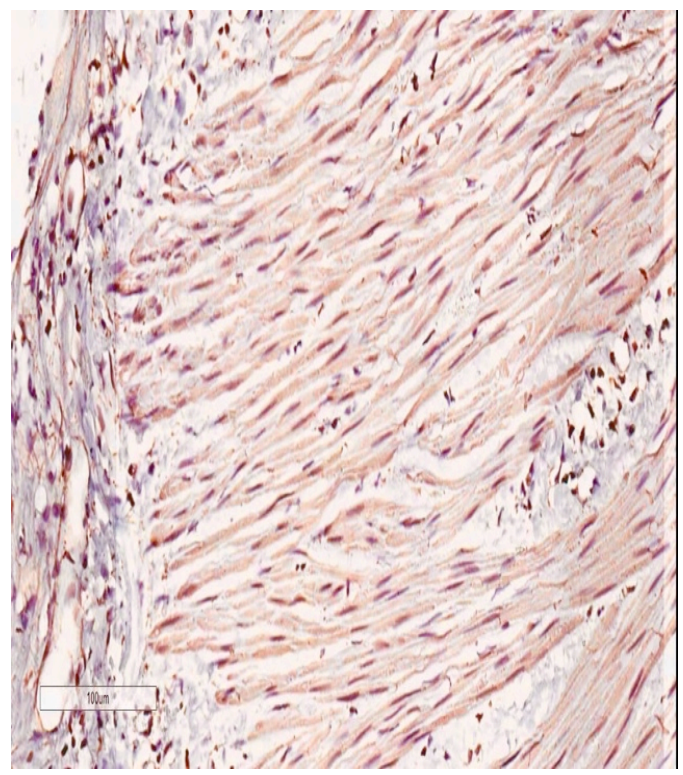

Group 6A

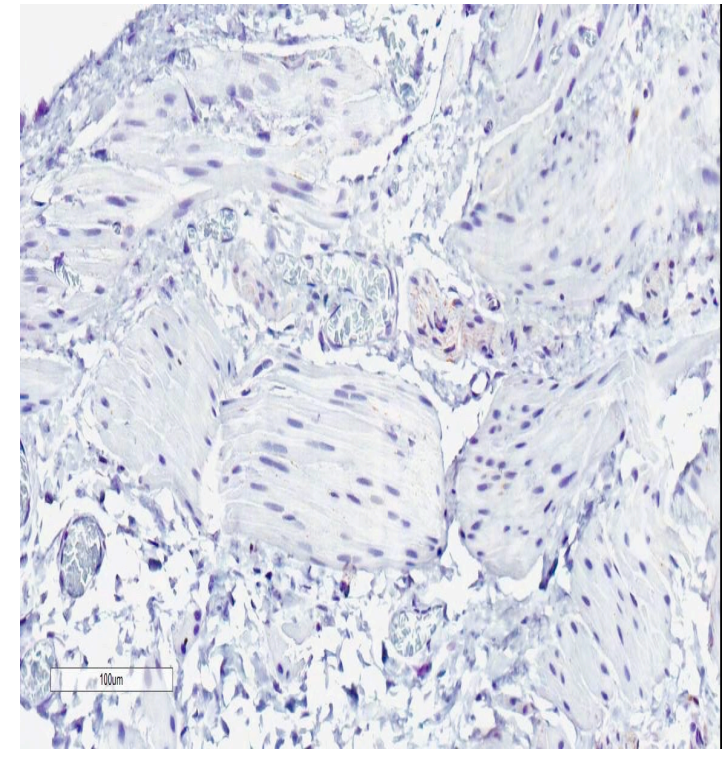

Group 3

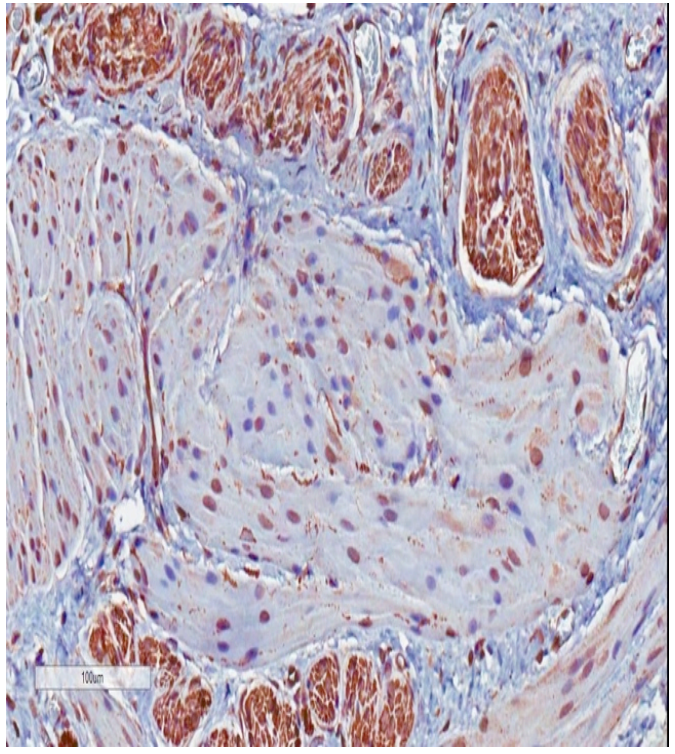

Group 6B

Fig 16: SNAP-25 staining in all treatment and control groups compared

to group 3 controls, magnification $20 \times$ 


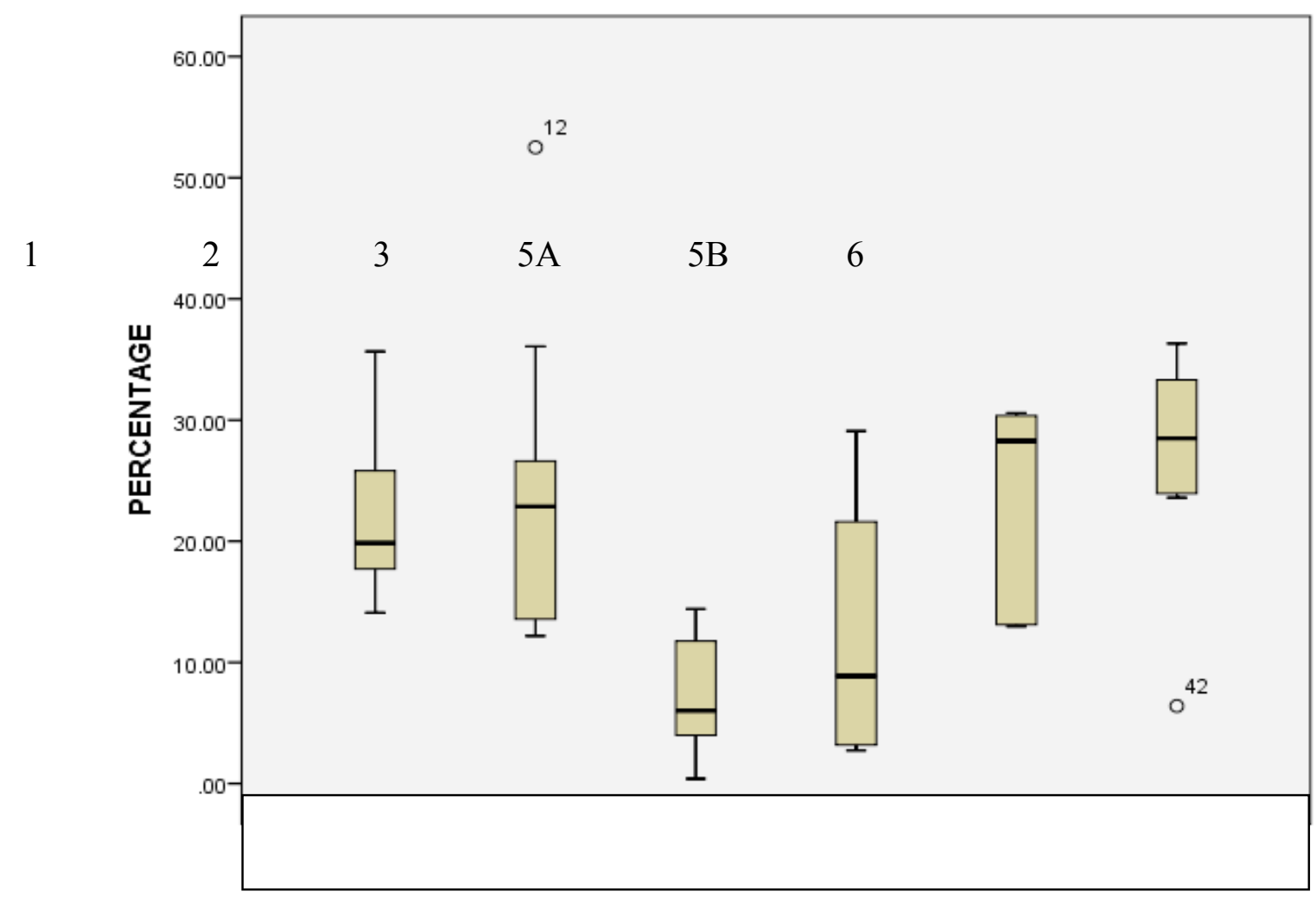

Fig. 17: Mean percentage of SNAP-25 Staining. Groups 1- 6 


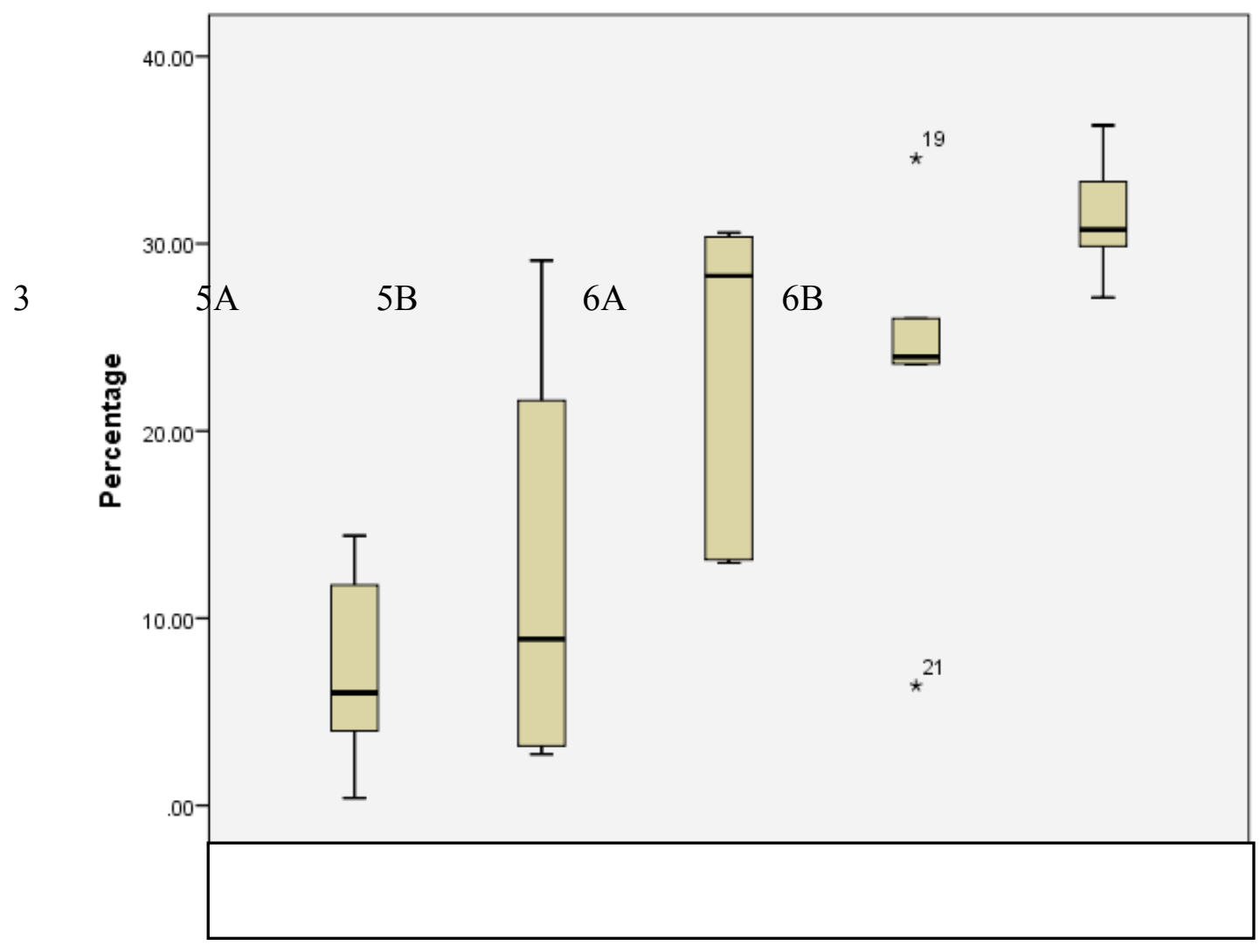

Fig. 18: Mean percentage of SNAP-25 Staining. Groups 3,5 and 6 


\begin{tabular}{|c|c|c|c|}
\hline Group & Group & Std. Error & Significance \\
\hline 3 & $\begin{array}{c}1 \\
\text { (BTX-A } 10 \mathrm{U}- \\
\text { saline) }\end{array}$ & 4.3 & 0.02 \\
\hline 3 & $\begin{array}{c}2 \\
\text { (HA-PE } 0.5 \mathrm{~g} \text { saline) }\end{array}$ & 4.3 & 0.003 \\
\hline 3 & $\begin{array}{c}5 \mathrm{~A} \\
\text { BTX-A5U-HA-PE } \\
(0.4,0.5 \mathrm{~g})\end{array}$ & 4.8 & 1.000 \\
\hline 3 & $\begin{array}{c}5 \mathrm{~B} \\
\text { BTX-A 5U-HA-PE } \\
(0.2,0.3 \mathrm{~g})\end{array}$ & 4.3 & 0.008 \\
\hline 3 & $\begin{array}{c}6 \mathrm{~A} \\
\text { BTX-A10 U-HA-PE } \\
(0.4,0.5 \mathrm{~g})\end{array}$ & 4.5 & 0.02 \\
\hline 3 & $\begin{array}{c}6 \mathrm{~B} \\
\text { BTX-A10 U-HA-PE } \\
(0.4,0.5 \mathrm{~g})\end{array}$ & 4.5 & $<0.001$ \\
\hline
\end{tabular}

*. The mean difference is significant at a $\mathrm{p}<0.05$ level.

Table 4: SNAP-25 staining percentage comparisons using one-way analysis of variance (ANOVA) compared to BTX-A 5U detrusor injection (Group 3) 
There was evidence of a statistically-significant increase in SNAP 25 cleavage comparing Group 3 rats to either Groups 1 or 2 ( $p=0.02$ and 0.003 , respectively), or to group 5B with $(p=0.008)$.

On the other hand, comparing the mean percentage of SNAP-25 staining between Group 3 and group $5 \mathrm{~A}$, failed to identify any statistically-significant difference $(\mathrm{p}=1.00)$ suggesting that the mean SNAP-25 staining in these two groups was comparable and evidence of similar BTX activity in Group 5A compared to Group 3.

Similarly, comparing Group 3 mean SNAP-25 staining percentage to group 6A (BTX-A $10 \mathrm{U}$ instilled for 30 minutes with a high HA-PE dose (0.4 and 0.5) and to group 6B (similar BTX dose and instillation time but lower HA-PE dose 0.2 and $0.3 \mathrm{~g}$ ) revealed statistically significant difference in mean SNAP-25 staining in Group 6A and 6B versus Group 3 ( $p=0.02, p<0.001$, respectively), showing that despite higher dose of BTX, the reduced instillation time of 30 minutes may be a significant factor to hamper BTX translocation. Collectively, these results show that both prolonged instillation time and higher dose HA-PE dose should be combined together to facilitate BTX-A delivery across the urothelium with this novel delivery mechanism. Higher dose of BTX-A might has an additional effect but this point was not possibly obviated by the instillation time.

\subsection{Secondary outcome analyses}

\subsubsection{Alexa ${ }^{\circledR} 594$ fluorescence}

In Group 4 rats, where BTX-A10U labelled with Alexa Fluor®594 and HA-PE $0.5 \mathrm{~g}$ was instilled for 60 minutes, positive red florescence was observed in the urothelium upon examination under a confocal microscope. These slides were compared by a blinded examiner to similarly-processed frozen section specimens from Group 1 rats that had undergone BTX-A-saline instillation for 60 minutes without Alexa Fluor ${ }^{\circledR 594 . ~ G r o u p ~} 1$ exhibited the absence of Alexa Fluor ${ }^{\circledR 594}$ red florescence that was clearly detected on Group 4 tissue slides. These results indicate that BTX-A $10 \mathrm{U}$ instilled with HA-PE $0.5 \mathrm{~g}$ for 60 minutes was successfully translocated a cross the urothelium denoted by positive Alexa ${ }^{\circledR 594}$ red fluorescence in group 4 tissue slides, compared to controls, (Figure19). 


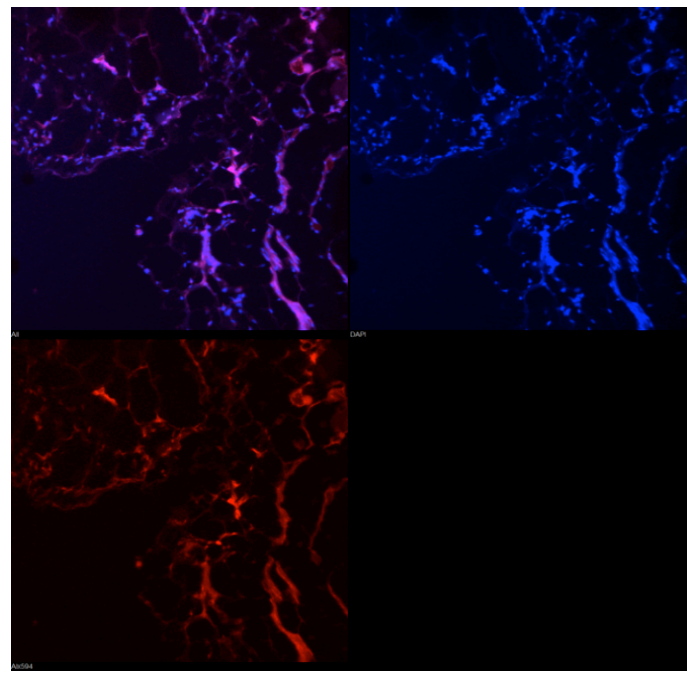

A

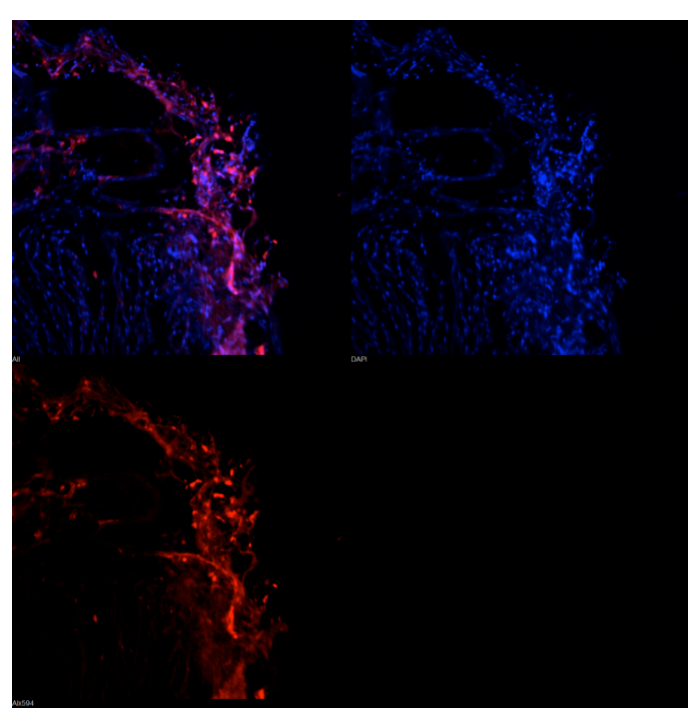

$\mathrm{C}$

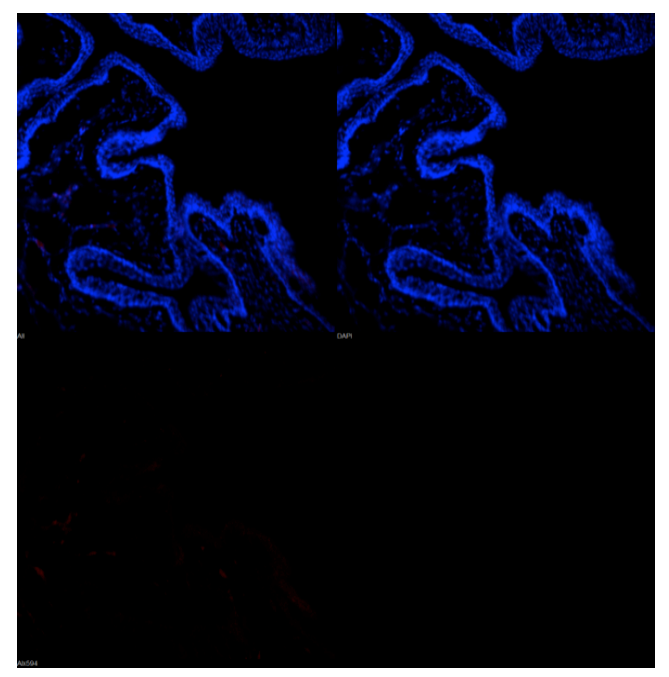

B

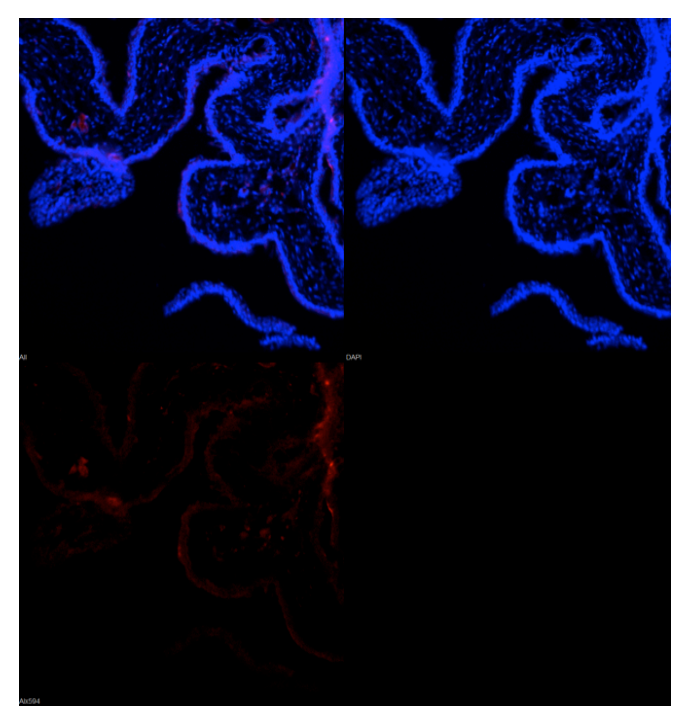

$\mathrm{D}$

Fig. 19: Group 4: Alexa Fluor®594-labeled BTX-A-HA-PE (A \& C)

Group 1(control): BTX-A-saline (B \& D). 


\subsubsection{Urodynamic results}

Urodynamic evaluation was performed in all control groups and experimental groups, at the initial procedure without acetic acid (AA) instillation and after two weeks, following instillation of $1 \%$ acetic acid for 30 minutes. The urodynamic studies in Group 6 were excluded because of technical errors resulting in leakage of the fluid. All urodynamic studies were performed under anesthesia through a urethral PE-50 tube connected to the urodynamic machine.

\subsubsection{Maximum Pressure}

\begin{tabular}{|c|c|c|}
\hline Group & Maximum Pressure & $\begin{array}{c}\text { Maximum Pressure } \\
\text { (Follow up study after AA } \\
\text { instillation) }\end{array}$ \\
\hline $\begin{array}{c}\text { Group 1 } \\
\text { (n=10) }\end{array}$ & $0.02 \pm 0.01$ & $0.04 \pm 0.02$ \\
\hline $\begin{array}{c}\text { Group 2 } \\
\text { (n=9) }\end{array}$ & $0.03 \pm 0.008$ & $0.04 \pm 0.02$ \\
\hline $\begin{array}{c}\text { Group 3 } \\
\text { (n=8) }\end{array}$ & $0.02 \pm 0.004$ & $0.04 \pm 0.009$ \\
\hline $\begin{array}{c}\text { Group 4 } \\
\text { (n=10) }\end{array}$ & $0.03 \pm 0.01$ & $0.04 \pm 0.01$ \\
\hline $\begin{array}{c}\text { Group 5A } \\
\text { (n=4) }\end{array}$ & $0.02 \pm 0.005$ & $0.05 \pm 0.009$ \\
\hline $\begin{array}{c}\text { Group 5B } \\
\text { (n=6) }\end{array}$ & & 0.008 \\
\hline
\end{tabular}

Table 5: Mean maximum pressure at baseline and after AA instillation, \pm SD 
We did not observe any significant differences in baseline MP at the time of initial urodynamic study in the control versus treatment groups $(p=1.0)$, table (5). The administration of acetic acid induced an increase in the MP in all groups. However, there again was no significant difference in maximum pressure after acetic acid instillation between the groups $(p=0.2)$. Kruskal-Wallis test was used to evaluate the significance of MP in both baseline and after acetic acid instillation, (Fig.20, 21).

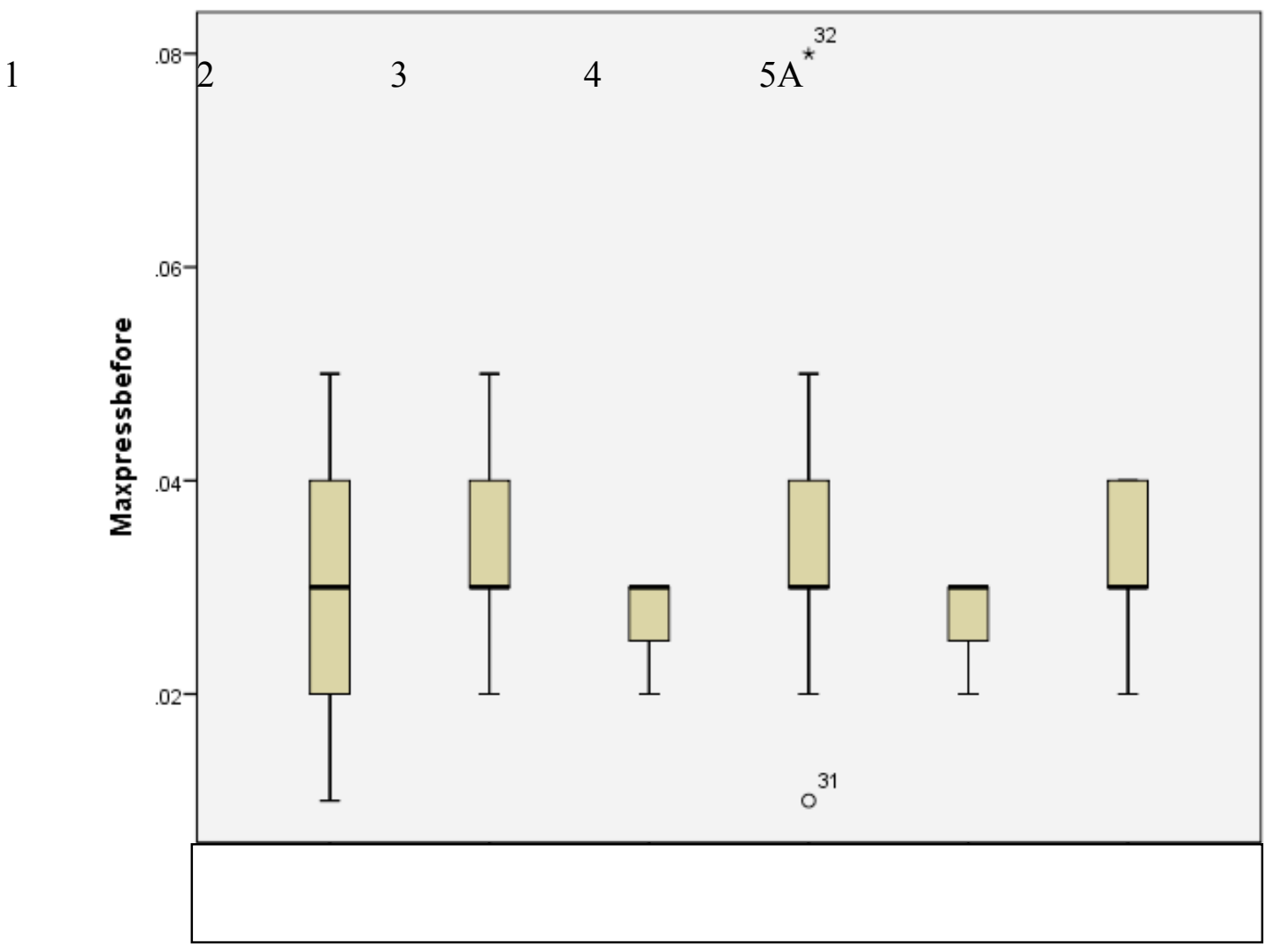

Fig. 20: Maximum pressure at baseline urodynamic study 


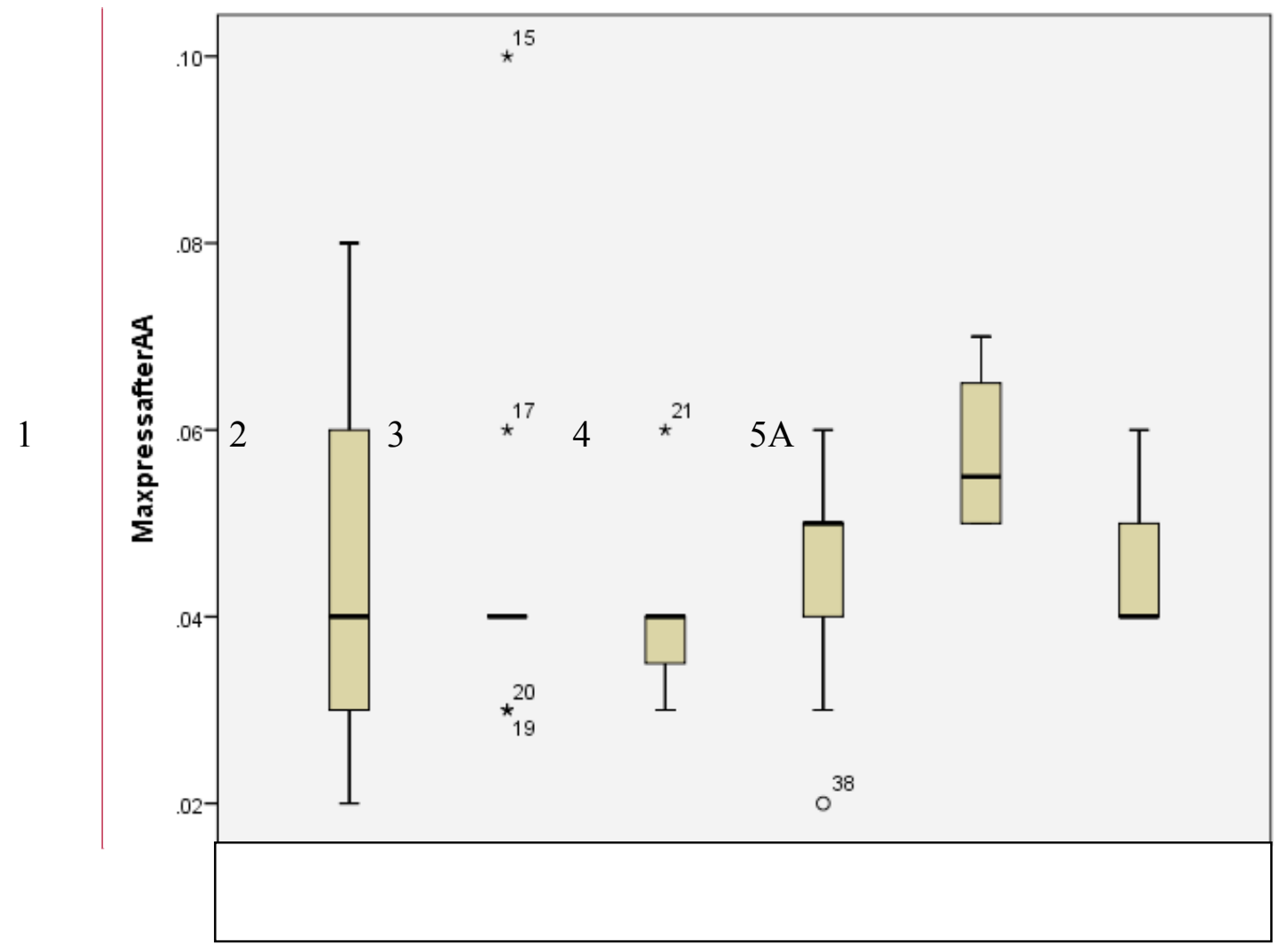

Fig. 21: Maximum pressure after 1\% acetic acid instillation on follow up urodynamic study 


\subsubsection{Inter-contraction interval (ICI)}

No significant differences were noted in baseline inter-contraction intervals between the six groups $(\mathrm{p}=0.9)$. As expected, control groups (1 and 2) showed shortening of intercontraction intervals at the time of the second urodynamic assessment, after $1 \%$ acetic acid instillation for 30 minutes. Group 3 rats showed prolongation of inter-contraction intervals compared to the baseline despite AA stimulation, demonstrating the physiological effects of BTX-A $(13.0 \pm 11.4$ seconds at baseline $\& 16.8 \pm 10.2$ seconds after AA).

Group 5A was the only treatment group that exhibited a relatively stable inter-contraction intervals, during the second urodynamic assessment after AA instillation mimicking the results we showed with group $3(11.8 \pm 4.3$ at baseline $\& 11.3 \pm 7.0$ after AA). This effect did not reach statistical significance. Statistical analysis using the non-parametric Kruskal-Wallis test identified significant differences in ICI at day \#14 between the groups $(\mathrm{p}=0.01)$, (Table 6, Fig. 22,23). 


\begin{tabular}{|l|c|c|}
\hline Group & ICI at baseline & ICI after AA instillation \\
\hline Group 1 & $9.6 \pm 2.1$ & $5.1 \pm 3.7$ \\
\hline Group 2 & $10.7 \pm 4.7$ & $8.9 \pm 3.2$ \\
\hline Group 3 & $13.0 \pm 11.4$ & $16.8 \pm 10.2$ \\
\hline Group 4 & $16.0 \pm 11.6$ & $11.3 \pm 5.1$ \\
\hline Group 5A & $11.8 \pm 4.3$ & $11.3 \pm 7.0$ \\
\hline Group 5B & $15.2 \pm 9.8$ & $6.3 \pm 2.6$ \\
\hline
\end{tabular}

Table 6: Mean inter-contraction intervals (ICI) at baseline and at 14 days after AA instillation; $\pm \mathrm{SD}$ 


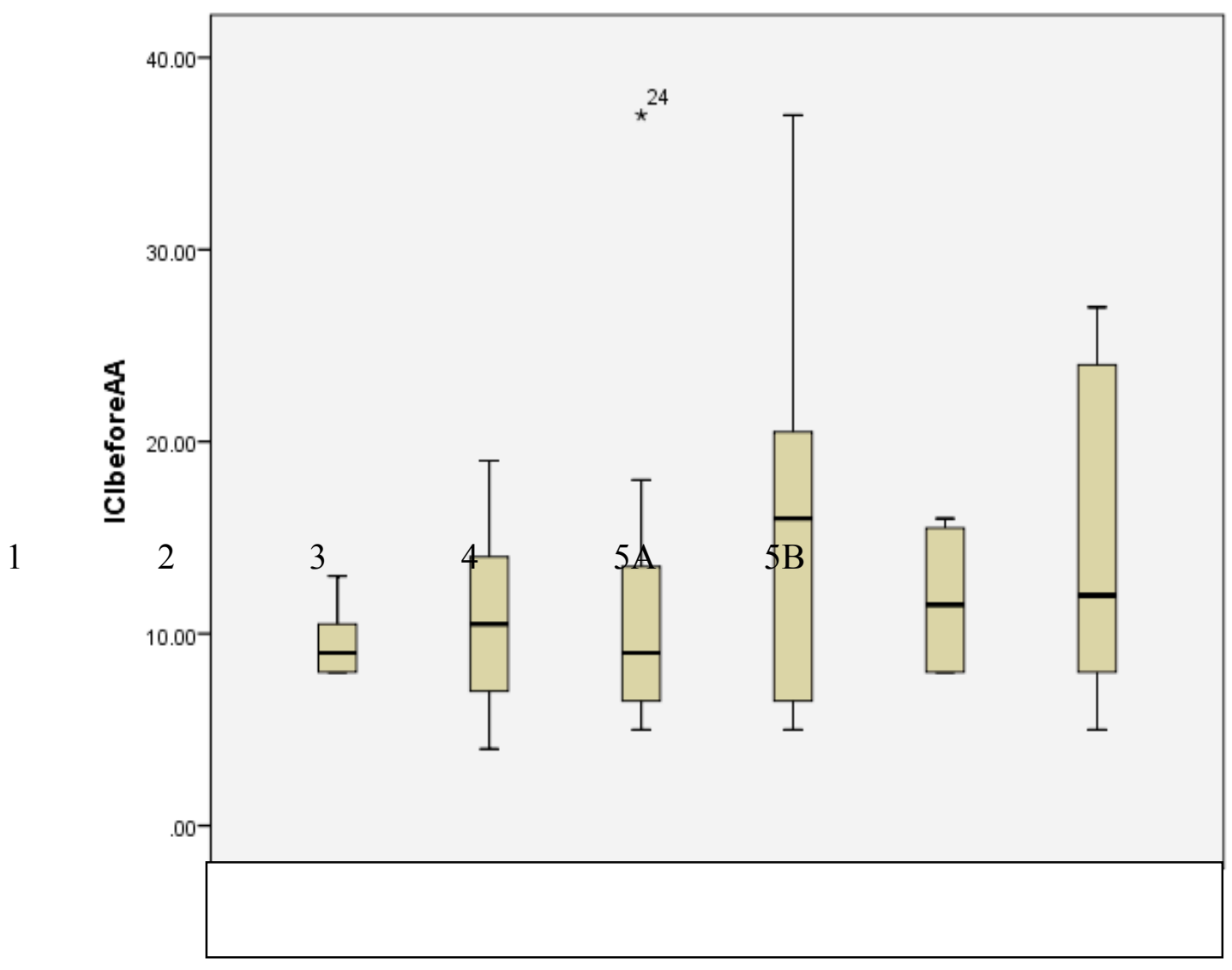

Fig. 22: Baseline ICI urodynamic study 


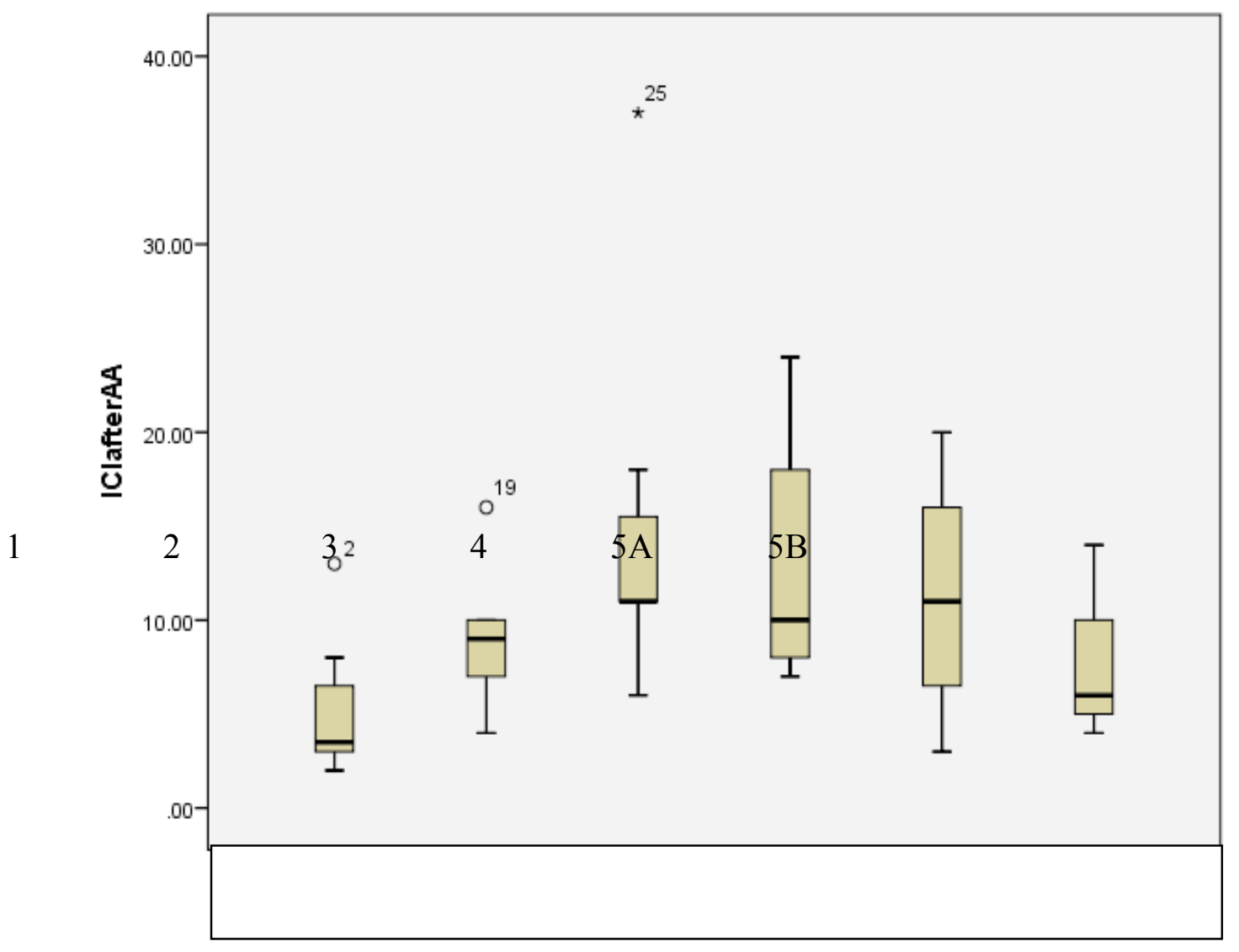

Fig. 23: ICI after acetic acid instillation on follow up urodynamic study at day 14

Group \# 4 rats, showed considerable shortening of ICI after 1\% acetic acid instillation at day 14 compared to baseline $(16.0 \pm 11.6$ at baseline $\& 11.3 \pm 5.1$ after AA) despite that we used BTX-A $10 \mathrm{U}$ for 60 minutes instillation. We do not have adequate explanation of this point; the labeling process might deem responsible for this effect and it is possible 
that the labeling process might by blocking the active functional sites of BTX-A molecule.

Interestingly, in agreement with our SNAP-25 cleavage results, group 5B rats showed considerable shortening of ICI after $1 \%$ acetic acid instillation compared to baseline $(15.2$ \pm 9.8 at baseline $\& 6.3 \pm 2.6$ after AA) suggesting the lack of BTX-A action most probably due to low HA-PE dosage and a possible increased bladder reactivity to acetic acid instillation.

In conclusion; SNAP-25 staining results of our experimental study show that bladder instillations of BTX-A with HA-PE carrier led to significant histological evidence of SNAP-5 cleavage and BTX-A translocation a cross the urothelium comparable to direct muscle injection (Group 3).

We demonstrated another evidence of BTX-A translocation in group \#4 rats using BTXA labeled with Alexa Fluor ${ }^{\circledR 594}$. HA-PE dose and instillation time had an impact on BTX-A translocation and effect. Clear evidence of BTX-A action on urodynamic data is not clear and The study showed weak evidence of the relaxing effect of BTX-A-HA-PE instillation on detrusor muscle, yet our data shows encouraging results that could be more evident in future studies on bigger animals with bigger sample sizes. 


\section{Chapter 5: Discussion}

The current study, in a rat model, assessed the success of the carrier, hyaluronan phosphatidyl-ethanolamine (HA-PE), in the delivery of botulinum toxin A (BTX-A) to bladder muscle when administered by bladder instillation rather than by the standard clinical practice of direct bladder muscle injection. The underlying hypothesis was that HA-PE might facilitate the passage of BTX-A across the normally impermeable urothelium that forms the innermost lining of the bladder wall.

\section{Summary of study findings and comparative literature}

As expected, we found that simple instillations of BTX-A in saline were ineffective at delivering BTX-A across the urothelium. Using SNAP-25 staining as a marker of effective BTX-A passage through the urothelium, we histologically observed that simple BTX-A - saline solution failed to result in the cleavage of SNAP-25 in a control group of rats (group1), as evidenced by the high mean percentage of SNAP-25 staining relative to rats in which direct detrusor injection of BTX-A was done (group 3). These results confirm those published by Chuang et al who assessed the delivery of BTX-A in rat bladders by instillation and identified both histological and urodynamic evidence that BTX-A in saline alone was ineffective (80).

Group 3 rats which received direct bladder muscle injection exhibited the least mean staining percentage of SNAP-25 of all the six groups, indicating the greatest degree of BTX-A- induced SNAP-5 cleavage. This group also was the only one to experience prolongation of the inter-contraction intervals on follow up urodynamic studies, despite acetic acid stimulation, suggesting decreased detrusor muscle hyperactivity. Several other investigators had already studied the effectiveness of intra-detrusor injections of BTX-A in humans and the results have largely been supportive $(70,71)$. 
Consequently, based upon those of previously-published research, for our purposes; the control group that received direct detrusor injections was the reference group against which all others were compared.

We detected no evidence of inflammation in the form of inflammatory cells and/or disruption of cell integrity, in the urothelium or detrusor muscle after the administration of HA-PE with saline (group 2), obviating any concerns that this preparation may lead to bladder inflammation as a potential factor in allowing the BTX-A translocation rather than a direct effect of HA-PE. This is conceivable because the HA-PE carrier is formed from two biological components - hyaluronic acid and phospholipids - both of which are integral components of body tissues. As such, it should unlikely be toxic to the bladder epithelium or to trigger a foreign body immune response.

Treatment group \#5 was further subdivided into rats that received a higher dose of HA-PE ( 0.4 or $0.5 \mathrm{~g})$, and those that received a lower dose of HA-PE $(0.2$ or $0.3 \mathrm{~g})$. We found that the mean percentage of SNAP-25 staining was comparable in the sub-group of rats that received 5U BTX-A plus higher-dose HA-PE (either 0.4 or $0.5 \mathrm{~g}$ ) instilled for 60 minutes, to those rats that received the direct detrusor injections, as indicated by mean SNAP-25 staining percentages of $12.4 \%$ versus $7.3 \%$, respectively $(\mathrm{p}=1.00)$. Those rats in which BTX-A was instilled accompanied by a lower dose of HA-PE (between 0.2 and $0.3 \mathrm{~g}$ ) fared no better than those administered BTX-A in saline alone, these mean percentages of SNAP staining being 23.9 and $21.8 \%$, respectively. Therefore, the dose of HA-PE played a fundamental role in the effective delivery of BTX-A.

These results provide promising evidence that BTX-A instillation, when combined with a high dose of HA-PE (in the range of 0.4 to $0.5 \mathrm{~g}$ in $2 \mathrm{ml}$ saline) and instillation time of at least (60 minutes), could be a promising alternative therapy in the future to BTX-A injection in neurogenic bladder dysfunction, if future studies confirm our current results.

By facilitating the transfer of BTX-A through urothelium, our newly-formulated carrier has the potential to augment the efficacy of intravesical BTX-A instillation to reach similar therapeutic effect as direct muscle injection. This conjecture, obviously, requires 
further investigation and reproduction to assess the effect in larger animals with thicker bladder walls and an understanding of the HA-PE dose effect and ideal instillation time.

In treatment group\# 4 we identified additional evidence that BTX-A was able to cross the urothelium when combined with a higher-dose HA-PE using fluorescence evidence. This was the detection of Alexa Fluor ${ }^{\circledR 594-l a b e l e d ~ B T X-A ~ i n ~ r a t ~ b l a d d e r ~ w a l l ~ t i s s u e ~ a f t e r ~} 60$ minutes of instillation of BTX-A 10 U- HA-PE $0.5 \mathrm{~g}$. Such fluorescence was absent in control rats in which BTX-A 10U-saline was instilled and maintained in the bladder for 60 minutes before draining. The evidence here is tainted by the fact that good tissue architecture was not obtained during our frozen section examinations. Therefore, while we are confident of the fluorescence observed in the bladder epithelium, it was hard to confirm the exact depth of tissue penetration into the bladder muscle. Nevertheless, the presence of fluorescence in the deeper layers of the epithelium is preliminary proof of our principle and hypothesis.

One of our initial goals was also to see if BTX-A delivery using the newly-formulated HA-PE carrier resulted in any physiological improvements in bladder wall function after noxious stimuli using acetic acid. We sought to determine whether BTX-A-HA-PE instillation would reduce the extent of bladder hyperactivity measured by ICI induced by instilling $1 \%$ acetic acid for 30 minutes during our follow up urodynamic procedure. We expected that successful delivery of BTX-A would prolong the inter-contraction interval (ICI) despite acetic acid stimulation. The only rats that experienced mean prolongation in the ICI was the group treated by BTX-A intra-detrusor injection (group 3), among which the ICI rose from 13.0 to 16.8 milliseconds. Understandably, acetic acid triggered a marked shortening of the ICI in all the remaining installation groups, with the exception of those given BTX-A-HA-PE at a higher dose and longer instillation time (5A), in which the ICI essentially remained unchanged. This again suggests that higher HA-PE dose and longer duration of instillation promotes BTX-A delivery across the urothelium.

Interpreting our urodynamic data has to be done with caution because none of the differences in ICI over time between groups were statistically significant. This may be a reflection of our sample size or the lack of a true effect. Another perplexing finding of 
our study was seen in rats treated with Alexa Fluor ${ }^{\circledR} 594-$ labeled BTX-A 10 U- HA-PE $0.5 \mathrm{~g}$ for 60 minutes instillation. In this group the ICI declined suggesting a worsening from a baseline interval of 16.0 milliseconds to an interval of 11.3 milliseconds after acetic acid instillation. One possible explanation is the potential loss of biological activity resulting from labeling of BTX-A with Alexa Fluor $\AA_{594}$. We could have examined SNAP-5 staining in this group to detect if translocated BTX-A is functionally active or not but this was not done in this study. Clearly this must be investigated further to verify this result and seek potential explanations for this observation.

Our study adds to the body of literature studying alternative methods of BTX-A delivery as opposed to direct detrusor muscle injection (80-84). Chaung et al tested intravesical instillation of BTX-A with liposomes (lipotoxin) in Sprague Dawley rats using similar outcome measures. These results matched ours as we found that mean SNAP-25 staining percentage in group 5A rats treated with high HA-PE dose showed similar lowered mean SNAP-25 staining percentage compared to control groups. In their study, Chaung et al showed a significant decrease in SNAP-25 activity in the lipotoxin pre-treated group which also exhibited less bladder hyperactivity and inflammation in response to acetic acid intravesical instillation. Their report was however lacking some basic data to interpret the results like the number of rats in each group and the lack of untreated controls. Pre-treatment with low-energy shock waves (LESW) was used in another experimental study on female Sprague Dawley rats, in which the effects of intravesical instillation of BTX-A-saline solution were compared after pre-treatment with LESW.

The evaluation included an urodynamic evaluation eight days' post-treatment, as well as staining for SNAP-23, SNAP-25 and COX2 (83). The study showed that SNAP-23, SNAP-25 and COX2 protein levels in the LESW+ BTX-A group was decreased by $15 \%$, $15 \%$ and $28 \%$, respectively compared to the control group.

The results were encouraging, the author mentioned that SNAP-23, SNAP-25 and COX2 protein level in the LESW+ BTX-A group was decreased by $15 \%(\mathrm{p}<0.05), 15 \%$ $(\mathrm{p}=0.061)$ and $28 \%(\mathrm{p}<0.05)$, respectively, compared to the control group. However, no significant difference was detectable in LESW or BTX-A groups relative to controls. Pre- 
treatment with LESW decreased SNAP-25, SNAP-23 and COX2 expression in LESW pre-treated versus non-pre-treated rats, and inhibited bladder hyperactivity induced by an acetic acid challenge, suggesting at least some degree of enhanced urothelial permeability to BTX-A post LESW treatment.

\section{Current study limitations}

Our study has limitations that need to be considered while interpreting its results. The original intention was to perform urodynamic assessment while the rat was awake, instead of under general anesthesia. However, because rats will not lie still even during a relatively innocuous procedure like bladder catheterization, anaesthesia was required. Performing urodynamic assessments while the rats were awake would likely have yielded a more realistic evaluation of bladder function. In addition, group 6 rats were all excluded from the urodynamic analysis because of an issue related to urodynamic procedure and instillation technique leading to leakage and inadequate bladder filling. This was unfortunate but a true learning process given the absence of any previously published guidelines on how to perform urodynamic assessments on an anaesthetised rat. This loss of data from this group prohibited us from determining if 60 minutes versus 30 minutes' bladder instillation actually impacted successful BTX-A delivery. Urodynamic data in this study was obtained rigorously and using a standardized protocol. Despite this, urodynamic data show variability and we are concerned with the validity of this data and the lack of previous study references to justify our observations.

ICI measurements were not universally analyzable because of some studies with either single bladder contraction during the study or flat curve as a result of failure of detrusor contraction under anesthesia. Therefore, it was hard to interpret whether this was a relaxed non-contractile bladder or somehow a flaw in pressure recording or the effect of anaesthesia, though all rat underwent urodynamic studies under anaesthesia.

Future studies expanding on our findings will have to investigate using maximal doses of HA-PE (0.5 g or more) with higher instillation time, beyond 60 minutes to understand the implications of both modifications. This study opened up the possibility of a new novel delivery mechanism that has never been tested before, which does show promise and requires further evidence and experimental study before clinical translation. Lack of blinding of assessors when analyzing SNAP-25 staining would be a pre-requisite for 
future studies but unfortunately could not be implemented in this current study. However, by randomly taking 4 pictures of each slide, then select the picture with the highest SNAP-25 staining percentage, we tried our best to avoid investigator bias.

We had a relatively small sample size especially when we take into account sub group divisions. Future studies using this technique will have the benefit of considering a formal sample size calculation. In addition, selecting a larger animal model with thicker bladder wall will help us estimate the depth of BTX-A penetration using the HA-PE carrier.

\section{Implications of the Research}

This study is a first step to evaluate the success of a novel method to deliver BTX-A to the detrusor muscle using HA-PE. This technique and carrier, if validated in subsequent studies, may eventually preclude the need for endoscopic injection of BTX-A in the detrusor muscle under anesthesia in pediatric patients. The implications for patient comfort, lack of physician led intervention and savings related to health care costs could potentially be very significant if this technique achieves similar results to direct bladder muscle injection.

\section{Conclusions:}

Our study results showed that HA-PE could potentially be an efficient carrier for BTX-A delivery across the urothelium using simple bladder instillation. Further investigation of this potential is required in a larger animal model with a larger sample size and more robust urodynamic data. In addition, the effect of HA-PE dose and instillation time needs to be further elucidated to understand the dose-dependent effect, while ensuring that adverse effects of HA-PE continue to be absent. 


\section{Bibliography}

1) Abrams P, Cardozo L, Fall M, Griffiths D, Rosier P, Ulmsten U, van

Kerrebroeck P, Victor A, Wein A. The standardisation of terminology of lower urinary tract function: report from the Standardisation Sub-committee of the International Continence Society. American journal of obstetrics and gynecology. 2002 Jul 31; 187(1):116-26.

2) Onukwugha E, Zuckerman IH, McNally D, Coyne KS, Vats V, Mullins CD. The total economic burden of overactive bladder in the United States: a diseasespecific approach. The American journal of managed care. 2009 Mar; 15(4 Suppl):S90-7.

3) Stewart W, Van Rooyen J, Cundiff G, Abrams P, Herzog A, Corey R, Hunt T, Wein A. Prevalence and burden of overactive bladder in the United States. World journal of urology. 2003 May 1; 20(6):327-36.

4) Irwin DE, Milsom I, Hunskaar S, Reilly K, Kopp Z, Herschorn S, Coyne K, Kelleher C, Hampel C, Artibani W, Abrams P. Population-based survey of urinary incontinence, overactive bladder, and other lower urinary tract symptoms in five countries: results of the EPIC study. European urology. 2006 Dec 31; 50(6):1306-15.

5) Milsom I, Abrams P, Cardozo L, Roberts RG, Thüroff J, Wein AJ. How widespread are the symptoms of an overactive bladder and how are they managed? A population-based prevalence study. BJU international. 2001 Jun 1; 87(9):760-6.

6) Hanchanale VS, Rao AR, Martin FL, Matanhelia SS. The unusual history and the urological applications of botulinum neurotoxin. Urologia internationalis. 2010 Jul 27; 85(2):125-30.

7) Erbguth FJ, Naumann M. Historical aspects of botulinum toxin Justinus Kerner (1786-1862) and the "sausage poison". Neurology. 1999 Nov 1; 53(8):1850-.

8) Ermengem EV. Ueber einen neuen anaeroben Bacillus und seine Beziehungen zum Botulismus. Medical Microbiology and Immunology. 1897 Feb 10; 26(1):156. 
9) Montecucco C, Schiavo G. Structure and function of tetanus and botulinum neurotoxins. Quarterly reviews of biophysics. 1995 Nov 1; 28(04):423-72.

10) Chapple C, Patel A. Botulinum toxin-new mechanisms, new therapeutic directions? European urology. 2006 Apr 1; 49(4):606-8.

11) Schulte-Baukloh H, Michael T, Schobert J, Stolze T, Knispel HH. Efficacy of botulinum-a toxin in children with detrusor hyperreflexia due to myelomeningocele: preliminary results. Urology. 2002 Mar 31; 59(3):325-7.

12) Mangera A, Andersson KE, Apostolidis A, Chapple C, Dasgupta P, Giannantoni A, Gravas S, Madersbacher S. Contemporary management of lower urinary tract disease with botulinum toxin A: a systematic review of botox (onabotulinumtoxinA) and dysport (abobotulinumtoxinA). European urology. 2011 Oct $31 ; 60(4): 784-95$.

13) Gamé X, Mouracade P, Chartier-Kastler E, Viehweger E, Moog R, Amarenco G, Denys P, De Seze M, Haab F, Karsenty G, Kerdraon J. Botulinum toxin-A (Botox ${ }^{\circledR}$ ) intradetrusor injections in children with neurogenic detrusor overactivity/neurogenic overactive bladder: A systematic literature review. Journal of pediatric urology. 2009 Jun 30; 5(3):156-64.

14) Karsenty G, Denys P, Amarenco G, De Seze M, Gamé X, Haab F, Kerdraon J, Perrouin-Verbe B, Ruffion A, Saussine C, Soler JM. Botulinum toxin A $\left(\right.$ Botox $\left.{ }^{\circledR}\right)$ intradetrusor injections in adults with neurogenic detrusor overactivity/neurogenic overactive bladder: a systematic literature review. european urology. 2008 Feb 29; 53(2):275-87.

15) Chuang YC, Tyagi P, Huang CC, Yoshimura N, Wu M, Kaufman J, Chancellor MB. Urodynamic and immunohistochemical evaluation of intravesical botulinum toxin A delivery using liposomes. The Journal of urology. 2009 Aug 31; 182(2):786-92.

16) Kajbafzadeh AM, Montaser-Kouhsari L, Ahmadi H, Sotoudeh M. Intravesical electromotive botulinum toxin- A administration: Part I-experimental study. Urology. 2011 Jun 30; 77(6):1460-4. 
17) Montaser-Kouhsari L, Kajbafzadeh AM, Ahmadi H, Sotoudeh M. Intravesical Electromotive Botulinum Toxin-A Administration: an Experimental Study. Journal of Pediatric Urology. 2010 Apr 30; 6: S19-20.

18) Chuang YC, Huang TL, Tyagi P, Huang CC. Urodynamic and immunohistochemical evaluation of intravesical botulinum toxin-A delivery using low energy shock waves. The Journal of urology. 2015 Dec 24.

19) Chuang YC, Yoshimura N, Huang CC, Chiang PH, Chancellor MB. Intravesical botulinum toxin-A administration produces analgesia against acetic acid induced bladder pain responses in rats. The Journal of urology. 2004 Oct 31; 172(4):1529-32.

20) Wight TN, Potter-Perigo S. The extracellular matrix: an active or passive player in fibrosis? American Journal of Physiology-Gastrointestinal and Liver Physiology. 2011 Dec 1; 301(6):G950-5.

21) Murano E, Perin D, Khan R, Bergamin M. Hyaluronan: from biomimetic to industrial business strategy. Natural product communications. 2011 Apr; 6(4):555-72.

22) Evanko SP, Tammi MI, Tammi RH, Wight TN. Hyaluronan-dependent pericellular matrix. Advanced drug delivery reviews. 2007 Nov 10; 59(13):135165.

23) Carol A, Drazba JA. Viewing Hyaluronan Imaging Contributes to Imagining New Roles for This Amazing Matrix Polymer. Journal of Histochemistry \& Cytochemistry. 2011 Mar 1; 59(3):252-7.

24) Brown TJ, Alcorn D, Fraser JR. Absorption of hyaluronan applied to the surface of intact skin. Journal of investigative dermatology. 1999 Nov 30; 113(5):740-6.

25) Kaya G, Tran C, Sorg O, Hotz R, Grand D, Carraux P, Didierjean L, Stamenkovic I, Saurat JH. Hyaluronate fragments reverse skin atrophy by a CD44-dependent mechanism. PLoS Med. 2006 Dec 19; 3(12): 493.

26) Symonette CJ, Kaur Mann A, Tan XC, Tolg C, Ma J, Perera F, Yazdani A, Turley EA. Hyaluronan-phosphatidylethanolamine polymers form pericellular coats on keratinocytes and promote basal keratinocyte proliferation. BioMed research international. 2014 Sep 9; 2014. 
27) El-Badawi A, Schenk EA. Dual innervation of the mammalian urinary bladder A histochemical study of the distribution of cholinergic and adrenergic nerves. American Journal of Anatomy. 1966 Nov 1; 119 (3):405-27.

28) Tanagho, 1982. Tanagho EA: The ureterovesical junction: Anatomy and physiology. In: Chishold GD, Williams DI, ed. Scientific Foundation of Urology, Chicago: Year Book; 1982:295-404.

29) Dixon J, Gosling J. Structure and innervation in the human. InThe physiology of the lower urinary tract 1987 (pp. 3-22). Springer London.

30) Zderic SA, Levin RM, Wein AJ. Voiding function and dysfunction: a relevant anatomy, physiology, and pharmacology, and molecular biology. Adult and pediatric urology. 1996; 3:1159-219.

31) Tyagi P, Wu PC, Chancellor M, Yoshimura N, Huang L. Recent advances in intravesical drug/gene delivery. Molecular pharmaceutics. 2006 Aug 7; 3 (4):36979.

32) Lewis SA. Everything you wanted to know about the bladder epithelium but were afraid to ask. American Journal of Physiology-Renal Physiology. 2000 Jun 1; 278 (6): F867-74.

33) Poggi MM, Johnstone PA, Conner RJ. Glycosaminoglycan content of human bladders: a method of analysis using cold-cup biopsies. InUrologic Oncology: Seminars and Original Investigations 2000 Oct 31 (Vol. 5, No. 5, pp. 234-237). Elsevier.

34) Versi E, Cardozo LD, Studd JW, Brincat M, O'dowd TM, Cooper DJ. Internal urinary sphincter in maintenance of female continence. Br Med J (Clin Res Ed). 1986 Jan 18; 292 (6514):166-7.

35) Parsons CL, Boychuk D, Jones S, Hurst R, Callahan H. Bladder surface glycosaminoglycans: an epithelial permeability barrier. The Journal of urology. 1990 Jan; 143 (1):139-42.

36) Melicow MM. The urothelium: a battleground for oncogenesis. The Journal of urology. $1978 \mathrm{Jul} ; 120$ (1):43-7. 
37) Apodaca G. The uroepithelium: not just a passive barrier. Traffic. 2004 Mar 1; 5 (3):117-28.

38) Hanno PM, Fritz RW, Mulholland SG, Wein AJ. Heparin —examination of its antibacterial adsorption properties. Urology. 1981 Sep 30; 18 (3):273-6.

39) Hurst RE. Structure, function, and pathology of proteoglycans and glycosaminoglycan's in the urinary tract. World journal of urology. 1994 Feb 1; 12 (1):3-10.

40) Andersson KE. Antimuscarinics for treatment of overactive bladder. The Lancet Neurology. 2004 Jan 31; 3(1):46-53.

41) Hegde SS, Choppin A, Bonhaus D, Briaud S, Loeb M, Moy TM, Loury D, Eglen RM. Functional role of M2 and M3 muscarinic receptors in the urinary bladder of rats in vitro and in vivo. British journal of pharmacology. 1997 Apr 1; 120 (8):1409-18.

42) Braverman AS, Tallarida RJ, Ruggieri MR. Interaction between muscarinic receptor subtype signal transduction pathways mediating bladder contraction. American Journal of Physiology-Regulatory, Integrative and Comparative Physiology. 2002 Sep 1; 283 (3):R663-8.

43) Bschleipfer T, Schukowski K, Weidner W, Grando SA, Schwantes U, Kummer W, Lips KS. Expression and distribution of cholinergic receptors in the human urothelium. Life sciences. 2007 May 30; 80 (24):2303-7.

44) Chess-Williams R. Muscarinic receptors of the urinary bladder: detrusor, urothelial and pre-junctional. Autonomic and Autacoid Pharmacology. 2002 Jun 1; 22 (3):133-45.

45) Michel MC, Vrydag W. $\alpha 1$-, $\alpha 2$-and $\beta$-adrenoceptors in the urinary bladder, urethra and prostate. British journal of pharmacology. 2006 Feb 1; 147 (S2):S88119.

46) Malloy BJ, PRICE DT, PRICE RR, BIENSTOCK AM, DOLE MK, FUNK BL, RUDNER XL, RICHARDSON CD, DONATUCCI CF, SCHWINN DA. Alpha 1adrenergic receptor subtypes in human detrusor. The Journal of urology. 1998 Sep $30 ; 160$ (3):937-43. 
47) Andersson KE, Arner A. Urinary bladder contraction and relaxation: physiology and pathophysiology. Physiological reviews. 2004 Jul 1; 84 (3):935-86.

48) de Groat WC, Yoshimura N. Mechanisms underlying the recovery of lower urinary tract function following spinal cord injury. Progress in brain research. 2006 Dec 31; 152:59-84.

49) O'REILLY BA, Kosaka AH, Knight GF, Chang TK, Ford AP, Rymer JM, Popert R, Burnstock G, McMAHON SB. P2X receptors and their role in female idiopathic detrusor instability. The Journal of urology. 2002 Jan 31; 167(1):15764.

50) Thor KB, de Groat WC. Neural control of the female urethral and anal rhabdosphincters and pelvic floor muscles. American Journal of PhysiologyRegulatory, Integrative and Comparative Physiology. 2010 Aug 1; 299(2): R41638.

51) de Groat WC, Yoshimura N. Afferent nerve regulation of bladder function in health and disease. In Sensory Nerves 2009 (pp. 91-138). Springer Berlin Heidelberg.

52) Kanai A, Andersson KE. Bladder afferent signaling: recent findings. The Journal of urology. 2010 Apr 30; 183 (4):1288-95.

53) Holstege G. Micturition and the soul. Journal of Comparative Neurology. 2005 Dec 5; 493 (1):15-20.

54) Schneider T, Fetscher C, Krege S, Michel MC. Signal transduction underlying carbachol-induced contraction of human urinary bladder. Journal of Pharmacology and Experimental Therapeutics. 2004 Jun 1; 309 (3):1148-53.

55) Andersson KE, Arner A. Urinary bladder contraction and relaxation: physiology and pathophysiology. Physiological reviews. 2004 Jul 1; 84 (3):935-86.

56) Fry CH, Skennerton D, Wood D, Wu C. The cellular basis of contraction in human detrusor smooth muscle from patients with stable and unstable bladders. Urology. 2002 May 31; 59 (5):3-12. 
57) Braverman AS, Doumanian LR, Ruggieri MR. M2 and M3 muscarinic receptor activation of urinary bladder contractile signal transduction. II. Denervated rat bladder. Journal of Pharmacology and Experimental Therapeutics. 2006 Feb 1; 316 (2):875-80.

58) Mourtzinos A, Stoffel JT. Management goals for the spina bifida neurogenic bladder: a review from infancy to adulthood. Urologic Clinics of North America. 2010 Nov 30;37(4):527-35.

59) Snodgrass WT, Gargollo PC. Urologic care of the neurogenic bladder in children. Urologic Clinics of North America. 2010 May 31; 37 (2):207-14.

60) Schurch B, Stöhrer M, Kramer G, Schmid DM, Gaul G, Hauri D. Botulinum-A toxin for treating detrusor hyperreflexia in spinal cord injured patients: a new alternative to anticholinergic drugs? Preliminary results. The Journal of urology. 2000 Sep 30; 164 (3):692-7.

61) Simpson LL. Identification of the major steps in botulinum toxin action. Annu. Rev. Pharmacol. Toxicol. 2004 Feb 10; 44:167-93.

62) Chancellor MB, Fowler CJ, Apostolidis A, De Groat WC, Smith CP, Somogyi GT, Aoki KR. Drug insight: biological effects of botulinum toxin A in the lower urinary tract. Nature clinical practice Urology. 2008 Jun 1; 5 (6):319-28.

63) Chancellor MB, Fowler CJ, Apostolidis A, De Groat WC, Smith CP, Somogyi GT, Aoki KR. Drug insight: biological effects of botulinum toxin A in the lower urinary tract. Nature clinical practice Urology. 2008 Jun 1; 5 (6):319-28.

64) Dolly JO, Aoki KR. The structure and mode of action of different botulinum toxins. European Journal of Neurology. 2006 Dec 1; 13 (s4):1-9.

65) Breidenbach MA, Brunger AT. New insights into clostridial neurotoxin-SNARE interactions. Trends in molecular medicine. 2005 Aug 31; 11 (8):377-81.

66) Kanai A, Wyndaele JJ, Andersson KE, Fry C, Ikeda Y, Zabbarova I, De Wachter S. Researching bladder afferents - determining the effects of $\beta 3$-adrenergic receptor agonists and botulinum toxin type-A. Neurourology and urodynamics. 2011 Jun 1; 30 (5):684-91. 
67) Ha US, Park EY, Kim JC. Effect of botulinum toxin on expression of nerve growth factor and transient receptor potential vanilloid 1 in urothelium and detrusor muscle of rats with bladder outlet obstruction-induced detrusor overactivity. Urology. 2011 Sep 30; 78 (3):721-e1.

68) Wiseman OJ, Fowler CJ, Landon DN. The role of the human bladder lamina propria myofibroblast. BJU international. 2003 Jan 1; 91(1):89-93.

69) Mangera A, Andersson KE, Apostolidis A, Chapple C, Dasgupta P, Giannantoni A, Gravas S, Madersbacher S. Contemporary management of lower urinary tract disease with botulinum toxin A: a systematic review of botox (onabotulinumtoxinA) and dysport (abobotulinumtoxinA). European urology. 2011 Oct 31; 60 (4):784-95.

70) Gamé X, Mouracade P, Chartier-Kastler E, Viehweger E, Moog R, Amarenco G, Denys P, De Seze M, Haab F, Karsenty G, Kerdraon J. Botulinum toxin-A (Botox ${ }^{\circledR}$ ) intradetrusor injections in children with neurogenic detrusor overactivity/neurogenic overactive bladder: A systematic literature review. Journal of pediatric urology. 2009 Jun 30; 5 (3):156-64.

71) Karsenty G, Denys P, Amarenco G, De Seze M, Gamé X, Haab F, Kerdraon J, Perrouin-Verbe B, Ruffion A, Saussine C, Soler JM. Botulinum toxin A (Botox ${ }^{\circledR}$ ) intradetrusor injections in adults with neurogenic detrusor overactivity/neurogenic overactive bladder: a systematic literature review. european urology. 2008 Feb 29; 53 (2):275-87.

72) Boy S, Schmid M, Reitz A, Von Hessling A, Hodler J, Schurch B. Botulinum toxin injections into the bladder wall-a morphological evaluation of the injection technique using magnetic resonance imaging. European Urology Supplements. 2006 Apr 30; 5 (2):299.

73) Kuo HC. Urodynamic evidence of effectiveness of botulinum A toxin injection in treatment of detrusor overactivity refractory to anticholinergic agents. Urology. 2004 May 31; 63 (5):868-72.

74) Coelho A, Cruz F, Cruz CD, Avelino A. Spread of onabotulinumtoxinA after bladder injection. Experimental study using the distribution of cleaved SNAP-25 as the marker of the toxin action. European urology. 2012 Jun 30; 61 (6):1178-84. 
75) Krhut J, Zvara P. Intravesical instillation of botulinum toxin A: an in vivo murine study and pilot clinical trial. International urology and nephrology. 2011 Jun 1; 43(2):337-43.

76) Petrou SP, Parker AS, Crook JE, Rogers A, Metz-Kudashick D, Thiel DD. Botulinum a toxin/dimethyl sulfoxide bladder instillations for women with refractory idiopathic detrusor overactivity: a phase 1/2 study. InMayo Clinic Proceedings 2009 Aug 31 (Vol. 84, No. 8, pp. 702-706). Elsevier.

77) Munoz A, Somogyi GT, Boone TB, Smith CP. Central inhibitory effect of intravesically applied botulinum toxin A in chronic spinal cord injury. Neurourology and urodynamics. 2011 Sep 1; 30 (7):1376-81.

78) Chuang YC, Huang TL, Tyagi P, Huang CC. Urodynamic and immunohistochemical evaluation of intravesical botulinum toxin A delivery using low energy shock waves. The Journal of urology. 2015 Dec 24.

79) Heinen F, Molenaers G, Fairhurst C, Carr LJ, Desloovere K, Valayer EC, Morel E, Papavassiliou AS, Tedroff K, Pascual-Pascual SI, Bernert G. European consensus table 2006 on botulinum toxin for children with cerebral palsy. European Journal of Paediatric Neurology. 2006 Nov 30; 10 (5):215-25.

80) Chuang YC, Tyagi P, Huang CC, Yoshimura N, Wu M, Kaufman J, Chancellor MB. Urodynamic and immunohistochemical evaluation of intravesical botulinum toxin A delivery using liposomes. The Journal of urology. 2009 Aug 31; 182(2):786-92.

81) Kajbafzadeh AM, Montaser-Kouhsari L, Ahmadi H, Sotoudeh M. Intravesical electromotive botulinum toxin type A administration: Part I—experimental study. Urology. 2011 Jun 30; 77(6):1460-4.

82) Montaser-Kouhsari L, Kajbafzadeh AM, Ahmadi H, Sotoudeh M. Intravesical Electromotive Botulinum Toxin-A Administration: an Experimental Study. Journal of Pediatric Urology. 2010 Apr 30; 6: S19-20.

83) Chuang YC, Huang TL, Tyagi P, Huang CC. Urodynamic and immunohistochemical evaluation of intravesical botulinum toxin A delivery using low energy shock waves. The Journal of urology. 2015 Dec 24. 
84) Chuang YC, Yoshimura N, Huang CC, Chiang PH, Chancellor MB. Intravesical botulinum toxin a administration produces analgesia against acetic acid induced bladder pain responses in rats. The Journal of urology. 2004 Oct 31; 172(4):152932.

85) Parsons CL, Boychuk D, Jones S, Hurst R, Callahan H. Bladder surface glycosaminoglycan's: an epithelial permeability barrier. The Journal of urology. 1990 Jan; 143(1):139-42.

86) Petrou SP, Parker AS, Crook JE, Rogers A, Metz-Kudashick D, Thiel DD. Botulinum a toxin/dimethyl sulfoxide bladder instillation for women with refractory idiopathic detrusor overactivity: a phase 1/2 study. InMayo Clinic Proceedings 2009 Aug 31 (Vol. 84, No. 8, pp. 702-706). Elsevier.

87) Ballard PL, Gonzales LW, Godinez RI, Godinez MH, Savani RC, McCurnin DC, Gibson LL, Yoder BA, Kerecman JD, Grubb PH, Shaul PW. Surfactant composition and function in a primate model of infant chronic lung disease: effects of inhaled nitric oxide. Pediatric research. 2006 Jan 1; 59 (1):157-62.

88) Breitkreutz D, Koxholt I, Thiemann K, Nischt R. Skin basement membrane: the foundation of epidermal integrity - BM functions and diverse roles of bridging molecules nidogen and perlecan. BioMed research international. 2013 Mar 21; 2013.

89) Khan F, Ahmad SR. Polysaccharides and their derivatives for versatile tissue engineering application. Macromolecular bioscience. 2013 Apr 1; 13 (4):395-421.

90) Akram Al-Motabagani M. Age-related changes in the urinary bladder of the female albino rats. International Journal of Morphology. 2005; 23 (4):309-16.

91) Panchuk-Voloshina N; Haugland RP; Bishop-Stewart J; et al. "Alexa dyes, a series of new fluorescent dyes that yield exceptionally bright, photostable conjugates". J. Histochem. Cytochem. 47 (9): 1179-88. Sep1999. 


\section{Appendices}

\section{Appendix 1: SNAP-25 Staining protocol}

1) Tissue slides were de-paraffinized by being immersed in xylene for 15 minutes, followed by alcohol concentrations (100\%,95\% and then $70 \%)$, each for 10 minutes.

2) The tissue was then rehydrated by being immersed in distilled water and then $1 *$ PBS (phosphate-buffered saline), each for 5 minutes.

3) Antigens were retrieved by immersing tissue slides in $10 \mathrm{mM}$ sodium citrate, $\mathrm{pH}$ 6.0, then micro-waving them at power level 10 for three minutes, followed by power level 5 for five minutes and, finally, power level 3 for eight minutes. Slides then were left to cool for 20-30 minutes at room temperature.

4) Slides were washed in 1 *PBS for 5 minutes, a process that was repeated 2-3 times.

5) Slides were immersed in 3.0\% $\mathrm{H} 2 \mathrm{O} 2$ diluted in 1 *PBS for 10 minutes.

6) Slides were removed from $\mathrm{H} 2 \mathrm{O} 2$ and washed in 1 *PBS for five minutes, and the washing process was repeated 2-3 times.

7) Blocking with 3.0\% BSA (Bovine Serum Albumin) diluted in 1 *PBS was done for one to two hours, after which one slide was retained as a negative control in BSA solution in a $4^{\circ} \mathrm{C}$ fridge overnight.

8) Primary antibody (sab 2500965, Sigma Aldrich, Canada) was added to the slides at a dilution of 1:100ul (SNAP-25 antibodies were supplied at $0.5 \mathrm{mg} / \mathrm{mL}$ in saline with $0.02 \%$ sodium azide and $0.5 \%$ bovine serum albumin). Then the slides were covered in tinfoil, and stored in a $4^{\circ} \mathrm{C}$ fridge overnight. 


\section{Day 2:}

1) The negative 'control' slide was taken out of the fridge and washed with the other slides in 1 PBS for five minutes on the rocker, and this repeated 2-3 times.

2) Secondary antibodies (anti-rabbit IgG-goat), at a dilution of $1 \mu 1$ in $500 \mu l$ of 1 *PBS, were added to the slides and this was left for two hours at room temperature, followed by washing in $1 * \mathrm{PBS}$ for 15 minutes.

3) Streptavidin was added to the slides at a dilution of 1:2000 $\mu 11 * \mathrm{PBS}$ and left at room temperature for 30 minutes. Then the slides were washed in $1 * \mathrm{PBS}$ for five minutes.

4) $\mathrm{DAB}$ was added and the slides left to sit for between 5 and 40 minutes, the length of time determined by evidence of a reaction (Brown staining). As soon as slides started to turn brown, they were washed in 1 *PBS for five minutes, and this repeated 2-3 times.

5) Sections were immersed in filtered haematoxylin for 30 seconds, then washed in distilled water for five minutes.

6) Tissue slides were then run through the original series in a reverse concentration of ethanol ( $70 \%-95 \%-100 \%$ ), each for 2 minutes, culminating with xylene for one minute.

7) As a final step, the slides were mounted in Richard-Allan Scientific Cytoseal 60 (Thermo-Scientific) and left to dry overnight in the laboratory. 


\section{Appendix 2: BTX-A- Alexa Fluor®594 labeling Protocol}

\section{Solution preparation:}

The first step in solution preparation was to make a sodium bicarbonate solution with a $\mathrm{pH}$ of 9.0 , by dissolving $1.2 \mathrm{~g} / \mathrm{L}$ of sodium bicarbonate in distilled water and adjusting the $\mathrm{pH}$ with $1 \mathrm{~N} \mathrm{NaOH}$ to achieve the final desired $\mathrm{pH}$ of 9.0. The Alexa solution was then prepared by dissolving $1 \mathrm{mg}$ Alexa Fluor ${ }^{\circledR 594}$ in $250 \mu \mathrm{L}$ DMSO, which then was added to $250 \mathrm{~mL}$ of $0.1 \mathrm{M}$ sodium bicarbonate, at $\mathrm{pH}=9.0$. The dialysis solution for BTX-A was $4 \mathrm{~L}$ of $1 *$ PBS (phosphate buffered saline).

\section{Steps:}

Solutions were prepared as mentioned above and stored at $4{ }^{\circ} \mathrm{C}$ for 24 hours, after which a length of dialysis tubing was soaked in RT-PCR grade water for 2-5 minutes, then in boiling water for 5 minutes, followed by final incubation, again in RT-PCR grade water. This was followed by re-suspension of the BTX-A in a $0.1 \mathrm{M}$ solution of sodium bicarbonate at $\mathrm{pH}=9.0$.

BTX-A was placed into dialysis tubing and dialyzed in $0.1 \mathrm{M}$ sodium bicarbonate, $\mathrm{pH} 9.0$, at $4^{\circ} \mathrm{C}$ with $250 \mathrm{uL}$ of dye in $250 \mathrm{~mL}$ of $0.1 \mathrm{M}$ sodium bicarbonate $\mathrm{pH} 9.0$, then left for 24 hours at $4^{\circ} \mathrm{C}$ in the dark (wrapped in foil). Finally, BTX-A was dialyzed in $1 * \mathrm{PBS}$ for 2-3 days, while changing the buffer every 6-12 hours (6 changes). 


\section{Appendix 3: Animal Use Approval}

2012-047:3:

AUP Number: 2012-047

AUP Title: Use of hyaluronan formulations in tissues

Yearly Renewal Date: 05/01/2016

\section{The YEARLY RENEWAL to Animal Use Protocol (AUP) 2012-047 has been approved, and will be approved for one year following the above review date.}

1. This AUP number must be indicated when ordering animals for this project.

2. Animals for other projects may not be ordered under this AUP number.

3. Purchases of animals other than through this system must be cleared through the ACVS office.

Health certificates will be required.

\section{REQUIREMENTS/COMMENTS}

Please ensure that individual(s) performing procedures on live animals, as described in this protocol, are familiar with the contents of this document.

The holder of this Animal Use Protocol is responsible to ensure that all associated safety components (biosafety, radiation safety, general laboratory safety) comply with institutional safety standards and have received all necessary approvals. Please consult directly with your institutional safety officers.

Submitted by: Kinchlea, Will D

on behalf of the Animal Use Subcommittee

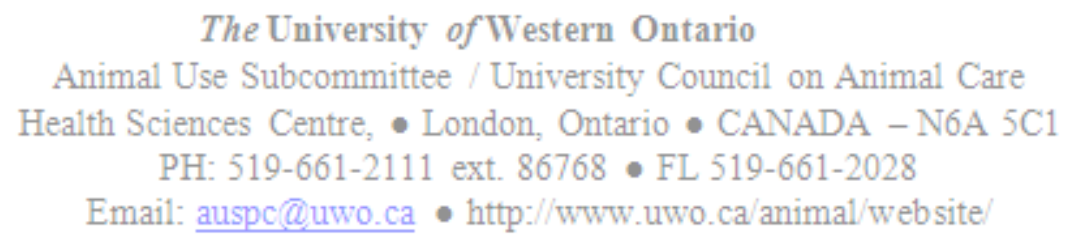




\section{Appendix 4: Urodynamic Assessment}

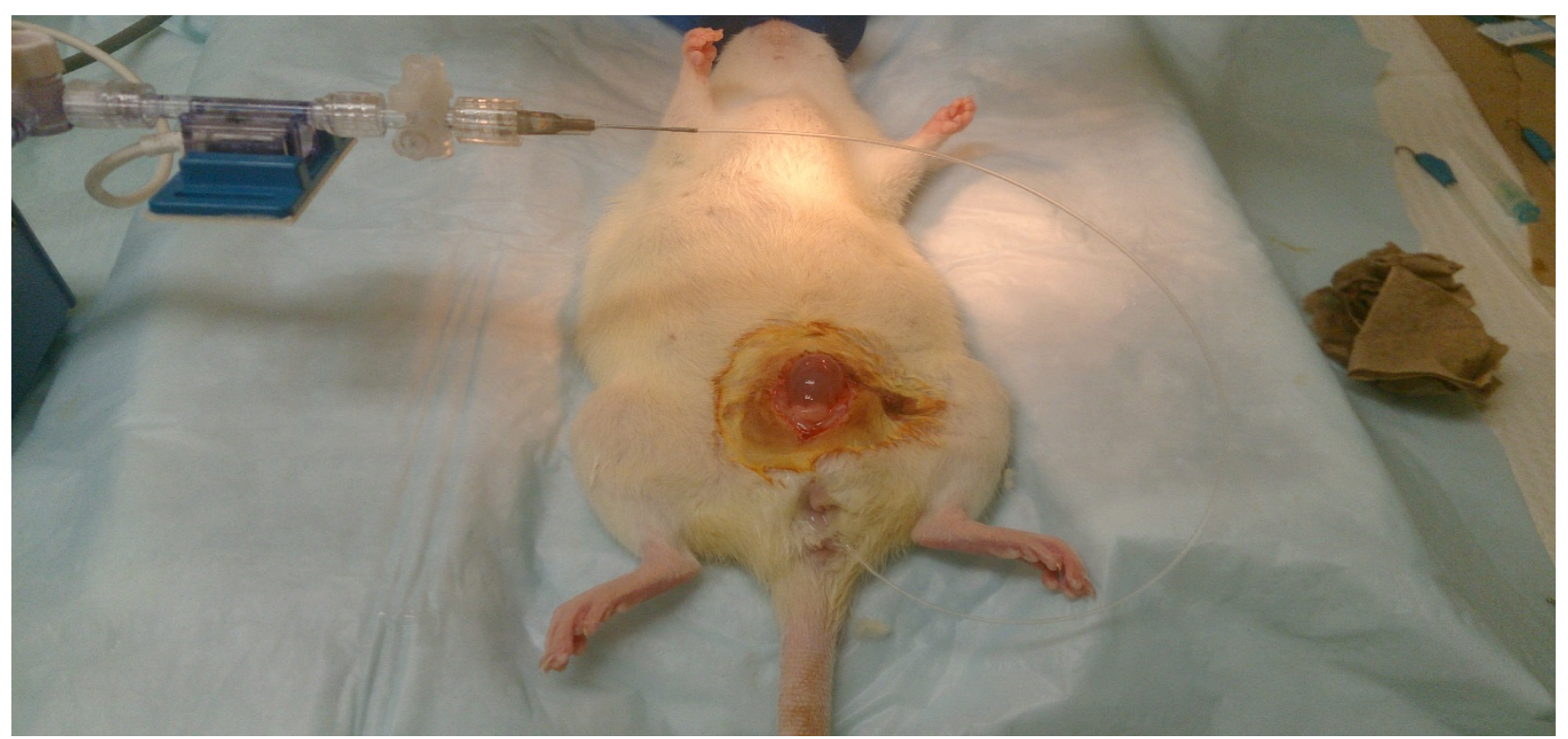

Urodynamic assessment via PE-50 urethral tube

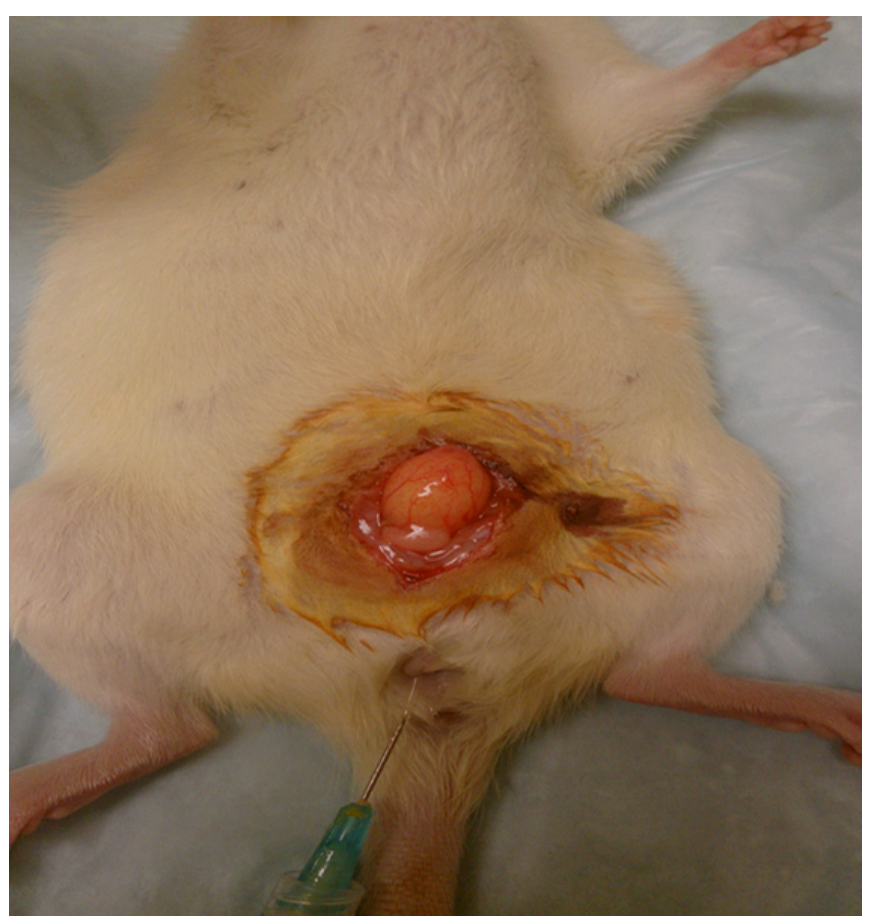

BTX-A HA-PE saline bladder instillation via PE-50 urethral tube 


\section{Curriculum Vitae}

Name: Mohamed El Shatoury

Post-secondary M.B.B.Ch. Cairo University,

Education and Cairo, Egypt, 1996

Degrees:

Master's of Urology, Cairo University, Cairo, Egypt, 2005

Master's of science in Surgery

The University of Western Ontario

London, Ontario, Canada, 2016

Honours and Canada Student Grant, May 2015

Awards:

Ontario Student Opportunity Grant, July 2016

CUASF-SIU International Scholarship, June 2016

Related Work Urology Assistant Consultant, Department of Surgery, Division of Urology,

Experience: King Abdul Aziz Medical city, Riyadh -Saudi Arabia.

Urology Assistant Consultant, Omaru Shehu Hospital, Nigeria.

Urology Assistant consultant, Damietta Specialized Hospital, Egyptian Ministry of Health, Egypt.

Urology Resident, Cairo University Hospitals, Cairo, Egypt, 7/2000 to

$5 / 2005$.

\section{Publications:}

- El Shatoury M, De Young L, Dave S; A novel delivery mechanism of Botulinum Toxin/A a cross the the urothelium using a newly modulated carrier to replace conventional detrusor injection (In Progress).

- Dave S, El Shatoury M, Peter Wang; A Pilot Randomized Controlled Trial Comparing Botulinum Toxin A versus Oxybutynin Therapy in the Spina Bifida Associated Neurogenic Bladder (In Progress) 